\title{
High order direct parametrisation of invariant manifolds for model order reduction of finite element structures: application to generic forcing terms and parametrically excited systems
}

Andrea Opreni ( $\sim$ andrea.opreni@polimi.it)

Politecnico di Milano https://orcid.org/0000-0003-1397-6435

Alessandra Vizzaccaro

University of Bristol

Cyril Touzé

ENSTA ParisTech: Ecole Nationale Superieure de Techniques Avancees

Attilio Frangi

Politecnico di Milano

\section{Research Article}

Keywords: finite element method, geometric nonlinearities, model order reduction, invariant manifold, whisker, non-autonomous systems

Posted Date: February 21st, 2022

DOI: https://doi.org/10.21203/rs.3.rs-1359763/v1

License: (a) (i) This work is licensed under a Creative Commons Attribution 4.0 International License. Read Full License 


\title{
High order direct parametrisation of invariant manifolds for model order reduction of finite element structures: application to generic forcing terms and parametrically excited systems
}

\author{
Andrea Opreni $^{1}$ • Alessandra Vizzaccaro ${ }^{2,3} \cdot$ Cyril \\ Touzé $^{4}$. Attilio Frangi ${ }^{1}$
}

the date of receipt and acceptance should be inserted later

\begin{abstract}
The direct parametrisation method for invariant manifolds is used for model order reduction of forced-damped mechanical structures subjected to geometric nonlinearities. Nonlinear mappings are introduced, allowing one to pass from the degrees of freedom of the finite-element model to the normal coordinates. Arbitrary order of expansions are considered for the unknown mappings and the reduced dynamics, which are then solved sequentially through the homological equations, for both autonomous and time-dependent terms. It is emphasized that the two problems share a similar structure, which can be used for an efficient implementation of the non-autonomous added terms. A special emphasis is also put on the new resonance conditions arising due to the presence of the external forcing frequencies, which allows predicting phenomena such as parametric excitation and isolas formation. The method is then applied to structures of academic and industrial interest. First, the large amplitude vibrations of a forceddamped cantilever beam are studied. This example highlights that high order non-autonomous terms are compulsory to correctly estimate the maximum vibration amplitude experienced by the structure. The birth of isolated solutions is also illustrated on this example. The cantilever is then used to show how quadratic coupling creates conditions for the excitation of the parametric instability, all these features being correctly embedded in the reduction process. A shallow arch excited with multi-modal forcing is then studied to detail different forcing effects. Finally, the approach is validated on a structure of industrial relevance, i.e. a comb driven micro-electro-mechanical resonator. The accuracy and computational performance reported suggest that the proposed methodology can accurately predict the nonlinear dynamic response of a large class of nonlinear vibratory systems.
\end{abstract}

Keywords finite element method · geometric nonlinearities · model order reduction · invariant manifold · whisker $\cdot$ non-autonomous systems

\section{Introduction}

This work is concerned with model order reduction techniques based on nonlinear transformations for forceddamped vibratory systems subjected to geometric nonlinearities, with emphasis on large dimensional finite element (FE) models stemming from the discretisation of the governing partial differential equation. A major motivation is the need of efficient reduction techniques in the semiconductor industry, where the

\footnotetext{
1 Department of Civil and Environmental Engineering

Politecnico di Milano

Piazza Leonardo da Vinci, 32, 20133 Milano MI

Tel.: +39.349.377.6864

E-mail: andrea.opreni@polimi.it

2 Department of Engineering Mathematics

University of Bristol

Bristol BS8 1UB

3 Department of Mechanical Engineering

Imperial College London

Exhibition Rd, South Kensington, London SW7 2BX
}

\footnotetext{
${ }^{4}$ Institute of Mechanical Sciences and Industrial Applications (IMSIA)

ENSTA Paris - CNRS - EDF - CEA - Institut Polytechnique de Paris

828 boulevard des maréchaux 91762 Palaiseau cedex
} 
design stage is of primary importance and necessitates a fast and agile prediction of the steady dynamic response of complex structures [1. Furthermore, the development of systems operating in a fully nonlinear regime poses tight design requirements for proposing solutions based on features such as mode interaction [2] or parametric excitation [3].

Reduction methods for geometrically nonlinear structures have now a long history, and numerous approaches have been proposed that can be roughly divided into linear and nonlinear techniques 4,5]. Linear methods, like modal projection, rely on a linear change of coordinates and have been used for decades. However, in presence of nonlinearities, the dimension of the subspace required to define a proper embedding for system solutions increases importantly, hence making linear projections techniques less efficient for dimensionality reduction 6, 7,8, This is a direct consequence of the loss of invariance of linear subspaces, like modal subspaces or POD bases 9,7, and has stimulated an interest towards the invariant manifold theory [10,11,12,13, for dynamical systems.

Along this direction, an important research effort has thus been devoted to the definition of nonlinear normal modes (NNMs) in order to extend the properties of the linear modal basis to the nonlinear regime, mainly by preserving the invariance property [10,11,14. For conservative vibratory systems, where the eigenspectrum is composed of pairs of purely imaginary complex conjugates, the center theorem from Lyapunov [15,16] guarantees the existence of two-dimensional manifolds densely filled with periodic orbits, named Lyapunov subcenter manifolds (LSM), thus offering a solid framework to define an NNM as a family of periodic orbits lying on the LSM attached to the selected pair of conjugate eigenvalues. In the dissipative case, Shaw and Pierre proposed to define an NNM as an invariant manifold that is tangent to its linear counterpart at origin [10]. As remarked by numerous investigators [17, 18, this definition however lacks unicity since the phase space is foliated with an infinity of invariant manifolds tangent at the origin to a linear modal subspace. To retrieve uniqueness, Haller and Ponsioen showed that among all these invariant subsets, there exist a unique two-dimensional manifold with the highest continuity degree, under non-resonance conditions and for expansion orders exceeding the so-called spectral quotient [18. They then defined this unique invariant manifold as the spectral submanifold (SSM) and used the SSM for the purpose of model order reduction [18, 19. As commented in [5, following the original ideas and definitions of Shaw and Pierre, this unique SSM is thus the NNM that was computed with low-order approximations in earlier studies 20,21,22. Importantly, uniqueness is retrieved only once the order of the expansion reaches the spectral quotient, which in practical applications is generally a very large integer, so that all proposed computations generally lead only to an approximation of the SSM. Application of these methods based on the invariant manifold theory has enabled meaningful results in model order reduction of geometrically nonlinear structures [23, 20,21, 13, 24].

Recently, an important step forward has been realised in the mathematical community thanks to the introduction of the parametrisation method for invariant manifolds (PIM), first formalized by Cabré, Fontich, and de la Llave with the purpose of deriving the invariant manifolds attached to fixed points in maps 25], 26. 27. Starting from the invariance equation [28, the procedure allows unifying in the same framework [5] previous approaches that used either the center manifold theorem [10] or the normal form approach [13. The PIM has been first introduced in the nonlinear vibration community in a series of papers by Haller and coworkers [18, 19,29,30,31. An important improvement, as compared to previous developments that only considered low-order approximations, has also been to introduce an automatic procedure allowing to reach arbitrary orders in the expansions, ensuring convergence of the reduced order models [29].

However, the PIM was originally formulated for systems expressed in modal coordinates, thus requiring the computation of the complete set of eigenfunctions as a first step. This in turn represented a major bottleneck for the application of the method to real structures. In the field of vibration theory, the problem has been recently overcome by proposing direct techniques that can be applied in the physical space and hence directly to the nodal degrees of freedom of a FE mesh. Elaborating on the normal form approach discussed in [13 22, a direct normal form (DNF) has been proposed in 32, then reformulated for generalization purpose in 33 and applied to MEMS (Micro Electro Mechanical Systems) structures. At the same time and elaborating on the parametrisation method, a direct formulation with extension to arbitrary order of expansions in the asymptotic development has been proposed in [34,35], and applied to problems with internal resonances in 36 , 37.

The direct parametrisation method for invariant manifolds (DPIM), as it is proposed for example in 35, has been formulated in the context of autonomous systems, such that the presence of the external forcing was introduced approximately by considering only the global translation of the invariant manifold created by the presence of harmonic forcing, while neglecting its deformations. When external forcing comes into play, the picture is indeed different and may lead to computing a manifold for each forcing frequency, as first observed by 38. Time-dependent manifolds are taken into account in 34 where, however, only a zero-order truncation of the non-autonomous asymptotic development is considered, thus yielding, in the case of modal forcing, the same approximations as the one used in 35. This approach is not sufficient 
for modeling systems subjected to large forcing values with generic distributions or for systems that are parametrically excited. This class of problems requires a proper treatment of the non-autonomous system.

In the dynamical systems community, the PIM has been extended to take into account non-autonomous terms for maps in a series of contributions 39,40,41. In these papers, time-dependent manifolds are often referred to as whiskers, which generally represent a stable (or unstable) manifold attached to a periodic orbit or an invariant torus. In the remainder of the paper, we will use the wording time-dependent invariant manifolds or whiskers to refer to the computed structures in phase space. Following this general setting, PIM has been applied to vibratory systems in order to enlarge the scope and properly derive ROMs for forced-damped systems in the following papers by Haller et al. [18, 30, 42, 43. More specifically, a zeroorder assumption for the non-autonomous part is retained in 30, the idea being to derive simplified formulas to address the relationship between backbone curves and frequency response curves (FRCs). In 42, general expressions for the non-autonomous part are considered in an appendix, but the main part of the derivation is concerned with proposing an analytical criterion to detect isolated solutions. The most complete derivation is detailed in [43, and the computations relative to both autonomous and non-autonomous part have been made publicly available in the code SSMtool 1.0. However, all these developments used the PIM such that the dynamics in the modal basis needs to be given as starting point. Also, in the numerical examples, the computations of the whiskers are detailed for each external forcing frequency, which constitutes a limitation since ROMs are more likely to be used for parameter continuations as in FRCs, where the continuation is performed with respect to the external frequency. The need to recompute the ROM for each forcing frequency thus constitutes a drawback, already underlined in 38 .

In this contribution, a method is derived allowing to compute ROMs for forced-damped systems. The method leverages the parametrisation method developed in the dynamical systems community [39, 40, 41, and later applied to nonlinear vibration problems in 30,34,36,37, but allows one to use an arbitrary order of expansion for the terms generated by the forcing, and generates ROMs that can be used to produce FRCs with the forcing frequency as continuation parameter. Overcoming this limitation is an important step and comes with two main consequences that can be separately analyzed. On the one hand, the whisker will show some deformations along the phase of the forcing, which are now exactly computed. On the other hand, the reduced dynamics is also modified and new important terms appear, making the link with parametric excitation and isolated solutions. These two effects can be analyzed separately and give rise to different phenomena. Furthermore, we report extensive examples of structures that require such accuracy in the method to correctly predict vibration amplitudes.

The remainder of the work is structured as follows. In Section 2 we report the general set of equations of motion for mechanical systems subjected to geometric nonlinearities. In Section 3 the DPIM in the context of non-autonomous systems is presented in a general framework and its extension to the parametrisation of whiskers is detailed. Explicit expressions for the solution to the homological equations are detailed together with a unified framework for solving both invariance equations. Among the different points discussed in the present Section, we remark that the core of the method is reported in Section 3.1. while the remainder of the Section is devoted to the analytical and numerical aspect of the problem. In Section 4, extensive analysis of the new results provided by the non-autonomous setting are reported. New trivial resonance relationships are highlighted. In Section 5 we detail computational aspects regarding the realification procedure assuming a real-valued forcing. In Section 6, the proposed methodology is applied to large finite element models of structures subjected to loading conditions that require the present approach to correctly predict their nonlinear dynamical response. Finally, in Section 7 we summarise the outcomes of the presented work.

\section{Equations of motion and spectral properties}

\subsection{Continuous problem, FE formulation and forcing representation}

In the present Section the governing equations are derived from a continuum mechanics perspective. Structures are modeled in the framework of large transformations but small strains, and the governing equation is the conservation of linear momentum with proper boundary conditions, as derived in classical textbooks, see e.g. 44]:

$$
\begin{array}{ll}
\rho \ddot{\boldsymbol{u}}-\operatorname{Div} \mathbf{P}=\varepsilon \rho \boldsymbol{b}, & \text { in } \mathrm{B} \times t \in\left(t_{0}, T\right], \\
\mathbf{P} \cdot \boldsymbol{n}=\varepsilon \boldsymbol{t}, & \text { on } \Gamma^{N} \times t \in\left(t_{0}, T\right], \\
\boldsymbol{u}=\overline{\boldsymbol{u}}, & \text { on } \Gamma^{D} \times t \in\left(t_{0}, T\right], \\
\boldsymbol{u}=\boldsymbol{u}_{0}, & \text { in } \mathrm{B} \times t=t_{0}, \\
\dot{\boldsymbol{u}}=\dot{\boldsymbol{u}}_{0}, & \text { in B } \times t=t_{0} .
\end{array}
$$


All quantities are defined in the reference configuration B. $\rho$ is the material density, $\boldsymbol{u}$ the displacement field, $\ddot{(\cdot)}$ stands for double time derivative, $\operatorname{Div}(\cdot)$ is the divergence operator, $\mathbf{P}$ is the first Piola-Kirchhoff stress tensor, $\varepsilon$ is a real-valued book-keeping parameter, and $\boldsymbol{b}$ is the body force field defined per unit mass. $\boldsymbol{n}$ is the unit outward vector defined over the boundary $\Gamma^{N}, \boldsymbol{t}$ is the configuration-independent surface traction vector, $\overline{\boldsymbol{u}}$ is the prescribed displacement along the boundary $\Gamma^{D} \cdot \boldsymbol{u}_{0}$ is the displacement at the initial time $t_{0}$ and $\dot{\boldsymbol{u}}_{0}$ is the initial velocity. We neglect rigid body motions by imposing that $\Gamma^{D}$ is not an empty set.

The book-keeping parameter $\varepsilon$ has been introduced to underline that the external forcing is a priori considered small, in the sense that it shall lead to large amplitude vibrations of the structure with small strains, in order to respect the assumptions of geometric nonlinearity. Note however that in the forthcoming developments $\varepsilon$ is mainly used in order to distinguish, in the analytical derivations, the non-autonomous part from the terms where the forcing is involved. Since each part will be solved with asymptotic expansions of arbitrary order, the assumption of small forcing appears to be little restrictive, and the numerical results reported in Section 6 will underline that the method can handle important forcing values as long as small strains are conserved. In order to assess quantitatively this assumption of small forcing and the criterion to respect, a nondimensional forcing parameter will be introduced and discussed when coming to the numerical examples reported in Section 6. This will provide a valuable quantitative assessment of the small forcing assumption.

Equations (1) represent the well known strong form of the conservation of linear momentum. We here derive a discretisation of the equation by means of a Galerkin approach, hence we recast Eq. (1) in its weak form. To this aim, we introduce the function $\boldsymbol{w}$ defined over the space $\mathcal{C}(\mathbf{0})$ of sufficiently continuous functions vanishing on the portion of the boundary where Dirichlet boundary conditions on the displacement field are imposed. By projecting Eq. (1) on $\boldsymbol{w}$ and integrating over the domain, the classical weak form of the equation governing the conservation of linear momentum, also known as principle of virtual power, is obtained as [44:

$$
\int_{\mathrm{B}} \rho \ddot{\boldsymbol{u}} \cdot \boldsymbol{w} d \mathrm{~B}+\int_{\mathrm{B}} \mathbf{P}: \nabla^{T} \boldsymbol{w} d \mathrm{~B}=\varepsilon\left(\int_{\mathrm{B}} \rho \boldsymbol{b} \cdot \boldsymbol{w} d \mathrm{~B}+\int_{\Gamma^{N}} \boldsymbol{t} \cdot \boldsymbol{w} d \Gamma\right), \quad \forall \boldsymbol{w} \in \mathcal{C}(\mathbf{0}) .
$$

where $\nabla(\cdot)$ denotes the material gradient. Since we are interested in studying vibratory systems subjected to geometric nonlinearity only, a Saint Venant-Kirchhoff constitutive model is assumed, i.e. $\mathbf{S}=\mathcal{A}: \boldsymbol{e}$, where $\mathbf{S}$ is the second Piola-Kirchhoff stress tensor, with $\mathbf{P}=(\mathbf{I}+\nabla \boldsymbol{u}) \cdot \mathbf{S}, \mathcal{A}$ is the elasticity tensor and $\boldsymbol{e}$ is the Green-Lagrange strain tensor. Let us now define the following quantities:

$$
\varepsilon(\boldsymbol{a})=\frac{1}{2}\left(\nabla \boldsymbol{a}+\nabla^{T} \boldsymbol{a}\right), \quad \gamma(\boldsymbol{a}, \boldsymbol{b})=\frac{1}{2}\left(\nabla^{T} \boldsymbol{a} \cdot \nabla \boldsymbol{b}+\nabla^{T} \boldsymbol{b} \cdot \nabla \boldsymbol{a}\right),
$$

where $\boldsymbol{\varepsilon}(\boldsymbol{a})$ is simply the symmetric part of the gradient of a generic vector field $\boldsymbol{a}$ and $\boldsymbol{\gamma}(\boldsymbol{a}, \boldsymbol{b})$ is the symmetric part of the product of the gradients of $\boldsymbol{a}$ and $\boldsymbol{b}$. We remark that $\boldsymbol{\varepsilon}(\boldsymbol{u})$ is the classical small displacement strain measure and that the Green-Lagrange tensor can be expressed as $\boldsymbol{e}=\boldsymbol{\varepsilon}(\mathbf{u})+(1 / 2) \gamma(\mathbf{u}, \mathbf{u})$. By means of these quantities the internal power splits into linear, quadratic and cubic powers of the displacement as:

$$
\begin{aligned}
\int_{\mathrm{B}} \mathbf{P}: \nabla^{T} \boldsymbol{w} d \mathrm{~B}= & \int_{\mathrm{B}} \varepsilon(\boldsymbol{u}): \mathcal{A}: \boldsymbol{\varepsilon}(\boldsymbol{w}) d \mathrm{~B} \\
& +\int_{\mathrm{B}} \frac{1}{2} \gamma(\boldsymbol{u}, \boldsymbol{u}): \mathcal{A}: \boldsymbol{\varepsilon}(\boldsymbol{w})+\boldsymbol{\varepsilon}(\boldsymbol{u}): \mathcal{A}: \gamma(\boldsymbol{u}, \boldsymbol{w}) d \mathrm{~B} \\
& +\int_{\mathrm{B}} \frac{1}{2} \gamma(\boldsymbol{u}, \boldsymbol{u}): \mathcal{A}: \boldsymbol{\gamma}(\boldsymbol{u}, \boldsymbol{w}) d \mathrm{~B} .
\end{aligned}
$$

In the context of the DPIM, splitting nonlinear terms in increasing order is necessary for developing fast and efficient algorithms. Moving to a numerical discretisation, we chose a standard finite dimensional trial space $\mathcal{C}_{h}$. As a consequence, the problem now recasts in finding $\boldsymbol{u}_{h} \in \mathcal{C}_{h}$ such that:

$$
\begin{aligned}
& \int_{\mathrm{B}} \rho \ddot{\boldsymbol{u}}_{h} \cdot \boldsymbol{w}_{h} d \mathrm{~B}+\int_{\mathrm{B}} \boldsymbol{\varepsilon}\left(\boldsymbol{u}_{h}\right): \mathcal{A}: \boldsymbol{\varepsilon}\left(\boldsymbol{w}_{h}\right) d \mathrm{~B} \\
& +\int_{\mathrm{B}} \frac{1}{2} \gamma\left(\boldsymbol{u}_{h}, \boldsymbol{u}_{h}\right): \mathcal{A}: \varepsilon\left(\boldsymbol{w}_{h}\right)+\boldsymbol{\varepsilon}\left(\boldsymbol{u}_{h}\right): \mathcal{A}: \gamma\left(\boldsymbol{u}_{h}, \boldsymbol{w}_{h}\right) d \mathrm{~B} \\
& +\int_{\mathrm{B}} \frac{1}{2} \gamma\left(\boldsymbol{u}_{h}, \boldsymbol{u}_{h}\right): \mathcal{A}: \gamma\left(\boldsymbol{u}_{h}, \boldsymbol{w}_{h}\right) d \mathrm{~B}= \\
& \varepsilon\left(\int_{\mathrm{B}} \rho \boldsymbol{b} \cdot \boldsymbol{w}_{h} d \mathrm{~B}+\int_{\Gamma^{N}} \boldsymbol{t} \cdot \boldsymbol{w}_{h} d \Gamma\right) \quad \forall \boldsymbol{w}_{h} \in \mathcal{C}_{h}(\mathbf{0}) .
\end{aligned}
$$


Where the subscript $(\cdot)_{h}$ denotes fields and spaces approximated in the sense of isoparametric FEs. Upon integration of Eq. (5) for any independent choice of $\boldsymbol{w}_{h}$, the following system of coupled nonlinear differential equations is retrieved:

$$
\mathbf{M} \ddot{\mathbf{U}}+\mathbf{K U}+\boldsymbol{G}(\mathbf{U}, \mathbf{U})+\boldsymbol{H}(\mathbf{U}, \mathbf{U}, \mathbf{U})=\varepsilon \hat{\mathbf{F}} .
$$

where $\mathbf{U}$ is an array collecting all the unknown nodal values for $\mathbf{u}_{h}, \mathbf{M}$ is the mass matrix, $\mathbf{K}$ is the stiffness matrix, $\boldsymbol{G}(\mathbf{U}, \mathbf{U})$ is the quadratic nonlinearity tensor, $\boldsymbol{H}(\mathbf{U}, \mathbf{U}, \mathbf{U})$ is the cubic nonlinearity tensor, and $\hat{\mathbf{F}}$ is the external force vector.

A Rayleigh damping is here assumed, whose continuous and discrete formulations write:

$$
\int_{\mathrm{B}} \alpha \rho \dot{\boldsymbol{u}}_{h} \cdot \boldsymbol{w}+\beta \varepsilon\left(\dot{\boldsymbol{u}}_{h}\right): \mathcal{A}: \boldsymbol{\varepsilon}\left(\boldsymbol{w}_{h}\right) d \mathrm{~B}=\mathbf{W}^{\mathrm{T}}(\alpha \mathbf{M}+\beta \mathbf{K}) \dot{\mathbf{U}}=\mathbf{W}^{T} \mathbf{C} \dot{\mathbf{U}},
$$

with $\alpha$ and $\beta$ non-negative scalar values, and $\mathbf{W}$ nodal values of the test field. Selecting a Rayleigh damping for the present developments could be seen as a restriction, however this choice is retained in the vast majority of dynamical studies with the Finite Element Method (FEM) for engineering applications, mainly because it comes with convenient properties (e.g. positive definite damping matrix diagonalised by the same eigenvectors obtained from the generalised eigenvalue problem that stems from the undamped case). As a matter of fact, damping sources are very different in mechanical vibrations, see e.g. [45, 46, 47, 48, for general references and [49,50,51] for damping models in MEMS structures and their implementation in the FEM context often comes with case-by-case derivations and implementations.

Let us now consider the representation of the external forcing $\hat{\mathbf{F}}$ which acts on the mechanical system as either a surface traction or a body force. In this study, the external force will be expressed as a summation of harmonic components without constant terms:

$$
\hat{\mathbf{F}}=\sum_{j=1}^{\hat{\mathrm{n}}} \hat{\mathbf{F}}_{j}^{+} e^{+\mathrm{i} \Omega_{j} t}+\sum_{j=1}^{\hat{\mathrm{n}}} \hat{\mathbf{F}}_{j}^{-} e^{-\mathrm{i} \Omega_{j} t},
$$

where $\hat{\mathrm{n}}$ stands for the number of excitation frequencies, defined over a set $\boldsymbol{\Omega}$ and $\hat{\mathbf{F}}_{j}^{+}, \hat{\mathbf{F}}_{j}^{-}$are complexvalued vectors that will satisfy suitable constraints to make the forcing $\hat{\mathbf{F}}$ real-valued. It is worth stressing that the presence of complex conjugate pairs of imaginary exponents $\pm \mathfrak{i} \Omega_{j}$ is a prerequisite to respect this condition. The double summation is exploited in Section 5, where the real-valued structure of the forcing is leveraged to yield real-valued mappings and reduced dynamics coefficients. Alternatively, the following more concise expression can be used:

$$
\hat{\mathbf{F}}(t)=\sum_{j=1}^{2 \hat{\mathbf{n}}} \hat{\mathbf{F}}_{j} e^{\hat{\lambda}_{j} t},
$$

where $\hat{\mathbf{F}}_{j}$ spans over both $\hat{\mathbf{F}}_{j}^{+}$and $\hat{\mathbf{F}}_{j}^{-}$and $\hat{\lambda}_{j}$ represents both $+i \Omega_{j}$ and $-i \Omega_{j}$. The structure of $\hat{\lambda}_{j}$ is further detailed in Section 2.2 , where the linear properties of the problem are recalled, including the added frequencies due to the forcing.

Finally, the discretized finite element system considered in this work can be expressed as [52, 53$]$ :

$$
\mathbf{M} \ddot{\mathbf{U}}+\mathbf{C} \dot{\mathbf{U}}+\mathbf{K U}+\boldsymbol{G}(\mathbf{U}, \mathbf{U})+\boldsymbol{H}(\mathbf{U}, \mathbf{U}, \mathbf{U})=\varepsilon \sum_{j=1}^{2 \hat{\mathrm{n}}} \hat{\mathbf{F}}_{j} e^{\hat{\lambda}_{j} t},
$$

which is a system of $\mathrm{N}$ coupled nonlinear differential equations. Alternatively, one can adopt a first-order representation by introducing the velocity $\mathbf{V}$ as the time derivative of the displacement $\mathbf{U}$ :

$$
\begin{aligned}
& \mathbf{M} \dot{\mathbf{V}}+\mathbf{C V}+\mathbf{K U}+\boldsymbol{G}(\mathbf{U}, \mathbf{U})+\boldsymbol{H}(\mathbf{U}, \mathbf{U}, \mathbf{U})=\varepsilon \sum_{j=1}^{2 \hat{\mathbf{n}}} \hat{\mathbf{F}}_{j} e^{\hat{\lambda}_{j} t}, \\
& \mathbf{M} \dot{\mathbf{U}}-\mathbf{M V}=\mathbf{0} .
\end{aligned}
$$

Note that the choice to premultiply both sides of Eq. $111 \mathrm{~b}$ by the mass matrix is to ensure symmetry of the upcoming formulation as extensively detailed in [35]. In structural mechanics, $\mathrm{N}$ is typically large, hence computing periodic orbits for this system is prohibitively costly. Furthermore, stability analysis and continuation approaches are often computationally unfeasible, hence dimensionality reduction techniques play a crucial role. For $\hat{\mathbf{F}}=\mathbf{0}$, the system becomes autonomous and a high-order direct parametrisation of invariant manifolds for dimensionality reduction has already been proposed by the authors in [35. In the present work, we extend the treatment to the non-autonomous case and develop a high-order approach to the non-autonomous problem by extending the procedure initially introduced by Haro and de la Llave [39] and later applied for the first time in the context of mechanical systems by Breunung et al to problems described in the modal basis [30. 


\subsection{Spectral properties}

In this Section we focus on the main spectral properties associated with the problem at hand. In particular, the intricate link between the first-order problem and the second-order formulation will be specifically used in the remainder of the developments in order to simplify calculations.

Given the assumptions introduced in Section 2.1. Eq. 10 has at least a fixed point at the origin. For $\alpha, \beta=0$, the point is marginally stable, while for $\alpha, \beta>0$, the point is asymptotically stable. The linearized version of the autonomous part in Eq. 10 has the form:

$$
\mathbf{M U ̈}+\mathbf{C U}+\mathbf{K U}=\mathbf{0} .
$$

However, since $\mathbf{C}$ is defined as a linear combination of $\mathbf{M}$ and $\mathbf{K}$, real eigenfunctions and eigenfrequencies of system 12 can be obtained from the simpler generalised eigenvalue problem of the undamped system, i.e.:

$$
\left(-\omega_{j}^{2} \mathbf{M}+\mathbf{K}\right) \boldsymbol{\Phi}_{j}=\mathbf{0}
$$

For mechanical systems, $\mathbf{M}$ and $\mathbf{K}$ are symmetric positive definite in absence of rigid body motions. As a consequence, right and left eigenmodes coincide, and both $\boldsymbol{\Phi}_{j}$ and $\omega_{j}$ are real-valued. Eigenfunctions are mass-normalised and hence the following orthogonality properties hold:

$$
\boldsymbol{\Phi}_{i}^{T} \mathbf{M} \boldsymbol{\Phi}_{j}=\delta_{i j}, \quad \boldsymbol{\Phi}_{i}^{T} \mathbf{K} \boldsymbol{\Phi}_{j}=\omega_{i}^{2} \delta_{i j}
$$

Importantly, eigenfunctions $\boldsymbol{\phi}_{j}$ also solve the generalised eigenvalue problem for the damped system:

$$
\left(\Lambda_{j}^{2} \mathbf{M}+\Lambda_{j} \mathbf{C}+\mathbf{K}\right) \boldsymbol{\Phi}_{j}=\mathbf{0}
$$

while eigenvalues $\Lambda_{j}$ can be computed by projecting Eq. 15 on the modal basis $\boldsymbol{\Phi}_{i}$. Indeed, since Rayleigh damping is considered, we have that:

$$
\boldsymbol{\Phi}_{i}^{T} \mathbf{C \Phi}_{j}=\left(\alpha+\beta \omega_{j}^{2}\right) \delta_{i j}=2 \xi_{j} \omega_{j} \delta_{i j},
$$

with $\xi_{j}$ the modal damping coefficient, hereafter subjected to the condition of light damping, i.e. $\xi_{j}<1$. Eigenvalues are organised in complex conjugate pairs with expression $\Lambda_{j}=-\xi_{j} \omega_{j} \pm \mathfrak{i} \omega_{j} \sqrt{1-\xi_{j}^{2}}$, where $\mathfrak{i}$ is the imaginary unit. The complex eigenvalues $\Lambda_{j}$ are hence totally defined by the eigenfrequencies $\omega_{j}$ and the the Rayleigh damping coefficients $\alpha$ and $\beta$.

The procedure detailed in the present work is based on the PIM, which is formulated for dynamical systems written with first-order formulation in time. As a consequence, it is necessary to derive spectral properties of the first-order linearised system:

$$
\begin{aligned}
& \mathbf{M} \dot{\mathbf{V}}+\mathbf{C V}+\mathbf{K U}=\mathbf{0} \\
& \mathbf{M U}-\mathbf{M V}=\mathbf{0} .
\end{aligned}
$$

The first-order system, of dimension $2 \mathrm{~N}$, is non-symmetric and one needs to solve both right and left eigenvalue problems:

$$
\left(\Lambda_{i}\left[\begin{array}{cc}
\mathbf{M} & \mathbf{0} \\
\mathbf{0} & \mathbf{M}
\end{array}\right]+\left[\begin{array}{cc}
\mathbf{C} & \mathbf{K} \\
-\mathbf{M} & \mathbf{0}
\end{array}\right]\right) \mathbb{Y}_{i}=\mathbf{0}, \quad \mathbb{X}_{i}^{\mathrm{T}}\left(\Lambda_{i}\left[\begin{array}{cc}
\mathbf{M} & \mathbf{0} \\
\mathbf{0} & \mathbf{M}
\end{array}\right]+\left[\begin{array}{cc}
\mathbf{C} & \mathbf{K} \\
-\mathbf{M} & \mathbf{0}
\end{array}\right]\right)=\mathbf{0}
$$

with $\mathbb{Y}$ and $\mathbb{X}$ right and left eigenvectors, respectively. Eigenvalues are the same as those obtained from Eq. 15, and they can be ordered as:

$$
\begin{aligned}
& \forall j=1, \ldots, \mathrm{N} \\
& \Lambda_{j} \triangleq-\xi_{j} \omega_{j}+\mathfrak{i} \omega_{j} \sqrt{1-\xi_{j}^{2}} \\
& \Lambda_{j+\mathrm{N}}=\bar{\Lambda}_{j} \triangleq-\xi_{j} \omega_{j}-\mathfrak{i} \omega_{j} \sqrt{1-\xi_{j}^{2}}
\end{aligned}
$$

where the bar denotes the complex conjugate operator. For mechanical vibratory problems, eigenvalues come in pairs of complex conjugates, which reflects the fact that the original problem is second-order in time, and that the velocity is needed as independent variable. Consequently manifolds associated to linear modes or NNMs are essentially even-dimensional, in order to allow the birth of periodic orbits representing the oscillations [10,54,5]. The eigenfunctions that result from Eq. [18] are: 


$$
\begin{aligned}
& \forall j=1, \ldots, \mathrm{N} \\
& \mathbb{X}_{j} \triangleq \frac{1}{\Lambda_{j}-\bar{\Lambda}_{j}}\left[\begin{array}{c}
\boldsymbol{\Phi}_{j} \\
-\boldsymbol{\Phi}_{j} \bar{\Lambda}_{j},
\end{array}\right], \quad \mathbb{Y}_{j} \triangleq\left[\begin{array}{c}
\boldsymbol{\Phi}_{j} \Lambda_{j} \\
\boldsymbol{\Phi}_{j}
\end{array}\right], \\
& \mathbb{X}_{j+\mathrm{N}}=\overline{\mathbb{X}}_{j} \triangleq \frac{1}{\bar{\Lambda}_{j}-\Lambda_{j}}\left[\begin{array}{c}
\boldsymbol{\Phi}_{j} \\
-\boldsymbol{\Phi}_{j} \Lambda_{j}
\end{array}\right], \quad \mathbb{Y}_{j+\mathrm{N}}=\overline{\mathbb{Y}}_{j} \triangleq\left[\begin{array}{c}
\boldsymbol{\Phi}_{j} \bar{\Lambda}_{j} \\
\boldsymbol{\Phi}_{j}
\end{array}\right] .
\end{aligned}
$$

These expressions highlight once again that the solution to the standard eigenvalue problem for the secondorder conservative problem provides all the quantities required to define eigenvectors and eigenvalues of the first order formulation. These eigenfunctions satisfy the following orthogonality properties:

$$
\mathbb{X}_{i}^{\mathrm{T}}\left[\begin{array}{cc}
\mathbf{M} & \mathbf{0} \\
\mathbf{0} & \mathbf{M}
\end{array}\right] \mathbb{Y}_{j}=\delta_{i j}, \quad \mathbb{X}_{i}^{\mathrm{T}}\left[\begin{array}{cc}
\mathbf{C} & \mathbf{K} \\
-\mathbf{M} & \mathbf{0}
\end{array}\right] \mathbb{Y}_{j}=-\Lambda_{j} \delta_{i j}
$$

It is worth stressing that the parametrization procedure, detailed in the following Sections, does not require the computation of the whole modal basis, but only of the $\mathrm{n} \ll N$ selected master modes $\left\{\boldsymbol{\Phi}_{m_{1}}, \boldsymbol{\Phi}_{m_{2}}, \ldots, \boldsymbol{\Phi}_{m_{\mathrm{n}}}\right\}$ with complex eigenvalues $\left\{\Lambda_{m_{1}}, \Lambda_{m_{2}}, \ldots, \Lambda_{m_{\mathrm{n}}}\right\}$.

The spectral properties associated to the master modes are collected in the matrices:

$$
\begin{aligned}
& \boldsymbol{\phi} \triangleq\left[\boldsymbol{\Phi}_{m_{1}}, \boldsymbol{\Phi}_{m_{2}}, \ldots, \boldsymbol{\Phi}_{m_{\mathrm{n}}}, \boldsymbol{\Phi}_{m_{1}}, \boldsymbol{\Phi}_{m_{2}}, \ldots, \boldsymbol{\Phi}_{m_{\mathrm{n}}}\right], \\
& \mathbf{X} \triangleq\left[\mathbb{X}_{m_{1}}, \mathbb{X}_{m_{2}}, \ldots, \mathbb{X}_{m_{\mathrm{n}}}, \overline{\mathbb{X}}_{m_{1}}, \overline{\mathbb{X}}_{m_{2}}, \ldots, \overline{\mathbb{X}}_{m_{\mathrm{n}}}\right], \\
& \mathbf{Y} \triangleq\left[\mathbb{Y}_{m_{1}}, \mathbb{Y}_{m_{2}}, \ldots, \mathbb{Y}_{m_{\mathrm{n}}}, \overline{\mathbb{Y}}_{m_{1}}, \overline{\mathbb{Y}}_{m_{2}}, \ldots, \overline{\mathbb{Y}}_{m_{\mathrm{n}}}\right], \\
& \boldsymbol{\lambda} \triangleq \operatorname{diag}\left[\Lambda_{m_{1}}, \Lambda_{m_{2}}, \ldots, \Lambda_{m_{\mathrm{n}}}, \bar{\Lambda}_{m_{1}}, \bar{\Lambda}_{m_{2}}, \ldots, \bar{\Lambda}_{m_{\mathrm{n}}}\right],
\end{aligned}
$$

all having $2 \mathrm{n}$ columns, for reasons that will be clarified in the course of the development (see e.g. Eq. (35)). Moreover, in what follows, the symbols $\boldsymbol{\phi}_{j}, \mathbf{X}_{j}, \mathbf{Y}_{j}$ will be used to denote at the $j$-th column in the respective matrices, and $\lambda_{j}$ for the $j$-th diagonal coefficient in $\boldsymbol{\lambda}$.

Similarly, and as anticipated in Section 2.1, it is possible to define a specific ordering for the of complex eigenvalues $\hat{\lambda}_{j}$ related to the frequencies introduced by the external forcing. Such eigenvalues can be organised with the same ordering as the one used for the autonomous problem:

$$
\begin{aligned}
& \forall j=1, \ldots, \hat{\mathrm{n}}, \\
& \hat{\lambda}_{j} \triangleq+\mathrm{i} \Omega_{j}, \\
& \hat{\lambda}_{j+\hat{\mathrm{n}}}=\overline{\hat{\lambda}}_{j} \triangleq-\mathrm{i} \Omega_{j} .
\end{aligned}
$$

This is associated with the fact that the forced (non-autonomous) system can be recast as an autonomous one by introducing a set of auxiliary linear oscillator equations, the solutions to which being the sine and cosine functions.

$$
\begin{aligned}
& \forall j=1, \ldots, \hat{\mathrm{n}}, \\
& \dot{\hat{\mathrm{a}}}_{j}=\Omega_{j} \hat{\mathrm{a}}_{j+\hat{\mathrm{n}}}, \\
& \dot{\hat{\mathrm{a}}}_{j+\hat{\mathrm{n}}}=-\Omega_{j} \hat{\mathrm{a}}_{j},
\end{aligned}
$$

with $\hat{a}_{j}$ real-valued auxiliary variables. Generally, nonlinear terms are added to Eqs. (24) to ensure that their unique limit cycle is the unitary circle (see e.g. [55] and references therein), but for the purpose of the present explanation such constraint is not required. The linear part of this last set of equations is uncoupled for each harmonic term and its block antidiagonal structure yields the conjugate pair of eigenvalues described by Eq. 23.

Finally, for future use, we also introduce the projection of the displacement onto the modes. The modal displacement $\mathrm{u}_{j}$ and velocity $\mathrm{v}_{j}$ are defined as:

$$
\mathrm{u}_{j}=\boldsymbol{\Phi}_{j} \mathbf{M U}, \quad \mathrm{v}_{j}=\boldsymbol{\Phi}_{j} \mathbf{M V}
$$




\section{Parametrisation method for time-dependent manifolds}

The aim of the present Section is to adapt the direct parametrisation method in order to take into account the external forcing. The resulting time-dependent invariant manifolds will thus contain the periodic orbits of the forced system and will allow the derivation of efficient ROMs. The effect of the forcing might result in deformations of the whisker that will be computed with a dedicated expansion. The methodology has been first adapted to vibratory mechanical systems in [42, and used for model order reduction in [43, according to the general guidelines given in 40,41. Following the same line of research, the method is here adapted to allow for a direct computation eliminating the need to compute the full modal basis as a first step. We start by detailing the general methodology in the next Section. Then the solution to the autonomous part, which follows the calculations presented in [35], is recalled, and finally the non-autonomous part is solved at generic order.

\subsection{General framework}

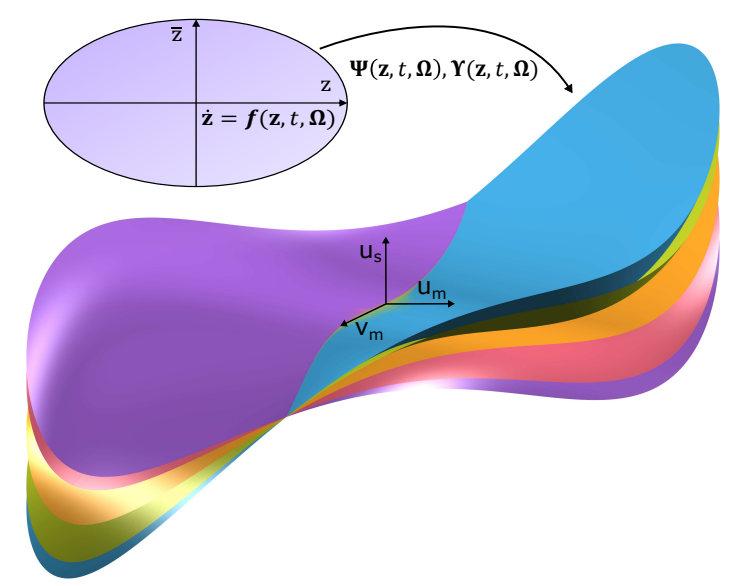

Fig. 1 Qualitative representation of the time-dependent invariant manifold (or whisker) for a fictitious problem. Upon parametrisation of a two-dimensional invariant set the reduced dynamics is flat (purple ellipsoid) and pairs of normal coordinates values are mapped through time dependent laws $\boldsymbol{\Psi}, \boldsymbol{\Upsilon}$ to the physical space. The reported hypersurfaces represent the physical configuration of the whisker at different time instants. For mechanical systems subjected to harmonic forcing, the two-dimensional whisker undergoes harmonic motion in physical space with instantaneous configuration defined by the phase $\Theta$. On the other hand, the reduced dynamics results non-autonomous but it retains its two-dimensional planar structure as for the autonomous case. $(\square),(\square),(\square),(\square),(\square)$ colors correspond to a phase $\Theta=\Omega t$ equal to $0, \pi / 4, \pi / 2,3 \pi / 4$, and $\pi$, respectively.

The general idea of the parametrisation method for invariant manifolds is to introduce a nonlinear change of coordinates between the physical space variables $\mathbf{U}, \mathbf{V}$ and the new normal coordinates $\mathbf{z}$. These normal coordinates, with dimension $2 \mathrm{n}$, describe the motion on an invariant manifold, which can be seen as the nonlinear continuation of the eigenspace spanned by the $\mathrm{n}$ master modes. Being the dimension of the invariant set much smaller than that of the original problem, we have $\mathrm{n} \ll \mathrm{N}$. Moreover, the dimension of the reduction subspace is always an even number (thus $2 \mathrm{n}$ ) of paired reduced coordinates, to reflect the fact that oscillations come with complex conjugate eigenvalues as also done for example in [18, 29, 35].

Using our initial scaling of the external forcing with the book-keeping parameter $\varepsilon$, the main idea is to split the searched nonlinear mappings into two terms as:

$$
\begin{aligned}
& \mathbf{U}=\boldsymbol{\Psi}(\mathbf{z}, \boldsymbol{\Omega}, t)=\boldsymbol{\Psi}(\mathbf{z})+\varepsilon \hat{\boldsymbol{\Psi}}(\mathbf{z}, \boldsymbol{\Omega}, t)+O\left(\varepsilon^{2}\right), \\
& \mathbf{V}=\boldsymbol{\Upsilon}(\mathbf{z}, \boldsymbol{\Omega}, t)=\boldsymbol{\Upsilon}(\mathbf{z})+\varepsilon \hat{\boldsymbol{\Upsilon}}(\mathbf{z}, \boldsymbol{\Omega}, t)+O\left(\varepsilon^{2}\right)
\end{aligned}
$$

In these equations, two different nonlinear mappings, respectively for the displacement $\mathbf{U}$ and the velocity $\mathbf{V}$, are introduced, following [33, 35, since it has been shown that such splitting allows a better understanding of the relationships between variables as well as a reduction of the computational burden. Secondly, the $\varepsilon^{0}$ terms $\boldsymbol{\Psi}(\mathbf{z})$ and $\boldsymbol{\Upsilon}(\mathbf{z})$ are related to the autonomous problems, and their computation has already been tackled in 35]. In addition, the new terms $\hat{\boldsymbol{\Psi}}(\mathbf{z}, \boldsymbol{\Omega}, t)$ and $\hat{\boldsymbol{\Upsilon}}(\mathbf{z}, \boldsymbol{\Omega}, t)$ represent the first-order contribution of the forcing on the time-dependent manifold, and the main aim of the present article is to detail their 
direct computation, at generic order. The reduced dynamics along the whisker, which is also unknown at this stage, is then expressed as:

$$
\dot{\mathbf{z}}=\boldsymbol{f}(\mathbf{z})+\varepsilon \hat{\boldsymbol{f}}(\mathbf{z}, \boldsymbol{\Omega}, t)+O\left(\varepsilon^{2}\right),
$$

where again the splitting between autonomous and non-autonomous parts has been expressed. In this last expression, $\boldsymbol{f}(\mathbf{z})$ is the vector field obtained from the autonomous problem, and $\hat{\boldsymbol{f}}(\mathbf{z}, \boldsymbol{\Omega}, t)$ is the new contribution to the dynamics that will be introduced by taking into account the external forcing in the parametrisation procedure.

Two remarks are worth mentioning here. First, the role of the $\varepsilon$ book-keeping parameter is mainly to separate, in the calculations, the terms according to their nature (autonomous vs non-autonomous). As it is customary done in such perturbative development [56], the parameter is set to 1 at the end of the process and the smallness of the forcing term is incorporated in its value. Consequently, once the theoretical calculations done and the numerical results analyzed, in Section $6, \varepsilon=1$ will be set, and the smallness of the forcing will be quantified by introducing a meaningful scaling on the amplitude of the external force.

Secondly, one can remark that the mappings are here introduced for the nodal coordinates of the FE formulation $(\mathbf{U}, \mathbf{V})$, i.e. by taking as a starting point the semi-discrete problem, which is the most customary approach to deal with general FE problems for mechanical systems. However, similar results can be obtained by introducing a coordinate change for the continuous displacement and velocity fields $\boldsymbol{u}$ and $\boldsymbol{v}$. By following this approach, the resulting invariance equations are elliptic differential equations that can be solved with numerical approaches such as the finite element method. Details of this alternative derivation are reported in Appendix A

In order to solve for the unknowns, which are respectively the nonlinear mappings and the reduced dynamics, one derives the invariance equation of the searched manifold by eliminating the dependence with respect to time. The first step is to differentiate Eqs. 26) with respect to time and use Eq. (27) to yield:

$$
\begin{aligned}
& \dot{\mathbf{U}}=\nabla_{\mathbf{z}} \boldsymbol{\Psi}(\mathbf{z}) \boldsymbol{f}(\mathbf{z})+\varepsilon\left(\dot{\hat{\boldsymbol{\Psi}}}(\mathbf{z}, \boldsymbol{\Omega}, t)+\nabla_{\mathbf{z}} \hat{\boldsymbol{\Psi}}(\mathbf{z}, \boldsymbol{\Omega}, t) \boldsymbol{f}(\mathbf{z})+\nabla_{\mathbf{z}} \boldsymbol{\Psi}(\mathbf{z}) \hat{\boldsymbol{f}}(\mathbf{z}, \boldsymbol{\Omega}, t)\right)+O\left(\varepsilon^{2}\right), \\
& \dot{\mathbf{V}}=\nabla_{\mathbf{z}} \boldsymbol{\Upsilon}(\mathbf{z}) \boldsymbol{f}(\mathbf{z})+\varepsilon\left(\dot{\hat{\boldsymbol{\varphi}}}(\mathbf{z}, \boldsymbol{\Omega}, t)+\nabla_{\mathbf{z}} \hat{\boldsymbol{\Upsilon}}(\mathbf{z}, \boldsymbol{\Omega}, t) \boldsymbol{f}(\mathbf{z})+\nabla_{\mathbf{z}} \boldsymbol{\Upsilon}(\mathbf{z}) \hat{\boldsymbol{f}}(\mathbf{z}, \boldsymbol{\Omega}, t)\right)+O\left(\varepsilon^{2}\right),
\end{aligned}
$$

with $\nabla_{\mathbf{z}}(\cdot)$ denoting the gradient with respect to the normal coordinates. Upon substitution of mappings and their derivatives in Eq. (11), one can derive for each order of $\varepsilon$ a set of invariance equations whose unknowns are estimated order by order in a hierarchical structure. For $\varepsilon^{0}$, the invariance equations associated to the autonomous system read:

$$
\begin{aligned}
& \mathbf{M} \nabla_{\mathbf{z}} \boldsymbol{\Upsilon}(\mathbf{z}) \boldsymbol{f}(\mathbf{z})+\mathbf{C} \boldsymbol{\Psi}(\mathbf{z})+\mathbf{K} \boldsymbol{\Psi}(\mathbf{z})+\boldsymbol{G}(\boldsymbol{\Psi}(\mathbf{z}), \boldsymbol{\Psi}(\mathbf{z}))+\boldsymbol{H}(\boldsymbol{\Psi}(\mathbf{z}), \boldsymbol{\Psi}(\mathbf{z}), \boldsymbol{\Psi}(\mathbf{z}))=\mathbf{0}, \\
& \mathbf{M} \nabla_{\mathbf{z}} \boldsymbol{\Psi}(\mathbf{z}) \boldsymbol{f}(\mathbf{z})-\mathbf{M} \boldsymbol{\Upsilon}(\mathbf{z})=\mathbf{0} .
\end{aligned}
$$

These equations have already been obtained and solved at arbitrary order of expansion in [35. At order $\varepsilon^{1}$, time-dependent terms associated to the parametrisation of the non-autonomous system appear:

$$
\begin{aligned}
& \mathbf{M} \dot{\hat{\boldsymbol{\Upsilon}}}(\mathbf{z})+\mathbf{M} \nabla_{\mathbf{z}} \hat{\boldsymbol{\Upsilon}}(\mathbf{z}, \boldsymbol{\Omega}, t) \boldsymbol{f}(\mathbf{z})+\mathbf{M} \nabla_{\mathbf{z}} \boldsymbol{\Upsilon}(\mathbf{z}) \hat{\boldsymbol{f}}(\mathbf{z}, \boldsymbol{\Omega}, t)+\mathbf{C} \hat{\boldsymbol{\Upsilon}}(\mathbf{z}, \boldsymbol{\Omega}, t) \\
& +\mathbf{K} \hat{\boldsymbol{\Psi}}(\mathbf{z}, \boldsymbol{\Omega}, t)+2 \boldsymbol{G}(\hat{\boldsymbol{\Psi}}(\mathbf{z}, \boldsymbol{\Omega}, t), \boldsymbol{\Psi}(\mathbf{z}))+3 \boldsymbol{H}(\hat{\boldsymbol{\Psi}}(\mathbf{z}, \boldsymbol{\Omega}, t), \boldsymbol{\Psi}(\mathbf{z}), \boldsymbol{\Psi}(\mathbf{z}))=\sum_{j=1}^{2 \hat{\mathrm{n}}} \hat{\mathbf{F}}_{j} e^{\hat{\lambda}_{j} t}, \\
& \mathbf{M} \dot{\hat{\boldsymbol{\Psi}}}(\mathbf{z})+\mathbf{M} \nabla_{\mathbf{z}} \hat{\boldsymbol{\Psi}}(\mathbf{z}, \boldsymbol{\Omega}, t) \boldsymbol{f}(\mathbf{z})+\mathbf{M} \nabla_{\mathbf{z}} \boldsymbol{\Psi}(\mathbf{z}) \hat{\boldsymbol{f}}(\mathbf{z}, \boldsymbol{\Omega}, t)-\mathbf{M} \hat{\boldsymbol{\Upsilon}}(\mathbf{z}, \boldsymbol{\Omega}, t)=\mathbf{0},
\end{aligned}
$$

where the symmetries of the $\boldsymbol{G}$ and $\boldsymbol{H}$ operators have been exploited to obtain a more compact expression. Under the assumption that the $\varepsilon^{0}$-invariance equations have been solved and that $\boldsymbol{\Psi}, \boldsymbol{\Upsilon}$, and $\boldsymbol{f}$ are known, Eq. (30) is a set of time-dependent linear ordinary differential equations in the unknown variables $\hat{\boldsymbol{\Psi}}, \hat{\boldsymbol{\Upsilon}}$, and $\boldsymbol{f}$. This represents an important property of Eq. (30), which can be used to search for solutions with finite Fourier expansions to represent all unknown variables of the system. Furthermore, thanks again to linearity, the solution will have the same harmonic content as that of the external forcing and hence the following expansions hold:

$$
\begin{aligned}
& \hat{\boldsymbol{\Psi}}(\mathbf{z}, \boldsymbol{\Omega}, t)=\sum_{j=1}^{2 \hat{\mathrm{n}}} \hat{\boldsymbol{\Psi}}_{j}(\mathbf{z}) e^{\hat{\lambda}_{j} t} \\
& \hat{\boldsymbol{\Upsilon}}(\mathbf{z}, \boldsymbol{\Omega}, t)=\sum_{j=1}^{2 \hat{\mathrm{n}}} \hat{\boldsymbol{\Upsilon}}_{j}(\mathbf{z}) e^{\hat{\lambda}_{j} t} \\
& \hat{\boldsymbol{f}}(\mathbf{z}, \boldsymbol{\Omega}, t)=\sum_{j=1}^{2 \hat{\mathbf{n}}} \hat{\boldsymbol{f}}_{j}(\mathbf{z}) e^{\hat{\lambda}_{j} t}
\end{aligned}
$$


making appear the unknowns $\hat{\boldsymbol{\Psi}}_{j}(\mathbf{z}), \hat{\boldsymbol{\Upsilon}}_{j}(\mathbf{z})$, and $\hat{\boldsymbol{f}}_{j}(\mathbf{z})$ as Fourier coefficients. Each term depends on the normal coordinates only, hence dropping the explicit dependence on time. Upon substitution of Eq. (31) in Eq. (30), an algebraic version of the invariance equation at order $\varepsilon^{1}$ is retrieved. The resulting $\varepsilon^{1}$-invariance equations are then finally expressed as:

$$
\begin{aligned}
& \forall j=1, \ldots, 2 \hat{\mathrm{n}}, \\
& \hat{\lambda}_{j} \mathbf{M} \hat{\boldsymbol{\Upsilon}}_{j}(\mathbf{z})+\mathbf{M} \nabla_{\mathbf{z}} \hat{\boldsymbol{\Psi}}_{j}(\mathbf{z}) \boldsymbol{f}(\mathbf{z})+\mathbf{M} \nabla_{\mathbf{z}} \boldsymbol{\Upsilon}(\mathbf{z}) \hat{\boldsymbol{f}}_{j}(\mathbf{z})+\mathbf{C} \hat{\boldsymbol{\Upsilon}}_{j}(\mathbf{z}) \\
& +\mathbf{K} \hat{\boldsymbol{\Psi}}_{j}(\mathbf{z})+2 \boldsymbol{G}\left(\hat{\boldsymbol{\Psi}}_{j}(\mathbf{z}), \boldsymbol{\Psi}(\mathbf{z})\right)+3 \boldsymbol{H}\left(\hat{\boldsymbol{\Psi}}_{j}(\mathbf{z}), \boldsymbol{\Psi}(\mathbf{z}), \boldsymbol{\Psi}(\mathbf{z})\right)=\hat{\mathbf{F}}_{j}, \\
& \hat{\lambda}_{j} \mathbf{M} \hat{\boldsymbol{\Psi}}_{j}(\mathbf{z})+\mathbf{M} \nabla_{\mathbf{z}} \hat{\boldsymbol{\Psi}}_{j}(\mathbf{z}) \boldsymbol{f}(\mathbf{z})+\mathbf{M} \nabla_{\mathbf{z}} \boldsymbol{\Psi}(\mathbf{z}) \hat{\boldsymbol{f}}_{j}(\mathbf{z})-\mathbf{M} \hat{\boldsymbol{\Upsilon}}_{j}(\mathbf{z})=\mathbf{0} .
\end{aligned}
$$

These equations can be solved using the general procedure detailed in [35, since the structure of the problem at hand is very similar. In the following Section, this procedure, based on an asymptotic polynomial expansion at arbitrary order, is first briefly recalled. As a distinctive feature of the time-dependent problem, both nonlinear mappings and reduced dynamics show an explicit dependence on forcing vectors $\hat{\mathbf{F}}_{j}$ and excitation frequencies $\Omega_{i}$. Consequently, the solution should in principle be computed for every value of those two parameters, as already remarked in [38,43. This requirement drastically limits the applicability of the method for model-order reduction, since one of the main goal is generally to derive a ROM able to reproduce the nonlinear frequency response curves (FRCs) with varying values of excitation frequencies and amplitudes, as reported in [22,30,43,36], where the deformation of the whisker has not been taken into account. In order to cope with this limitation, it is here assumed that both unknowns (nonlinear mappings and reduced dynamics) show a mild dependency on these parameters. As a consequence, a single parametrisation procedure is realized, and assumed to be valid for a given frequency window $\left[\Omega_{i}-\right.$ $\left.\delta \Omega, \Omega_{i}+\delta \Omega\right]$. The resulting ROM can thus be used for parametric studies and directly solved with numerical continuation techniques to compute the FRCs of the structure. The validity of such assumptions will be checked and discussed with dedicated numerical examples in Section 6

\subsection{Invariance equations}

This Section is devoted to detail the solutions to the invariance equations at generic order. The process relies on introducing an asymptotic expansion into both autonomous and non-autonomous invariance equations (29) and (32). The arbitrary order solution to the $\varepsilon^{0}$-invariance equation has already been detailed for example in [35], following the general guidelines provided in [28], but proposing a direct solution from the physical space, as also shown in [34. This important derivation is first briefly recalled in Section 3.2.1. Then the direct solution, from the physical coordinate, to the $\varepsilon^{1}$-invariance equation is detailed in Section 3.2 .2 This constitutes the main novelty of the present developments. Whereas general solutions for vibratory systems have already been detailed in [42,43, the computations were operated from the modal space, and thus not directly applicable to FE structures. A direct approach is here proposed. Emphasis is set to the novel class of resonance conditions associated to the presence of forcing terms.

\subsection{1 $\varepsilon^{0}$-invariance equations}

The arbitrary order solution to the autonomous problem is first recalled, following [35. Taylor expansions of both mappings $\boldsymbol{\Psi}(\mathbf{z}), \boldsymbol{\Upsilon}(\mathbf{z})$, and reduced dynamics $\boldsymbol{f}(\mathbf{z})$ as function of the normal coordinate $\mathbf{z}$, are introduced as:

$$
\boldsymbol{\Psi}(\mathbf{z})=\sum_{p=0}^{o}[\boldsymbol{\Psi}(\mathbf{z})]_{p}, \quad \boldsymbol{\Upsilon}(\mathbf{z})=\sum_{p=0}^{o}[\boldsymbol{\Upsilon}(\mathbf{z})]_{p}, \quad \boldsymbol{f}(\mathbf{z})=\sum_{p=0}^{o}[\boldsymbol{f}(\mathbf{z})]_{p} .
$$

The adopted notation follows that used in 35 , and is briefly summarized in Appendix B. In particular, o stands for the maximum order of the computed expansion, and []$_{p}$ represents a collection of polynomial terms of order $p$. Each order of the polynomial expansions can be explicitly formulated as:

$$
[\boldsymbol{\Psi}(\mathbf{z})]_{p}=\sum_{\mathcal{I}} \boldsymbol{\Psi}_{\mathcal{I}}^{(p)} \pi_{\mathcal{I}}^{(p)}, \quad[\boldsymbol{\Upsilon}(\mathbf{z})]_{p}=\sum_{\mathcal{I}} \boldsymbol{\Upsilon}_{\mathcal{I}}^{(p)} \pi_{\mathcal{I}}^{(p)}, \quad[\boldsymbol{f}(\mathbf{z})]_{p}=\sum_{\mathcal{I}} \mathbf{f}_{\mathcal{I}}^{(p)} \pi_{\mathcal{I}}^{(p)}
$$

In these expressions, a compact notation for the the monomials has been used, where $\mathcal{I}$ is an arbitrary set of $p$ indexes, $\mathcal{I}=\left\{i_{1} i_{2} \ldots i_{p}\right\}$, and $\pi_{\mathcal{I}}^{(p)}$ stands for the monomial associated to $\mathcal{I}: \pi_{\mathcal{I}}^{(p)}=z_{i_{1}} z_{i_{2}} \ldots z_{i_{p}}$. The superscript $(\cdot)^{(p)}$ denotes the order of both the unknown vectors of coefficients and the monomial.

Since the forcing is assumed to have no constant part, the origin of the phase space (representing the structure at rest) is a fixed point. As a consequence, the order $p=0$ in the expansions $(33)$ is vanishing. 
The first-order terms are also easy to derive, see e.g. 35] for a detailed presentation, as the mappings have to be identity-tangent to the set of $\mathrm{n}$ master modes.

The solution to the first-order homological equations writes (see the notation introduced in Eq. (22)):

$$
[\boldsymbol{\Psi}(\mathbf{z})]_{1}=\boldsymbol{\phi}, \quad[\boldsymbol{r}(\mathbf{z})]_{1}=\boldsymbol{\lambda} \boldsymbol{\phi}, \quad[\boldsymbol{f}(\mathbf{z})]_{1}=\boldsymbol{\lambda} .
$$

It should be noted that Eq. (35) sets a correspondence between the normal coordinates and the master modes in the sense that, according to Eq. $\left[22, z_{i}\right.$ and $z_{i+\mathrm{n}}$ are both associated to the $i$-th master mode $\boldsymbol{\Phi}_{i}$, but correspond to complex conjugate eigenvectors and eigenvalues of the first-order system.

Consequently the unknowns of the expansions start from order 2, and the nonlinear mappings and reduced dynamics can be rewritten as

$$
\begin{aligned}
& \boldsymbol{\Psi}(\mathbf{z})=\boldsymbol{\phi} \mathbf{z}+\sum_{p=2}^{o}[\boldsymbol{\Psi}(\mathbf{z})]_{p}=\sum_{i=1}^{2 \mathrm{n}} \boldsymbol{\phi}_{i} \mathbf{z}_{i}+\sum_{p=2}^{o}[\boldsymbol{\Psi}(\mathbf{z})]_{p} \\
& \boldsymbol{\Upsilon}(\mathbf{z})=\boldsymbol{\lambda} \boldsymbol{\phi} \mathbf{z}+\sum_{p=2}^{o}[\boldsymbol{\Upsilon}(\mathbf{z})]_{p}=\sum_{i=1}^{2 \mathrm{n}} \lambda_{i} \boldsymbol{\phi}_{i} \mathrm{z}_{i}+\sum_{p=2}^{o}[\boldsymbol{\Upsilon}(\mathbf{z})]_{p} \\
& \boldsymbol{f}(\mathbf{z})=\boldsymbol{\lambda} \mathbf{z}+\sum_{p=2}^{o}[\boldsymbol{f}(\mathbf{z})]_{p} .
\end{aligned}
$$

The general expressions for the solution to the order- $p$ homological equation are now recalled, for an arbitrary order $p \geq 2$. Let us first denote as $\mathcal{H}^{(p)}$ the collection of all possible sets $\mathcal{I}$ for a given expansion order $p$. As detailed in [35], the order- $p$ homological equation can be written for any monomial term $\pi_{\mathcal{I}}$ as:

$$
\begin{aligned}
& \forall p \in\{2, \ldots, o\}, \quad \forall \mathcal{I} \in \mathcal{H}^{(p)}, \\
& \sigma_{\mathcal{I}} \mathbf{M} \mathbf{\Upsilon}_{\mathcal{I}}^{(p)}+\mathbf{C} \boldsymbol{\Upsilon}_{\mathcal{I}}^{(p)}+\mathbf{K} \Psi_{\mathcal{I}}^{(p)}+\sum_{s=1}^{2 \mathrm{n}}\left(\lambda_{s} f_{s \mathcal{I}}^{(p)} \mathbf{M} \boldsymbol{\phi}_{s}\right)+\mathbf{M} \boldsymbol{\nu}_{\mathcal{I}}^{(p)}+\mathbf{G}_{\mathcal{I}}^{(p)}+\mathbf{H}_{\mathcal{I}}^{(p)}=\mathbf{0} \\
& \sigma_{\mathcal{I}} \mathbf{M} \Psi_{\mathcal{I}}^{(p)}-\mathbf{M} \boldsymbol{\Upsilon}_{\mathcal{I}}^{(p)}+\sum_{s=1}^{2 \mathrm{n}}\left(f_{s \mathcal{I}}^{(p)} \mathbf{M} \boldsymbol{\phi}_{s}\right)+\mathbf{M} \boldsymbol{\mu}_{\mathcal{I}}^{(p)}=\mathbf{0}
\end{aligned}
$$

All quantities reported in Eq. (37) are defined as:

$$
\begin{aligned}
\sigma_{\mathcal{I}} & =\lambda_{i_{1}}+\lambda_{i_{2}}+\ldots+\lambda_{i_{p}}, \\
\boldsymbol{\mu}_{\mathcal{I}}^{(p)} & =\sum_{s=1}^{2 \mathrm{n}} \sum_{k=2}^{p-1} \sum_{l=0}^{p-k} \boldsymbol{\Psi}_{\left\{i_{1} \ldots i_{l} s i_{l+k+1} \ldots i_{p}\right\}}^{(p-k+1)} f_{s\left\{i_{l+1} \ldots i_{l+k}\right\}}^{(k)}, \\
\boldsymbol{\nu}_{\mathcal{I}}^{(p)} & =\sum_{s=1}^{2 \mathrm{n}} \sum_{k=2}^{p-1} \sum_{l=0}^{p-k} \boldsymbol{\Upsilon}_{\left\{i_{1} \ldots i_{l} s i_{l+k+1} \ldots i_{p}\right\}}^{(p-k+1)} f_{s\left\{i_{l+1} \ldots i_{l+k}\right\}}^{(k)}, \\
\mathbf{G}_{\mathcal{I}}^{(p)} & =\sum_{k=1}^{p-1} \boldsymbol{G}\left(\boldsymbol{\Psi}_{\left\{i_{1} \ldots i_{k}\right\}}^{(k)}, \boldsymbol{\Psi}_{\left\{i_{k+1} \ldots i_{p}\right\}}^{(p-k)}\right), \\
\mathbf{H}_{\mathcal{I}}^{(p)} & =\sum_{k=1}^{p-2} \sum_{l=1}^{p-1} \boldsymbol{H}\left(\boldsymbol{\Psi}_{\left\{i_{1} \ldots i_{k}\right\}}^{(k)}, \Psi_{\left\{i_{k+1} \ldots i_{k+1}\right\}}^{(l)}, \Psi_{\left\{i_{k+1+1} \ldots i_{p}\right\}}^{(p-k-l)}\right),
\end{aligned}
$$

where the terms $\mathbf{G}_{\mathcal{I}}^{(p)}$ and $\mathbf{H}_{\mathcal{I}}^{(p)}$ are computed as reported in Appendix $\mathrm{C}$ It is worth stressing that all the subscripts $i_{n}$ belong to the set $\mathcal{I}=\left\{i_{1}, i_{2}, \ldots, i_{p}\right\}$ that defines the monomial term $\pi_{\mathcal{I}}^{(p)}$ considered in Eq. (37). It is possible to use a more convenient matrix representation of Eq. (37):

$$
\begin{aligned}
& \forall p \in\{2, \ldots, o\}, \quad \forall \mathcal{I} \in \mathcal{H}^{(p)}, \\
& \left(\sigma_{\mathcal{I}}\left[\begin{array}{cc}
\mathbf{M} & \mathbf{0} \\
\mathbf{0} & \mathbf{M}
\end{array}\right]+\left[\begin{array}{cc}
\mathbf{C} & \mathbf{K} \\
-\mathbf{M} & \mathbf{0}
\end{array}\right]\right)\left[\begin{array}{l}
\mathbf{\Upsilon}_{\mathcal{I}}^{(p)} \\
\mathbf{\Psi}_{\mathcal{I}}^{(p)}
\end{array}\right]+\sum_{s=1}^{2 n} f_{s \mathcal{I}}^{(p)}\left[\begin{array}{cc}
\mathbf{M} & \mathbf{0} \\
\mathbf{0} & \mathbf{M}
\end{array}\right] \mathbf{Y}_{s}=\left[\begin{array}{c}
-\mathbf{M} \boldsymbol{\nu}_{\mathcal{I}}^{(p)}-\mathbf{G}_{\mathcal{I}}^{(p)}-\mathbf{H}_{\mathcal{I}}^{(p)} \\
-\mathbf{M} \boldsymbol{\mu}_{\mathcal{I}}^{(p)}
\end{array}\right] .
\end{aligned}
$$

Detailed expressions for the solutions to Eq. (39) are much easier to derive when the problem is projected onto the modal space, as recommended for example in [28, since when operating from the physical space, all the unknowns are interwoven. Projecting on the master modes yields the so-called tangent part of the homological equation, the normal part corresponding on the contrary to the projection on the slave modes. 
As shown in 28], the solution to the normal part of the homological equations is uniquely defined while, on the other hand, the tangent part is underdetermined. Consequently, an infinite number of solutions exist, which gives rise to the different styles of parametrisation.

Following the general procedure shown in [35, it is expedient to define the following quantities:

$$
\theta_{r \mathcal{I}}^{(p)} \triangleq \mathbf{X}_{r}^{\mathrm{T}}\left[\begin{array}{cc}
\mathbf{M} & \mathbf{0} \\
\mathbf{0} & \mathbf{M}
\end{array}\right]\left[\begin{array}{l}
\mathbf{\Upsilon}_{\mathcal{I}}^{(p)} \\
\mathbf{\Psi}_{\mathcal{I}}^{(p)}
\end{array}\right], \quad g_{r \mathcal{I}}^{(p)} \triangleq \mathbf{X}_{r}^{\mathrm{T}}\left[\begin{array}{c}
-\mathbf{M} \boldsymbol{\nu}_{\mathcal{I}}^{(p)}-\mathbf{G}_{\mathcal{I}}^{(p)}-\mathbf{H}_{\mathcal{I}}^{(p)} \\
-\mathbf{M} \boldsymbol{\mu}_{\mathcal{I}}^{(p)}
\end{array}\right]
$$

where $\mathbf{X}_{r}$ is a master left eigenvector of the first-order problem as defined in Eq. (22), with $r \in[1, \mathrm{n}]$. In the master modal space, the tangent part then simply writes as:

$$
\left(\sigma_{\mathcal{I}}-\lambda_{r}\right) \theta_{r \mathcal{I}}^{(p)}+f_{r \mathcal{I}}^{(p)}=g_{r \mathcal{I}}^{(p)}
$$

In this expression, the unknowns are $\theta_{r \mathcal{I}}^{(p)}$, which are related to the coefficients of the nonlinear mappings, and $f_{r \mathcal{I}}^{(p)}$, related to the reduced dynamics. Eq. 411 introduces the notion of resonance, which is a cornerstone in dynamical system theory [57,58. A resonance is met when the following condition is fulfilled:

$$
\sigma_{\mathcal{I}} \approx \lambda_{r}
$$

In particular, one can observe that in this case $\theta_{r \mathcal{I}}^{(p)}$ cannot be solved for, and the only possible solution is $f_{r \mathcal{I}}^{(p)}=g_{r \mathcal{I}}^{(p)}$. Resonances and their importance in vibration theory has been analysed in many contributions and the reader is invited to refer to [13, 54, 29,5,35] for further discussions, which in particular underline the link between resonance and hardening/softening behaviour of nonlinear oscillators.

At this point the different styles of parametrisation adopt different strategies. The first strategy, called the graph style 28, aims at making the mapping as simple as possible, such that all the complexity is retained in the reduced dynamics. This leads to select, for each monomial of the tangent homological equation, $\theta_{r \mathcal{I}}^{(p)}=0$, and $f_{r \mathcal{I}}^{(p)}=g_{r \mathcal{I}}^{(p)}$. One can observe that this choice allows one to ignore any potential occurrence of resonances. Also, it leads to a simple linear relationship, in the modal space, between the normal coordinates and the master modal coordinates. As underlined in [5,35, this choice is thus equivalent to computing the invariant manifold with the center manifold technique, as proposed by Shaw and Pierre in their work on NNMs [10. The second strategy, called the normal form style, takes the opposite point of view to solve Eq. (41), and proposes to take the reduced dynamics as simple as possible, putting all the complexity in the nonlinear mappings. In this case, one then needs to distinguish between resonant and non-resonant monomials, according to Eq. 42). A non-resonant monomial can be solved setting $f_{r \mathcal{I}}^{(p)}=0$, and $\theta_{r \mathcal{I}}=g_{r \mathcal{I}}^{(p)} /\left(\sigma_{\mathcal{I}}-\lambda_{r}\right)$. On the other hand when a resonant monomial is present, then the same choice as for the graph style is retained.

In vibration theory, since the starting point is a second-order problem in time with real coordinates, it is also interesting to try to keep real quantities as far as possible in the developments in order to be able to come back to oscillator-like equations. This leads to define a full real normal form as in [13, as well as to distinguish two different normal form styles: the complex and the real one [35], the difference between the two being detailed in 35. These features are interesting in order to better understand the link between the methods previously proposed. For example, a complex normal form is used in 59, 18, 29. On the other hand, a real normal form style is detailed in 35, 60,61, and a full real normal form in [13, 22,54 .

We now turn to the solution to the non-autonomous part of the invariance equation. In this case, the presence of the external forcing adds new frequencies to the system. As a consequence, the resonance relationship needs to be revisited to take this peculiarity into account. General solutions will be detailed and discussions on the different styles of solutions will also be given.

\subsubsection{Non-autonomous part}

This Section is devoted to solving the $\varepsilon^{1}$-invariance equation 32 at arbitrary order. In particular, we assume that the autonomous part of the problem has already been tackled and $\boldsymbol{\Psi}(\mathbf{z}), \boldsymbol{\Upsilon}(\mathbf{z})$ and $\boldsymbol{f}(\mathbf{z})$ are available. The unknowns to solve for in Eq. $(32)$ are only those related to the non-autonomous part, i.e. $\hat{\boldsymbol{\Psi}}_{j}(\mathbf{z}), \hat{\boldsymbol{\Upsilon}}_{j}(\mathbf{z})$ and $\hat{\boldsymbol{f}}_{j}(\mathbf{z})$ for each of the forcing eigenvalues $\hat{\lambda}_{j}$. In order to make the presentation and notation lighter, we thus restrict ourselves in this Section to the case of a single external excitation eigenvalue $\hat{\lambda}$ and omit the subscript $(\cdot)_{j}$ in mappings and reduced dynamics. The asymptotic expansions for the Fourier coefficients associated to the expressions given in Eq. (31) are thus written as:

$$
\hat{\boldsymbol{\Psi}}_{j}(\mathbf{z})=\hat{\boldsymbol{\Psi}}(\mathbf{z})=\sum_{p=0}^{q}[\hat{\boldsymbol{\Psi}}(\mathbf{z})]_{p}, \quad \hat{\boldsymbol{\Upsilon}}_{j}(\mathbf{z})=\hat{\boldsymbol{\Upsilon}}(\mathbf{z})=\sum_{p=0}^{q}[\hat{\boldsymbol{\Upsilon}}(\mathbf{z})]_{p}, \quad \hat{\boldsymbol{f}}_{j}(\mathbf{z})=\hat{\boldsymbol{f}}(\mathbf{z})=\sum_{p=0}^{q}[\hat{\boldsymbol{f}}(\mathbf{z})]_{p}
$$


with $q$ the maximum order of expansion of the $\varepsilon^{1}$-invariance equation. Note that the maximum order of the expansion of the non-autonomous part is intimately related to that of the autonomous one, such that one has $q<o$. The notation now strictly follows the case summarized in the previous Section, and the unknowns are expanded as:

$$
[\hat{\boldsymbol{\Psi}}(\mathbf{z})]_{p}=\sum_{\mathcal{I}} \hat{\boldsymbol{\Psi}}_{\mathcal{I}}^{(p)} \pi_{\mathcal{I}}^{(p)}, \quad[\hat{\boldsymbol{\Upsilon}}(\mathbf{z})]_{p}=\sum_{\mathcal{I}} \hat{\boldsymbol{\Upsilon}}_{\mathcal{I}}^{(p)} \pi_{\mathcal{I}}^{(p)}, \quad[\hat{\boldsymbol{f}}(\mathbf{z})]_{p}=\sum_{\mathcal{I}} \hat{\mathbf{f}}_{\mathcal{I}}^{(p)} \pi_{\mathcal{I}}^{(p)}
$$

These developments, together with the known ones related to the autonomous part, need then to be inserted in the $\varepsilon^{1}$-invariance equation $(32$, from which the homological equations for each order can be derived by identification of same powers.

Contrary to the $\varepsilon^{0}$ development, whose first-order homological equations require a dedicated treatment to impose that the parametrised manifold is identity tangent to the linear subspace, the $\varepsilon^{1}$ homological equations can be derived immediately for a generic order $p$.

The main novelty of the $\varepsilon^{1}$-invariance from a computational standpoint is the development of new terms associated to the gradients with respect to the normal coordinates. A comprehensive treatment of such terms is reported in Appendix D where their derivations in a framework compatible with algorithmic implementation is reported. We can thus write the order- $p$ homological equation for any monomial $\pi_{\mathcal{I}}^{(p)}$, that is to say for any set $\mathcal{I}$ of indices spanning all the possible monomial terms of order $p$ :

$$
\begin{aligned}
& \forall p=0, \ldots, q, \quad \forall \mathcal{I} \in \hat{\mathcal{H}}^{(p)}, \\
& \hat{\sigma}_{\mathcal{I}} \mathbf{M} \hat{\boldsymbol{\Upsilon}}_{\mathcal{I}}^{(p)}+\mathbf{C} \hat{\boldsymbol{\Upsilon}}_{\mathcal{I}}^{(p)}+\mathbf{K} \hat{\mathbf{\Psi}}_{\mathcal{I}}^{(p)}+\sum_{s=1}^{2 \mathrm{n}}\left(\lambda_{s} \hat{f}_{s}^{(p)} \mathbf{M} \boldsymbol{\phi}_{s}\right)+\mathbf{M} \hat{\boldsymbol{\nu}}_{\mathcal{I}}^{(p)}+\hat{\mathbf{G}}_{\mathcal{I}}^{(p)}+\hat{\mathbf{H}}_{\mathcal{I}}^{(p)}=\hat{\mathbf{F}}_{\mathcal{I}}^{(p)}, \\
& \hat{\sigma}_{\mathcal{I}} \mathbf{M} \hat{\mathbf{\Psi}}_{\mathcal{I}}^{(p)}-\mathbf{M} \hat{\boldsymbol{\Upsilon}}_{\mathcal{I}}^{(p)}+\sum_{s=1}^{2 \mathrm{n}}\left(\hat{f}_{s \mathcal{I}}^{(p)} \mathbf{M} \boldsymbol{\phi}_{s}\right)+\mathbf{M} \hat{\boldsymbol{\mu}}_{\mathcal{I}}^{(p)}=\mathbf{0}
\end{aligned}
$$

where all quantities not previously defined are given as:

$$
\begin{aligned}
\hat{\sigma}_{\mathcal{I}} & =\hat{\lambda}+\lambda_{i_{1}}+\lambda_{i_{2}}+\ldots+\lambda_{i_{p}}, \\
\hat{\boldsymbol{\mu}}_{\mathcal{I}}^{(p)} & =\sum_{s=1}^{2 \mathrm{n}}\left(\sum_{k=2}^{p} \sum_{l=0}^{p-k} \hat{\Psi}_{\left\{i_{1} \ldots i_{l} s i_{l+k+1} \ldots i_{p}\right\}}^{(p-k+1)} f_{s\left\{i_{l+1} \ldots i_{l+k}\right\}}^{(k)}+\sum_{k=0}^{p-1} \sum_{l=0}^{p-k} \boldsymbol{\Psi}_{\left\{i_{1} \ldots i_{l} s i_{l+k+1} \ldots i_{p}\right\}}^{(p-k+1)} \hat{f}_{s\left\{i_{l+1} \ldots i_{l+k}\right\}}^{(k)}\right), \\
\hat{\boldsymbol{\nu}}_{\mathcal{I}}^{(p)} & =\sum_{s=1}^{2 \mathrm{n}}\left(\sum_{k=2}^{p} \sum_{l=0}^{p-1} \hat{\boldsymbol{\Upsilon}}_{\left\{i_{1} \ldots i_{l} s i_{l+k+1} \ldots i_{p}\right\}}^{(p-k+1)} f_{s\left\{i_{l+1} \ldots i_{l+k}\right\}}^{(k)}+\sum_{k=0}^{p-k} \sum_{l=0}^{p} \mathbf{\Upsilon}_{\left\{i_{1} \ldots i_{l} s i_{l+k+1} \ldots i_{p}\right\}}^{(p-k+1)} \hat{f}_{s\left\{i_{l+1} \ldots i_{l+k}\right\}}^{(k)}\right), \\
\hat{\mathbf{G}}_{\mathcal{I}}^{(p)} & =2 \sum_{k=1}^{p} \boldsymbol{G}\left(\boldsymbol{\Psi}_{\left\{i_{1} \ldots i_{k}\right\}}^{(k)}, \hat{\Psi}_{\left\{i_{k+1} \ldots i_{p}\right\}}^{(p-k)}\right), \\
\hat{\mathbf{H}}_{\mathcal{I}}^{(p)} & =3 \sum_{k=1}^{p-1} \sum_{l=1}^{p-k} \boldsymbol{H}\left(\boldsymbol{\Psi}_{\left\{i_{1} \ldots i_{k}\right\}}^{(k)}, \mathbf{\Psi}_{\left\{i_{k+1} \ldots i_{k+l}\right\}}^{(l)}, \hat{\mathbf{\Psi}}_{\left\{i_{k+l+1} \ldots i_{p}\right\}}^{(p-k-l)}\right), \\
\hat{\mathbf{F}}_{\mathcal{I}}^{(0)} & =\hat{\mathbf{F}}, \quad \text { and } \hat{\mathbf{F}}_{\mathcal{I}}^{(p)}=0, \quad \forall p \geq 1 .
\end{aligned}
$$

One can notice from the last line, Eq. (46f), that the forcing terms appear only at order zero of the asymptotic expansion since the applied force is not a function of the structure configuration or velocity. Consequently, the order zero $\varepsilon^{1}$-homological equation has a peculiar formulation as compared to higher order developments, and a detailed discussion about this is reported in Section 4. Interestingly, the formulation of the problem is identical to that obtained at order $\varepsilon^{0}$ for the autonomous part. One can in particular note the expressions of the coefficients $\hat{\boldsymbol{\mu}}_{\mathcal{I}}^{(p)}$ and $\hat{\boldsymbol{\nu}}_{\mathcal{I}}^{(p)}$ and compare them with $\boldsymbol{\mu}_{\mathcal{I}}^{(p)}$ and $\boldsymbol{\nu}_{\mathcal{I}}^{(p)}$ given in Eqs. 38b)-38c). Since we are working with non-autonomous terms (highlighted with hatted variables), the new coefficients make appear the cross-products of terms from the autonomous part (solved and known, without hats) with unknowns from the non-autonomous part. As in the previous Section, the order- $p$ homological equation for the monomial $\pi_{\mathcal{I}}^{(p)}$, can also be rewritten in matrix form to yield a more compact and readable form:

$$
\begin{aligned}
& \forall \mathcal{I} \in \hat{\mathcal{H}}^{(p)}, \quad \forall p \in\{1, \ldots, q\} \\
& \left(\tilde{\sigma}_{\mathcal{I}}\left[\begin{array}{cc}
\mathbf{M} & \mathbf{0} \\
\mathbf{0} & \mathbf{M}
\end{array}\right]+\left[\begin{array}{cc}
\mathbf{C} & \mathbf{K} \\
-\mathbf{M} & \mathbf{0}
\end{array}\right]\right)\left[\begin{array}{c}
\hat{\mathbf{\Upsilon}}_{\mathcal{I}}^{(p)} \\
\hat{\mathbf{\Psi}}_{\mathcal{I}}^{(p)}
\end{array}\right]+\sum_{s=1}^{n} \hat{f}_{s \mathcal{I}}^{(p)}\left[\begin{array}{cc}
\mathbf{M} & \mathbf{0} \\
\mathbf{0} & \mathbf{M}
\end{array}\right] \mathbf{Y}_{s}=\left[\begin{array}{c}
-\mathbf{M} \hat{\boldsymbol{\nu}}_{\mathcal{I}}^{(p)}-\hat{\mathbf{G}}_{\mathcal{I}}^{(p)}-\hat{\mathbf{H}}_{\mathcal{I}}^{(p)}+\hat{\mathbf{F}}_{\mathcal{I}}^{(p)} \\
-\mathbf{M} \hat{\boldsymbol{\mu}}_{\mathcal{I}}^{(p)}
\end{array}\right] .
\end{aligned}
$$


which is very similar to Eq. (39). The right-hand side of Eq. (47) has only one additional term, which is simply related to the forcing.

At this stage and elaborating on the solution already obtained for the autonomous part, one understands that the general solution to Eqs. (47) cannot be written directly, for the same reasons explained in Section 3.2.1. In order to shed light on the solution procedure, one can again use the projection of Eqs. (47) onto the master modes to derive the tangent part of the order- $p$ homological equation. To that purpose, let us now define:

$$
\hat{\theta}_{r \mathcal{I}}^{(p)} \triangleq \mathbf{X}_{r}^{\mathrm{T}}\left[\begin{array}{cc}
\mathbf{M} & \mathbf{0} \\
\mathbf{0} & \mathbf{M}
\end{array}\right]\left[\begin{array}{c}
\hat{\mathbf{\Upsilon}}_{\mathcal{I}}^{(p)} \\
\hat{\mathbf{\Psi}}_{\mathcal{I}}^{(p)}
\end{array}\right], \quad \text { and } \quad \hat{g}_{r \mathcal{I}}^{(p)} \triangleq \mathbf{X}_{r}^{\mathrm{T}}\left[\begin{array}{c}
-\mathbf{M} \hat{\boldsymbol{\nu}}_{\mathcal{I}}^{(p)}-\hat{\mathbf{G}}_{\mathcal{I}}^{(p)}-\hat{\mathbf{H}}_{\mathcal{I}}^{(p)}+\hat{\mathbf{F}}_{\mathcal{I}}^{(p)} \\
-\mathbf{M} \hat{\boldsymbol{\mu}}_{\mathcal{I}}^{(p)}
\end{array}\right]
$$

In the master modal space, the tangent part of the order- $p$ homological equation for the non-autonomous problem simply reads:

$$
\begin{aligned}
& \forall \mathcal{I} \in \hat{\mathcal{H}}^{(p)}, \forall p \in\{0, \ldots, q\}, \forall r=1, \ldots, 2 \mathrm{n}, \\
& \left(\hat{\sigma}_{\mathcal{I}}-\lambda_{r}\right) \hat{\theta}_{r \mathcal{I}}^{(p)}+\hat{f}_{r \mathcal{I}}^{(p)}=\hat{g}_{r \mathcal{I}}^{(p)} .
\end{aligned}
$$

The main difference with respect to the autonomous development is the definition of $\hat{\sigma}_{\mathcal{I}}$ given in Eq. 46a), which now contains the external forcing eigenvalue $\hat{\lambda}$, hence explicitly depending on the external forcing frequency. This reflects the fact that the calculation treats the presence of the external forcing as an additional degree of freedom with its own frequency, thus enlarging the scope of resonance relationships. This has important consequences both on the definition of resonances and on the styles of solutions that one can adopt. The following Sections are devoted to the general solution to this problem, detailing the analysis of the resonance relationship with the external forcing included, and describing how to handle them with the different styles of parametrisation.

\subsection{Solution scheme for a direct computation and styles of parametrisation}

In this Section, the solution scheme allowing one to derive a direct computation operating from the FE degrees of freedom (dofs), and applicable to both autonomous and non-autonomous parts, is first detailed. The main idea is to rely on the fact that the structure of the order- $p$ homological equations is identical for both $\varepsilon^{0}$ and $\varepsilon^{1}$-invariance equations, as underlined in the last Section. Consequently the solution scheme proposed in [35] can be easily extended to handle the time-dependent part. First one has to solve in the modal space the problem for the tangent part which is underdetermined. Next, since we operate in the physical space, a direct approach must be devised for the different possible solutions (depending on the style selected). This is realized thanks to an augmentation trick where the non-invertible matrices appearing due to resonances are bordered with eigenvectors of the kernel, see 35 for details.

Let us first begin by using a common notation for the general structure of the order- $p$ homological equation derived for both autonomous and non-autonomous parts, Eqs. (37) and (45). Both these equations can be rewritten as:

$$
\begin{aligned}
& \forall \mathcal{I} \in \mathcal{H}^{(p)}, \quad \forall p \in\{0 \ldots q\} \\
& \stackrel{\circ}{\sigma}_{\mathcal{I}} \mathbf{M} \stackrel{\circ}{\Upsilon}_{\mathcal{I}}^{(p)}+\mathbf{C} \stackrel{\circ}{\mathcal{I}}^{(p)}+\mathbf{K} \stackrel{\circ}{\Psi}^{(p)}+\sum_{s=1}^{2 \mathrm{n}} \lambda_{s} \stackrel{\circ}{f}_{s}^{(p)} \mathbf{M} \boldsymbol{\phi}_{s}=\stackrel{\circ}{\mathbf{E}}_{\mathcal{I}}^{(p)}, \\
& \stackrel{\circ}{\sigma}_{\mathcal{I}} \mathbf{M} \stackrel{\circ}{\Psi}_{\mathcal{I}}^{(p)}-\mathbf{M} \stackrel{\Upsilon}{\mathcal{I}}_{\mathcal{I}}^{(p)}+\sum_{s=1}^{2 \mathrm{n}} \stackrel{\circ}{f}_{s \mathcal{I}}^{(p)} \mathbf{M} \boldsymbol{\phi}_{s}=-\mathbf{M} \dot{\boldsymbol{\mu}}_{\mathcal{I}}^{(p)}
\end{aligned}
$$

The notation $(\cdot)$ is here used to denote the quantities associated with either $\varepsilon^{0}$ or $\varepsilon^{1}$ developments. For instance $\stackrel{\circ}{\mathcal{I}}_{\mathcal{I}}$ refers to $\sigma_{\mathcal{I}}$ for the autonomous problem, and to $\hat{\sigma}_{\mathcal{I}}$ for the non-autonomous case. The same holds for mappings and reduced dynamics terms. The right-hand sides of Eqs. (37) and (45) have been rewritten with the simple generic term $\stackrel{\circ}{\mathbf{E}}_{\mathcal{I}}^{(p)}$, whose expression for the $\varepsilon^{0}$-invariance is given as:

$$
\stackrel{\circ}{\mathbf{I}}_{\mathcal{I}}^{(p)} \triangleq \mathbf{E}_{\mathcal{I}}^{(p)} \triangleq-\mathbf{M} \nu_{\mathcal{I}}^{(p)}-\mathbf{G}_{\mathcal{I}}^{(p)}-\mathbf{H}_{\mathcal{I}}^{(p)}
$$

while its expression for the $\varepsilon^{1}$-invariance equations is:

$$
\stackrel{\circ}{\mathbf{E}}_{\mathcal{I}}^{(p)} \triangleq \hat{\mathbf{E}}_{\mathcal{I}}^{(p)} \triangleq-\mathbf{M} \hat{\boldsymbol{\nu}}_{\mathcal{I}}^{(p)}-\hat{\mathbf{G}}_{\mathcal{I}}^{(p)}-\hat{\mathbf{H}}_{\mathcal{I}}^{(p)}+\hat{\mathbf{F}}_{\mathcal{I}}^{(p)}
$$

One important feature of Eq. (50) is that for any order there exists an algebraic relation between $\dot{\Upsilon}_{\mathcal{I}}^{(p)}$ and $\stackrel{\circ}{\Psi}_{\mathcal{I}}^{(p)}$. This implies that Eq. $(50 \mathrm{~b})$ can be exploited to halve the number of equations associated to the 
mappings. The resulting linear system obtained for $\stackrel{\circ}{\Psi}_{\mathcal{I}}^{(p)}$, after condensing the velocity mapping terms, is equal to:

$$
\left[\circ_{\mathcal{I}}^{2} \mathbf{M}+\stackrel{\circ}{\sigma}_{\mathcal{I}} \mathbf{C}+\mathbf{K}\right] \stackrel{\circ}{\Psi}_{\mathcal{I}}^{(p)}+\sum_{s=1}^{2 \mathrm{n}}\left(\circ_{\mathcal{I}}-\bar{\lambda}_{s}\right) \mathbf{M} \boldsymbol{\phi}_{s} \stackrel{\circ}{f}_{s}^{(p)}=\stackrel{\circ}{\mathbf{E}}_{\mathcal{I}}^{(p)}-\left[\circ_{\mathcal{I}} \mathbf{M}+\mathbf{C}\right] \stackrel{\circ}{\boldsymbol{\mu}}_{\mathcal{I}}^{(p)},
$$

where the following identity has been used for the derivation (see Appendix A of [35] for the proof of this property):

$$
\left(\left(\stackrel{\circ}{\sigma}_{\mathcal{I}}+\lambda_{j}\right) \mathbf{M}+\mathbf{C}\right) \boldsymbol{\phi}_{j}=\left(\stackrel{\circ}{\sigma}_{\mathcal{I}}-\bar{\lambda}_{j}\right) \mathbf{M} \boldsymbol{\phi}_{j} .
$$

In order to further unify the presentation of the solutions for both autonomous and non-autonomous part, let us also introduce a generic notation for the tangent part of the order- $p$ homological equation as:

$$
\begin{aligned}
& \forall p \in\{0, \ldots, q\}, \forall \mathcal{I} \in \stackrel{\circ}{\mathcal{H}}^{(p)}, \forall r=1, \ldots, 2 \mathrm{n}, \\
& \left(\stackrel{\circ}{\sigma} \mathcal{I}^{\left.-\lambda_{r}\right)} \dot{\circ}_{r \mathcal{I}}^{(p)}+\stackrel{\circ}{f}_{r \mathcal{I}}^{(p)}=\stackrel{\circ}{g}_{r}^{(p)},\right.
\end{aligned}
$$

which holds for both Eq. (41) (by simply omitting the upper circle) and Eq. 49) (by replacing the circle with a hat). As explained in Section 3.2.1 the two basic solutions to Eq. (55) are either to set $\stackrel{\circ}{r \mathcal{I}}(p)^{(p)} 0$ and

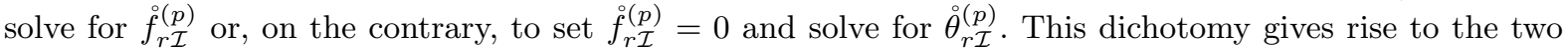
main styles of parametrisation, the graph style and the normal form style. Unfortunately, while the writing of these two types of solutions is obvious in the modal space, it is not in the physical space where all the coordinates are interwoven. Moreover, one needs to take care of the occurrence of resonances, since in this case the first term in Eq. (55) vanishes and the dichotomy is not applicable anymore.

In order to simultaneously write the solutions in physical space for a direct computation, and take care of the resonances, the set $\mathcal{R}$ has been introduced in [35] as the set of indices such that the choice of setting $\stackrel{\circ}{\theta}_{r \mathcal{I}}^{(p)}=0$ is made. Following a direct parametrisation approach, imposing $\stackrel{\circ}{r \mathcal{I}}(p)^{(p)} 0$ is equivalent to enforce orthogonality between the associated mappings $\left(\dot{\boldsymbol{\Psi}}_{\mathcal{I}}^{(p)}, \stackrel{\circ}{\mathbf{I}}_{\mathcal{I}}^{(p)}\right)$, and the left eigenfunction $\mathbf{X}_{r}$ associated to the relevant resonant monomial, which writes (see [35] for details):

$$
\mathbf{X}_{r}^{\mathrm{T}}\left[\begin{array}{cc}
\mathbf{M} & \mathbf{0} \\
\mathbf{0} & \mathbf{M}
\end{array}\right]\left[\begin{array}{c}
\stackrel{\circ}{\mathbf{T}}_{\mathcal{I}}^{(p)} \\
\stackrel{\circ}{\Psi}_{\mathcal{I}}^{(p)}
\end{array}\right]=0
$$

or equivalently:

$$
\boldsymbol{\phi}_{r}^{\mathrm{T}} \mathbf{M} \dot{\Upsilon}_{\mathcal{I}}^{(p)}=\bar{\lambda}_{r} \boldsymbol{\phi}_{r}^{\mathrm{T}} \mathbf{M} \stackrel{\circ}{\Psi}_{\mathcal{I}}^{(p)}
$$

Using Eq. 50b in order to eliminate $\stackrel{\Upsilon}{\Upsilon}_{\mathcal{I}}^{(p)}$, from the above equation, one can finally rewrite the orthogonality condition (57) as:

$$
\boldsymbol{\phi}_{r}^{\mathrm{T}} \mathbf{M}\left(\stackrel{\circ}{\sigma}_{\mathcal{I}} \stackrel{\circ}{\Psi}_{\mathcal{I}}^{(p)}+\sum_{s=1}^{2 \mathrm{n}}\left(\phi_{s} \stackrel{\circ}{s}_{s \mathcal{I}}^{(p)}\right)+\stackrel{\circ}{\boldsymbol{\mu}}_{\mathcal{I}}^{(p)}\right)-\bar{\lambda}_{r} \boldsymbol{\phi}_{r}^{\mathrm{T}} \mathbf{M} \stackrel{\circ}{\Psi}_{\mathcal{I}}^{(p)}=0,
$$

This last equation will be used in the forthcoming developments to augment the system and write the direct solution from the physical space.

Remembering that the order- $p$ homological equation is written for each set $\mathcal{I}$ defining a monomial, and the definition of $\mathcal{R}$ just recalled, one can now rewrite the general solutions in the modal space as:

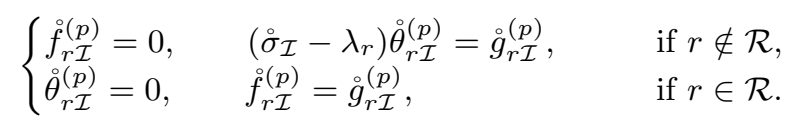

Three styles of parametrisations have been defined in 35], and their difference can be highlighted by focusing on how they fill the set $\mathcal{R}$. The graph style, irrespective of resonance conditions, always sets

$$
\mathcal{R}_{\text {Graph }}=\{12 \ldots 2 \mathrm{n}\} \text {. }
$$

On the contrary, the complex normal form style $(\mathrm{CNF})$ is defined via

$$
\mathcal{R}_{\mathrm{CNF}}=\{r\}, \quad \forall \mathcal{I}, r: \stackrel{\circ}{\sigma}_{\mathcal{I}} \approx \lambda_{r} .
$$

while, finally, the real normal form style (RNF) is such that

$$
\mathcal{R}_{\mathrm{RNF}}=\left\{r r^{*}\right\}, \quad \forall \mathcal{I}, r, r^{*}: \stackrel{\circ}{\sigma}_{\mathcal{I}}^{2} \approx \lambda_{r}^{2} \approx \lambda_{r^{*}}^{2},
$$

where $r^{*}$ is defined as

$$
r^{*}= \begin{cases}r+\mathrm{n} & \text { if } r \leq \mathrm{n} \\ r-\mathrm{n} & \text { if } r>\mathrm{n}\end{cases}
$$


The overall linear system that yields the displacement mappings and the reduced dynamic coefficients associated to a given monomial is of size $\mathrm{N}+m, m$ being the number of resonant degrees of freedom, i.e. the cardinality of $\mathcal{R}$. The resulting matrix structure is reported in [35] for the autonomous case, which extends exactly to the non-autonomous system since the problems have the same shape. For the sake of brevity, the augmentation strategy using a bordering technique is not repeated here, and the interested reader can find the details of this procedure in [35]. Only the results are reported, for completeness.

For the complex normal form $(\mathrm{CNF})$, where the size of the set $\mathcal{R}$ is the smallest one, the augmentation procedure leads to solve the following problem:

$$
\left[\begin{array}{cc}
\stackrel{\circ}{\sigma}_{\mathcal{I}}^{2} \mathbf{M}+\stackrel{\circ}{\sigma}_{\mathcal{I}} \mathbf{C}+\mathbf{K} & \left(\stackrel{\circ}{\sigma}_{\mathcal{I}}-\bar{\lambda}_{r}\right) \mathbf{M} \boldsymbol{\phi}_{r} \\
\left(\circ_{\mathcal{I}}-\bar{\lambda}_{r}\right) \boldsymbol{\phi}_{r}^{\mathrm{T}} \mathbf{M} & 1
\end{array}\right]\left[\begin{array}{c}
\stackrel{\circ}{\mathbf{\Psi}}_{\mathcal{I}}^{(p)} \\
\circ_{r}^{(p)}
\end{array}\right]=\left[\begin{array}{c}
\stackrel{\circ}{\mathbf{E}}_{\mathcal{I}}^{(p)}-\left[\stackrel{\circ}{\mathcal{I}}_{\mathcal{I}} \mathbf{M}+\mathbf{C}\right] \stackrel{\circ}{\mathcal{M}}_{\mathcal{I}}^{(p)} \\
-\boldsymbol{\phi}_{r}^{\mathrm{T}} \mathbf{M} \stackrel{\circ}{\boldsymbol{\mu}}_{\mathcal{I}}^{(p)}
\end{array}\right]
$$

The added line corresponds to rewriting the orthogonality condition given by Eq. (58) in matrix form, using the fact that in this case the cardinality of $\mathcal{R}$ is one.

For the real normal form style (RNF) the size of $\mathcal{R}$ is doubled, which leads to the following system:

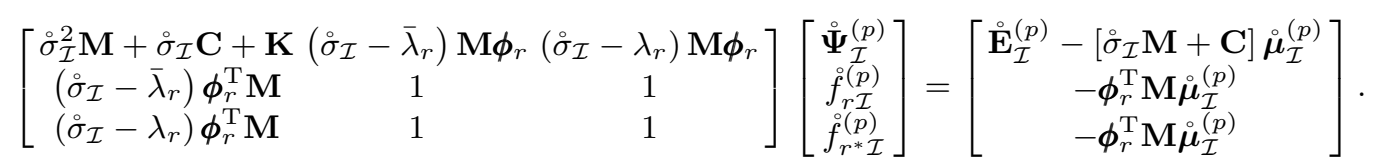

In this case the two added lines deduces from $\mathcal{R}_{\mathrm{RNF}}=\left\{r, r^{*}\right\}$, and each of them corresponds to Eq. (58).

Finally, the graph style parametrisation is characterized by a complete set $\mathcal{R}$ containing the coordinates of all master modes; consequently the size of the system to solve is larger and reads:

$$
\left[\begin{array}{cc}
\stackrel{\circ}{\sigma}_{\mathcal{I}}^{2} \mathbf{M}+\stackrel{\circ}{\sigma}_{\mathcal{I}} \mathbf{C}+\mathbf{K} & \mathbf{M} \boldsymbol{\phi}\left(\mathbf{I}_{[2 \mathrm{n}]} \stackrel{\circ}{\mathcal{I}}_{\mathcal{I}}-\overline{\boldsymbol{\lambda}}\right) \\
\left(\mathbf{M} \boldsymbol{\phi}\left(\mathbf{I}_{[2 \mathrm{n}]} \stackrel{\circ}{\mathcal{I}}_{\mathcal{I}}-\overline{\boldsymbol{\lambda}}\right)\right)^{\mathrm{T}} & {\left[\begin{array}{l}
\mathbf{I}_{[\mathrm{n}]} \mathbf{I}_{[\mathrm{n}]} \\
\mathbf{I}_{[\mathrm{n}]} \mathbf{I}_{[\mathrm{n}]}
\end{array}\right]}
\end{array}\right]\left[\begin{array}{l}
\stackrel{\circ}{\mathbf{\Psi}}_{\mathcal{I}}^{(p)} \\
\stackrel{\mathbf{f}}{\mathcal{I}}_{(p)}^{(p)}
\end{array}\right]=\left[\begin{array}{c}
\stackrel{\circ}{\mathbf{E}}_{\mathcal{I}}^{(p)}-\left[\circ_{\mathcal{I}} \mathbf{M}+\mathbf{C}\right] \stackrel{\circ}{\boldsymbol{\mu}}_{\mathcal{I}}^{(p)} \\
-\boldsymbol{\phi}^{\mathrm{T}} \mathbf{M} \stackrel{\circ}{\mathcal{I}}_{\mathcal{I}}^{(p)}
\end{array}\right],
$$

where $\mathbf{I}_{[\mathrm{n}]}$ refers to the identity matrix of size $\mathrm{n} \times \mathrm{n}$. All linear systems reported in this Section are sparse and can be generated using the mass, damping, and stiffness matrices as well as standard functions to compute system nonlinearities over the domain. Importantly, all the linear problems involve invertible matrices that have been built based on the knowledge of the solution in the modal space. Singularities due to the occurrence of resonances have been treated in each system by bordering the singular parts with the eigenvectors of the kernels.

\section{Single master mode analysis and resonances}

In this Section, the new resonances appearing in the $\varepsilon^{1}$-invariance equations, shown in Eq. (49) and briefly underlined at the end of Section 3.2.2 are further analyzed together with their consequences on the reduced dynamics and the nonlinear mappings. Importantly, the emphasis is put on the appearance of new trivial resonance relationships in the non-autonomous part that are completely related to the fact that the forcing is now taken into account. Following [13,54, resonances are named trivial when they cannot be avoided, due to the peculiar structure of the eigenspectrum of vibration problems, composed of complex conjugate pairs. While these resonances have been already well analyzed for the autonomous problem, this Section tackles their definition and appearance in the context of a forced system. The consequences of these trivial resonance relationships are numerous and important.

In order to initiate the analysis, we first restrict ourselves to the case where reduction to a single master mode is undertaken, with the assumption of a single excitation frequency $\Omega$, with associated eigenvalues $\hat{\lambda}_{j}=\hat{\lambda}_{+}=+\mathfrak{i} \Omega$ and $\hat{\lambda}_{j+\hat{n}}=\hat{\lambda}_{-}=-\mathfrak{i} \Omega$, which is consistent with the definitions given in Section 2.2 In particular, it is assumed that the master mode does not generate any internal resonance with any of the slave modes, ensuring that reduction to single mode is valid. In accordance with the notation introduced before Eq. (22), the label of the master mode should be $m_{1}$. However, for the sake of keeping the expressions as simple as possible, we will use the label $m$ and hence the selected master mode is denoted $\boldsymbol{\Phi}_{m}$, with eigenfrequency $\omega_{m}$ and complex eigenvalue $\Lambda_{m}$. With a single mode assumption, the normal variables list has two entries that are indexed with 1 and $2=1^{*}: \mathbf{z}=\left(z_{1}, z_{2}\right)=\left(z_{1}, z_{1^{*}}\right)$. According to the conventions set in Eq. 22, $\lambda_{1}=\Lambda_{m}, \lambda_{1^{*}}=\bar{\Lambda}_{m}$, and $\phi_{1}=\phi_{1^{*}}=\boldsymbol{\Phi}_{m}$.

At order $p$ of the development the resonance relationships, stemming from the non-autonomous part with the single master mode assumption, simply read $\hat{\sigma}_{ \pm, \mathcal{I}} \approx \lambda_{1}$ or $\hat{\sigma}_{ \pm, \mathcal{I}} \approx \bar{\lambda}_{1}$, where $\hat{\sigma}_{ \pm, \mathcal{I}}$ refers to the 
two possible cases:

$$
\begin{aligned}
& \hat{\sigma}_{+, \mathcal{I}}=\hat{\lambda}_{+}+\lambda_{i_{1}}+\lambda_{i_{2}}+\ldots+\lambda_{i_{p}}=+\mathfrak{i} \Omega+\lambda_{i_{1}}+\lambda_{i_{2}}+\ldots+\lambda_{i_{p}}, \\
& \hat{\sigma}_{-, \mathcal{I}}=\hat{\lambda}_{-}+\lambda_{i_{1}}+\lambda_{i_{2}}+\ldots+\lambda_{i_{p}}=-\mathfrak{i} \Omega+\lambda_{i_{1}}+\lambda_{i_{2}}+\ldots+\lambda_{i_{p}},
\end{aligned}
$$

Let us recall that this condition is associated to the monomial $\pi_{\mathcal{I}}^{(p)}=z_{i_{1}} z_{i_{2}} \ldots z_{i_{p}}$ where $\mathcal{I}=\left\{i_{1} i_{2} \ldots i_{p}\right\}$ is a set of indices spanning master modes only. As a consequence, in the present case the indices will be either 1 or $1^{*}$ only, where the conjugate notation introduced in Eq. 63 is used. Finally, since we are assuming small damping, the resonance condition is to be checked on the imaginary parts of the eigenvalues, in order to avoid the appearance of small denominators. Under this assumption, whenever both 1 and $1^{*}$ are present in $\mathcal{I}$, then the associated summation in the resonance relationship vanishes since $\lambda_{1}+\lambda_{1^{*}} \approx i \omega_{m}-i \omega_{m}=0$. One can thus simply discard all pairs $\left(1,1^{*}\right)$ in the set $\mathcal{I}$ and simply rewrite (67) as

$$
\hat{\sigma}_{ \pm, \mathcal{I}}=\hat{\lambda}_{ \pm}+k \lambda_{1}, \quad \text { or } \quad \hat{\sigma}_{ \pm, \mathcal{I}}=\hat{\lambda}_{ \pm}+k \lambda_{1^{*}},
$$

where the integer $k \geq 0$ has been defined as the number of occurrences of index 1 (resp. $1^{*}$ index) in $\mathcal{I}$ that are not paired with their conjugate. Note in particular that when the order $p$ is even (resp. odd), then $k$ is necessarily an even (resp. odd) number smaller or equal to $p$. For example when $p=3$, then one can create $\hat{\sigma}_{ \pm, \mathcal{I}}=\hat{\lambda}_{ \pm}+\lambda_{1}$, if $\mathcal{I}=\left\{\begin{array}{ll}1 & 1^{*} \\ 1\end{array}\right\}, \hat{\sigma}_{ \pm, \mathcal{I}}=\hat{\lambda}_{ \pm}+3 \lambda_{1}$, if $\mathcal{I}=\left\{\begin{array}{lll}1 & 1 & 1\end{array}\right\}$, and similarly $\hat{\sigma}_{ \pm, \mathcal{I}}=\hat{\lambda}_{ \pm}+\lambda_{1^{*}}$, or $\hat{\sigma}_{ \pm, \mathcal{I}}=\hat{\lambda}_{ \pm}+3 \lambda_{1^{*}}$, with the conjugate $\mathcal{I}$ sets.

A resonance condition is met when $\hat{\sigma}_{ \pm, \mathcal{I}}$ is close to the eigenvalue of the master mode, i.e. when $\hat{\sigma}_{ \pm, \mathcal{I}} \approx \lambda_{1}$ or $\hat{\sigma}_{ \pm, \mathcal{I}} \approx \lambda_{1^{*}}$. Inspecting the four possible cases arising from these relationships, it can be shown that all of them can be recast in the compact condition $\pm \Omega \approx( \pm 1 \pm k) \omega_{m}$. Excluding the case $\Omega \approx 0$, not considered here since static forcing with vanishing frequencies are not taken into account, one can see that they all reduce to the simple condition:

$$
\Omega \approx \gamma \omega_{m}
$$

with $\gamma \geq 1$ an integer such that

$$
\gamma=k-1, \quad \text { or } \quad \gamma=k+1 .
$$

As a summary, trivial resonance relationships appear when the forcing frequency is equal to a multiple value of the eigenfrequency of the master mode. Also, as a consequence of Eq. (70), whenever the forcing frequency is an odd (resp. even) multiple of the resonance frequency, i.e. $\gamma$ is odd (resp. even), then trivial resonances are met only at even (resp. odd) orders of the expansion.

As a first conclusion, the forcing frequency needs to be an integer multiple of the master mode's eigenfrequency to create new trivial resonances. If this is met, then the condition provided by Eq. 70 has to be checked at any order $p$ of the development and resonant monomials need to be embedded in the reduced dynamics. Numerous different cases need then to be separately analyzed for increasing values of $\gamma$, and for each of them important physical features arise. The analysis proposed here does not aim at being complete for any $\gamma$ and we will only propose, in the following Sections, a first analysis of the basic cases appearing for small values of $\gamma$. To begin with, the simplest case of non-resonant forcing, where the excitation frequency is different from all possible multiples of the master mode's eigenfrequency, is detailed.

\subsection{The case of a non-resonant forcing}

In this case the relationship $\Omega=\gamma \omega_{m}$ is not verified whatever the value of $\gamma$, which means that the forcing frequency is far from any multiple value of the master mode's eigenfrequency. The main consequence is that, at any order $p$ of the development, no extra trivial resonance conditions will appear in the processing of the non-autonomous part. Returning back to Eq. (55) which is written for both autonomous and nonautonomous parts, one can thus immediately conclude that the simplest choice for the solution in the tangent space is to cancel all the terms of the reduced dynamics, at each order: $\hat{f}_{r \mathcal{I}}^{(p)}=0$. This choice is naturally associated with a complex normal form style parametrisation. Let us thus assume that this parametrisation style has been selected for the remainder of the discussion, since it allows a more direct understanding. The cases of other parametrisations styles (graph or real normal form), will be commented at the end of the Section. Putting back this result in the homological equations at order $p$, Eq. (53), shows that the remaining unknowns, which are the coefficients of the nonlinear mappings, can be computed from:

$$
\left(\hat{\sigma}_{ \pm, \mathcal{I}}^{2} \mathbf{M}+\hat{\sigma}_{ \pm, \mathcal{I}} \mathbf{C}+\mathbf{K}\right) \hat{\mathbf{\Psi}}_{ \pm}^{(p)}=\hat{\mathbf{E}}_{ \pm, \mathcal{I}}^{(p)}-\left[\hat{\sigma}_{ \pm, \mathcal{I}} \mathbf{M}+\mathbf{C}\right] \hat{\boldsymbol{\mu}}_{ \pm, \mathcal{I}}^{(p)}
$$


At order zero, a simple and exact solution for the mapping $\hat{\boldsymbol{\Psi}}_{ \pm}^{(0)}$ is found by replacing $\hat{\mathbf{E}}_{ \pm, \mathcal{I}}^{(0)}$ by its value given in Eq. (52). All the right-hand side terms are vanishing, except for the external forcing term, leading to:

$$
\left(\hat{\lambda}_{ \pm}^{2} \mathbf{M}+\hat{\lambda}_{ \pm} \mathbf{C}+\mathbf{K}\right) \hat{\mathbf{\Psi}}_{ \pm}^{(0)}=\hat{\mathbf{F}}_{ \pm}
$$

From this last equation, one immediately understands that the zero-order solution to the non-autonomous part of the mapping $\hat{\boldsymbol{\Psi}}_{+}^{(0)}$ is given by the linear transfer function of the underlying linear oscillatory problem, which gives a very simple physical understanding. Furthermore, since no resonances are verified, we have that $\hat{\boldsymbol{f}}=\mathbf{0}$ and the reduced dynamics is simply conveyed by the autonomous part, regardless of the order of the asymptotic expansion:

$$
\dot{\mathbf{z}}=f(\mathbf{z}) .
$$

Since the dynamics is assumed to be lightly damped, with $\operatorname{Re}\left[\lambda_{j}\right]<0$ for all modes, the only steady-state solution to the reduced dynamics is thus the origin $\mathbf{z}=\mathbf{0}$. Hence in this case of non-resonant forcing, the reduced dynamics is trivial and leads to the solution at rest. An important consequence is that all the terms of the mappings for the autonomous part, $\Psi^{(p)}(\mathbf{z})$, vanish and are thus not needed to compute the displacement field of the system (since being at least proportional to $\mathbf{z}$, see their definition in Eq. (36)). The same applies for all terms of the non-autonomous mappings $\hat{\boldsymbol{\Psi}}_{ \pm}^{(p)}$ for orders $p \geq 1$. Under the assumption of single excitation frequency, the only remaining term in all the calculations are those from the zero-order mapping of the non-autonomous part, so that the only admissible steady state periodic motion of the system reads:

$$
\mathbf{U}=\varepsilon\left(\hat{\mathbf{\Psi}}_{+}^{(0)} e^{+\mathrm{i} \Omega t}+\hat{\mathbf{\Psi}}_{-}^{(0)} e^{-\mathrm{i} \Omega t}\right)
$$

with $\hat{\Psi}_{ \pm}^{(0)}$ given by 72 . These results show that in this case of out-of-resonance excitation, the nonlinearities can be fully neglected, which is coherent with the underlying assumptions since the forcing is assumed to be small, such that when operated out of any multiple of the eigenfrequency of the master mode, it cannot create large amplitude responses.

The effect of the external forcing on the computed whisker is better highlighted by projecting the problem in the modal space, in order to separate the respective effects on the master mode, and on a generic slave mode. Let us define the mass-weighted projection of a mapping vector onto a mode as:

$$
\stackrel{\circ}{\Psi}_{i \mathcal{I}}^{(p)}=\boldsymbol{\Phi}_{i}^{\mathrm{T}} \mathbf{M} \dot{\circ}_{\mathcal{I}}^{(p)}, \quad \stackrel{\circ}{i \mathcal{I}}^{(p)}=\boldsymbol{\Phi}_{i}^{\mathrm{T}} \mathbf{M} \stackrel{\Upsilon}{\Upsilon}_{\mathcal{I}}^{(p)}
$$

We can then compute modal displacements and velocities through mass-weighted projection of displacement $\mathbf{U}$ and velocity $\mathbf{V}$ along any eigenmode $\boldsymbol{\Phi}_{i}$ :

$$
\begin{aligned}
& \mathrm{u}_{i}=\boldsymbol{\Phi}_{i}^{\mathrm{T}} \mathbf{M U}=\varepsilon\left(\hat{\Psi}_{i+}^{(0)} e^{+\mathrm{i} \Omega t}+\hat{\Psi}_{i-}^{(0)} e^{-\mathrm{i} \Omega t}\right), \\
& \mathrm{v}_{i}=\boldsymbol{\Phi}_{i}^{\mathrm{T}} \mathbf{M} \mathbf{V}=\varepsilon\left(\hat{\Upsilon}_{i+}^{(0)} e^{+\mathrm{i} \Omega t}+\hat{\Upsilon}_{i-}^{(0)} e^{-\mathrm{i} \Omega t}\right)=\varepsilon \mathrm{i} \Omega\left(\hat{\Psi}_{i+}^{(0)} e^{+\mathrm{i} \Omega t}-\hat{\Psi}_{i-}^{(0)} e^{-\mathrm{i} \Omega t}\right),
\end{aligned}
$$

where the specific solution for this non-resonant case, Eq. (74), has been used; subscripts \pm refer to the forcing frequencies $\pm \mathfrak{i} \Omega$, and the following relation between zero-order non-autonomous mappings, stemming from Eq. (50b), has been used:

$$
\hat{\boldsymbol{\Upsilon}}_{+}^{(0)}=+\mathfrak{i} \Omega \hat{\Psi}_{+}^{(0)}, \quad \text { and } \quad \hat{\boldsymbol{\Upsilon}}_{-}^{(0)}=-\mathfrak{i} \Omega \hat{\Psi}_{-}^{(0)}
$$

Equation (76) allows one to better understand and represent the motions of the computed whisker in phase space. At order zero, the effect of a non-resonant forcing is to introduce a time-modulation of the invariant set. Indeed, all modal coordinates undergo harmonic motion with displacement and velocity having a 90 degrees phase difference, as given by Eq. 777). Consequently, whenever the projection of the zero-order mapping is non-zero for a given mode, then the manifold exhibits a rigid rotation along the associated modal plane in phase space.

In order to better highlight the physical implications of zero-order terms in the non-resonant forcing scenario, Fig. 2 shows two schematic representations of the whisker computed for a system subjected to single harmonic excitation, represented along the $\mathrm{u}_{m}, \mathrm{v}_{m}$, and $\mathrm{u}_{s}$ coordinates, where the subscript $m$ stands for the master mode, and $s$ stands for a generic slave mode. Figure 2(a) represents the condition such that $\hat{\Psi}_{m \pm}^{(0)} \neq 0$ (which in turn implies $\hat{\Upsilon}_{m \pm}^{(0)} \neq 0$ thanks to Eq. 777 ), and $\hat{\Psi}_{s \pm}^{(0)}=0$. This condition is met when the projection of the zero-order mapping onto any slave coordinate is zero. One can verify which forcing conditions yields such behaviour, by taking the projection of Eq. 72 onto a generic mode $\boldsymbol{\Phi}_{i}$ :

$$
\left(\hat{\sigma}_{ \pm, \mathcal{I}}^{2}+2 \xi_{i} \omega_{i} \hat{\sigma}_{ \pm, \mathcal{I}}+\omega_{i}^{2}\right) \hat{\Psi}_{i \pm}^{(0)}=\boldsymbol{\Phi}_{i}^{\mathrm{T}} \hat{\mathbf{F}}_{ \pm}
$$



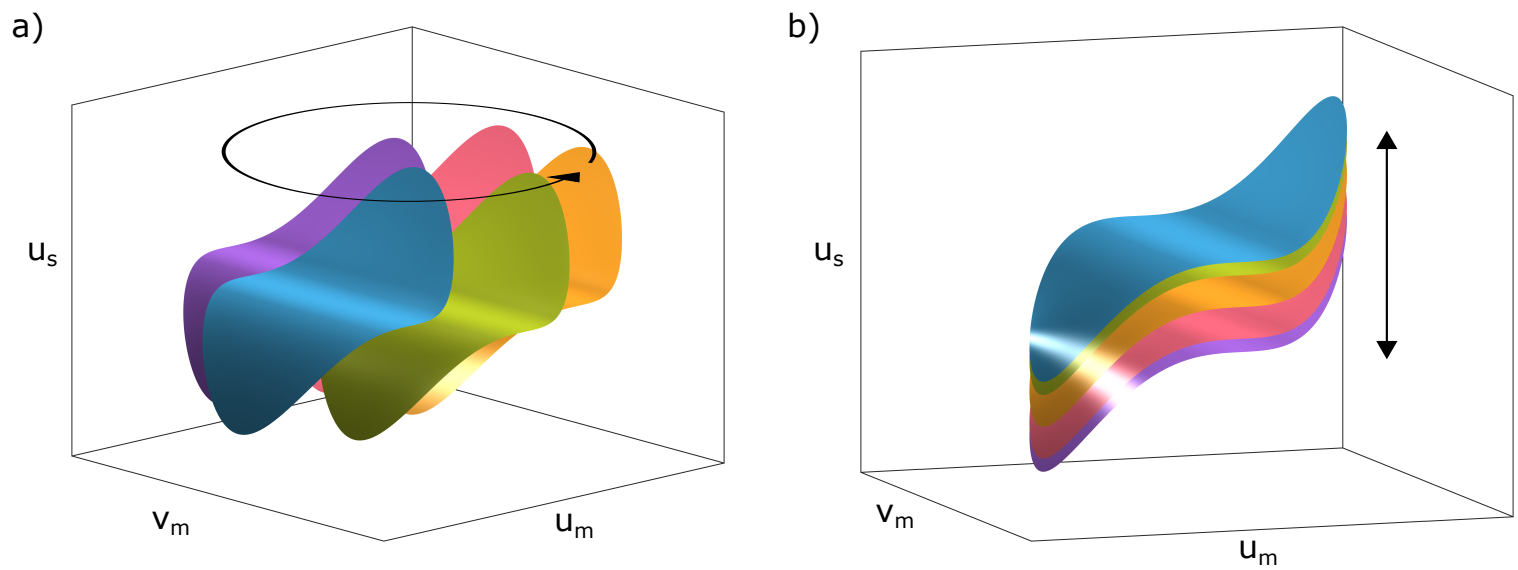

Fig. 2 Whisker motion along time when the non-autonomous mapping is truncated at order zero. (a) whisker subjected to $\hat{\Psi}_{m \pm}^{(0)} \neq 0$, and $\hat{\Psi}_{s \pm}^{(0)}=0$. (b) whisker subjected to $\hat{\Psi}_{m \pm}^{(0)}=0$ and $\hat{\Psi}_{s \pm}^{(0)} \neq 0$. The two pictures highlight the effects conveyed by non-autonomous mappings colinear with the master mode and by mappings colinear with a slave mode. In (a) colors $(\square),(\square),(\square),(\square),(\square)$ correspond to a phase $\Theta=\Omega t$ equal to $0,2 \pi / 5,4 \pi / 5,6 \pi / 5$, and $8 \pi / 5$, respectively. In (b) colors $(\square),(\square),(\square),(\square),(\square)$ correspond to a phase $\Theta=\Omega t$ equal to $0, \pi / 4, \pi / 2,3 \pi / 4$, and $\pi$, respectively

which highlights that $\hat{\Psi}_{i \pm}^{(0)} \neq 0$ whenever the modal participation factor of the force along a given linear mode is not zero. Therefore, Fig. 2 a represents the condition such that the force is perfectly co-linear with the master mode $\boldsymbol{\Phi}_{m}$, a loading condition that has been exploited for instance in 33. 35 .

A different scenario is reported in Fig. 2(b), which shows a whisker exhibiting zero-order motion along $\mathrm{u}_{s}$ only. In the selected phase space $\left(\mathrm{u}_{m}, \mathrm{v}_{m}, \mathrm{u}_{s}\right)$, this represents a rigid oscillatory motion along $\mathrm{u}_{s}$ which is the result of a circular motion of the invariant set along the $\left(\mathrm{u}_{s}, \mathrm{v}_{s}\right)$ plane as given by Eq. (77). Under the assumption of non-resonant forcing, such kind of response is met when $\boldsymbol{\Phi}_{s}^{\mathrm{T}} \hat{\mathbf{F}}_{ \pm} \neq 0$, and the force is perfectly orthogonal to the master subspace.

Two last remarks are worth stressing. Charts reported in Fig. 2 represent the two opposite loading conditions for a system parametrised along a given master mode $\boldsymbol{\Phi}_{m}$, i.e. a whisker obtained for a system subjected to a force that is co-linear with $\boldsymbol{\Phi}_{m}$, and a whisker derived for a system excited with a force that is orthogonal to the master mode. In general, the applied forcing is neither completely orthogonal nor exactly co-linear with the given master subspace. As a consequence, the parametrised invariant manifold exhibits zero-order modulations that are a combination of those depicted in Fig. 2, i.e. the system undergoes harmonic motion both along the plane spanned by $\boldsymbol{\Phi}_{m}$ and along any plane associated to a slave mode $\boldsymbol{\Phi}_{s}$. Furthermore, the presented treatment is proposed under the assumption that the system is excited out of resonance and that the parametrisation style is either CNF or RNF. On the other hand, if a graph style parametrisation is selected, one has then $\stackrel{\circ}{\mathcal{I}}_{\mathcal{I}}^{(p)}=0$. Consequently $\boldsymbol{\Phi}_{m}^{\mathrm{T}} \hat{\mathbf{\Psi}}_{ \pm}^{(0)}=0$, hence modulations of the whisker in time along the master mode directions are not possible, and the main oscillations in such case are represented by Fig. 2(b).

Contrary to order zero non-autonomous mappings, higher order terms yield a whisker that undergoes time-dependent distortions, in addition to the main oscillatory motion conveyed by order 0 . These effects are hardly observed in structures of practical interest, yet they can be found in presence of strong nonlinear coupling between master and slave modes and forcing on a slave mode. Examples of such modulations are highlighted in Section 6 .

\subsection{System driven at main resonance: primary resonance and isolated solutions}

Let us begin the analysis of the resonance condition $\Omega \approx \gamma \omega_{m}$ with the case $\gamma=1$. Recalling that these resonance conditions appear from the order $\gamma-1$ of the asymptotic development, the consequence will thus develop from the zero-order term of the non-autonomous part. Since now $\Omega \approx \omega_{m}$, this case is called main resonant excitation. As it is hereafter discussed, this condition yields two different cases according to the force distribution acting on the body. One is the primary resonance, which is more familiar in the mechanical context and is observed when the master modal force is not zero. On the other hand, a different case is exhibited when the forcing acts on coupled slave modes, and is thus perfectly orthogonal to the master one, which in turn yields conditions for isolated solutions. 
To give a physical explanation, let us first restrict ourselves to the case of a graph style or a real normal form style parametrisation, since the consequences are easier to understand. The case of the complex normal form style will be commented later. Focusing on the zero-order terms of the non-autonomous part, the associated homological equation can be rewritten from Eq. (53) as:

$$
\left(\hat{\lambda}_{ \pm}^{2} \mathbf{M}+\hat{\lambda}_{ \pm} \mathbf{C}+\mathbf{K}\right) \hat{\mathbf{\Psi}}_{ \pm}^{(0)}+\left(\hat{\lambda}_{ \pm}-\lambda_{1^{*}}\right) \mathbf{M} \boldsymbol{\Phi}_{m} \hat{f}_{ \pm, 1}^{(0)}+\left(\hat{\lambda}_{ \pm}-\lambda_{1}\right) \mathbf{M} \boldsymbol{\Phi}_{m} \hat{f}_{ \pm, 1^{*}}^{(0)}=\hat{\mathbf{F}}_{ \pm}
$$

This equation differs from that obtained with a complex normal form style parametrisation since $\hat{\lambda}$ cannot be resonant with both $\lambda_{1}$ and $\bar{\lambda}_{1}$, but only with one of them. As explained in Section 3.3 . Eq. (79) must be complemented with proper orthogonality conditions. Since both graph style and real normal form style parametrisations imposes $\hat{\theta}_{ \pm, 1}^{(0)}=\hat{\theta}_{ \pm, 1^{*}}^{(0)}=0$ if $\hat{\lambda}_{ \pm}= \pm \omega_{m}$, from Eq. (58) the resulting relations are obtained:

$$
\begin{gathered}
\left(\hat{\lambda}_{ \pm}-\lambda_{1^{*}}\right) \boldsymbol{\Phi}_{m}^{\mathrm{T}} \mathbf{M} \hat{\boldsymbol{\Psi}}_{ \pm, \mathcal{I}}^{(0)}+\hat{f}_{ \pm, 1}^{(0)}+\hat{f}_{ \pm, 1^{*}}^{(0)}=0 \\
\left(\hat{\lambda}_{ \pm}-\lambda_{1}\right) \boldsymbol{\Phi}_{m}^{\mathrm{T}} \mathbf{M} \hat{\boldsymbol{\Psi}}_{ \pm, \mathcal{I}}^{(0)}+\hat{f}_{ \pm, 1}^{(0)}+\hat{f}_{ \pm, 1^{*}}^{(0)}=0 .
\end{gathered}
$$

By taking the difference between the two equations one has that $\boldsymbol{\Phi}_{m}^{\mathrm{T}} \mathbf{M} \hat{\mathbf{\Psi}}^{(0)}=0$. As a consequence:

$$
\hat{f}_{ \pm, 1}^{(0)}+\hat{f}_{ \pm, 1^{*}}^{(0)}=0
$$

Finally, using this last conditions and by projecting Eq. 79 onto the modal subspace, the following expressions are obtained:

$$
2 \mathfrak{i} \operatorname{Im}\left[\lambda_{1}\right] \hat{f}_{ \pm, 1}^{(0)}=\boldsymbol{\Phi}_{m}^{\mathrm{T}} \hat{\mathbf{F}}_{ \pm}, \quad 2 \mathrm{i} \operatorname{Im}\left[\lambda_{1^{*}}\right] \hat{f}_{ \pm, 1^{*}}^{(0)}=\boldsymbol{\Phi}_{m}^{\mathrm{T}} \hat{\mathbf{F}}_{ \pm}
$$

The resulting mass-orthogonality between zero-order mapping and master linear subspace is meaningful from a physical standpoint. In the case where the forcing has a spatial dependence that is purely co-linear with $\boldsymbol{\Phi}_{m}$, the resulting mapping $\hat{\mathbf{\Psi}}_{ \pm}^{(0)}$ vanishes. This explains for instance the excellent accuracy of the numerical results reported in past works that adopted such parametrisation styles to obtain ROMs from the autonomous problem only [22,32, 33, 35. A necessary remark is that in the special case of complex normal form styles, as for instance the one adopted in [36, the physical interpretation of the results is less obvious from a physical standpoint since also part of the forcing co-linear with $\boldsymbol{\Phi}_{m}$ is embedded within $\hat{\boldsymbol{\Psi}}_{ \pm}^{(0)}$. This implies that the computed whisker exhibits modulations in time along the plane associate to the master mode as the one depicted in Fig. 2 a.

The terms of the reduced dynamics for the non-autonomous part at order zero, as shown in Eq. (82, are directly proportional to the projection of the external force onto $\boldsymbol{\Phi}_{m}$. In mechanical systems, the most common case is to have $\boldsymbol{\Phi}_{m}^{\mathrm{T}} \hat{\mathbf{F}}_{ \pm} \neq 0$, which in turn yields zero-order resonant terms in the reduced dynamics. This condition is hereafter labelled primary resonance. Furthermore, higher order resonance conditions given by $\hat{\sigma}_{ \pm \mathcal{I}} \approx \lambda$ yield terms in the reduced dynamics that account for the change in configuration of the system during motion. This last result has special relevance for structures excited at large vibration amplitudes. Numerical examples illustrating the benefits of introducing high order terms in the non-autonomous developments are reported in Section 6.1.

A different resonance phenomenon is met in the peculiar case of a forcing orthogonal to the master linear subspace, i.e. such that $\boldsymbol{\Phi}_{m}^{\mathrm{T}} \hat{\mathbf{F}}_{ \pm}=0$. This condition is provided for instance by a forcing co-linear with a slave mode, hence zero-order terms of the non-autonomous reduced dynamics are zero as evidenced by Eq. 82 . If the slave mode is coupled to the master mode, then high order non-autonomous terms appear in the reduced dynamics. As underlined in previous Sections, for $\gamma=1$ only even order resonances are observed in the asymptotic development, consequently the terms introduced in the reduced dynamics by the non-autonomous part are at least $O\left(\left\|\mathbf{z}^{2}\right\|\right)$. The outcome is that the origin $\mathbf{z}$ of the system in presence of damping is an asymptotically stable fixed point and non trivial solutions are necessarily detached from it, i.e. isolated solutions may appear. This very specific case will be illustrated in Section 6.2. One can however note that practical conditions that yield isolated solutions in mechanical systems are rare when a single master mode is involved since in general the forcing is never perfectly orthogonal to the master mode and even a little amount of modal contribution to the master mode is sufficient to merge the isolated solutions with the main branch. 


\subsection{Secondary resonance with $\gamma=2$ and parametric excitation}

We now focus on the solution given by the reduction procedure when $\gamma=2$, i.e. when the excitation frequency is twice the natural frequency of the master mode. Since resonance effects appear already at order $\gamma-1$ for the non-autonomous part, the first consequences have to be analyzed at the first order of the development. Zero-order terms showing no resonance, the simplest choice thus consists in setting $\hat{f}_{ \pm, r \mathcal{I}}^{(0)}=0$, which in turn implies that the resulting non-autonomous mapping $\hat{\mathbf{\Psi}}_{ \pm}^{(0)}$ is provided by Eq. (72).

Since $\gamma=2$ the monomials associated to this case are of second order with a product of a term involving the forcing, and a term involving the normal coordinate z. More specifically, if $\Omega \approx 2 \omega_{m}$ then the associated trivial resonance conditions are:

$$
\begin{gathered}
\hat{\sigma}_{+, 1}=\hat{\lambda}_{+}+\lambda_{1^{*}}=+i \mathfrak{i} \Omega+\lambda_{1^{*}} \approx \lambda_{1}, \\
\hat{\sigma}_{-, 1}=\hat{\lambda}_{-}+\lambda_{1}=-i \mathfrak{\Omega}+\lambda_{1} \approx \lambda_{1^{*}} .
\end{gathered}
$$

In the case where a complex normal form is selected (recalling that this style of parametrisation give rise to the simplest possible formulation for the reduced dynamics), the following first-order non-autonomous reduced dynamics is obtained:

$$
\hat{\boldsymbol{f}}^{(1)}(\mathbf{z}, \Omega, t)=\left[\begin{array}{c}
\hat{f}_{+, 1\left\{1^{*}\right\}}^{(1)} \\
0
\end{array}\right] \mathrm{z}_{1^{*}} e^{\hat{\lambda}_{+} t}+\left[\begin{array}{c}
0 \\
\hat{f}_{-, 1^{*}\{1\}}^{(1)}
\end{array}\right] \mathrm{z}_{1} e^{\hat{\lambda}_{-} t}
$$

where the analytical expression of the $\hat{f}^{(1)}$ terms is provided in Appendix E and detailed treatment of their analytical expressions is provided in Section 6. As a main consequence, the reduced dynamics in this case makes appear terms that are the drivers for a parametric instability since being directly proportional to the forcing. Indeed, the product between the normal coordinates and the external forcing are typical of Mathieu-like equations, and induce parametric resonance when the excitation frequency is twice the eigenfrequency. This situation will be further analyzed in Section 6.3. In particular, the case of an axially loaded beam will be studied, and we will show that the ROM includes some terms in the non-autonomous part of the reduced dynamics, that are capable of reproducing the parametric instability.

To conclude this Section, let us remark that higher order resonance conditions are also met for $\gamma>2$. For increasing values of $\gamma$, the effects of such resonances appear from order $\gamma-1$ in the reduced dynamics of the non-autonomous part, meaning that their occurrence is somehow limited to larger amplitudes and more extreme excitation cases. As a general statement, this will give rise in the reduced dynamics to terms with product of the forcing associated to a higher order of the power of the normal coordinate $\mathrm{z}$, a situation that is prone to the emergence of isolated solutions to the system, detached from the fixed point $\mathbf{z}=\mathbf{0}$. A more complete analysis is left for future works.

\section{Realification}

In vibratory systems the eigenspectrum of the first-order formulation given in Eq. (17) is characterised by pairs of complex conjugate eigenvalues. As a consequence, adopting a diagonalised formulation for the linear part of the reduced dynamics enforces the complexification of the problem [28, hence making the resulting mappings and reduced dynamics complex-valued.

However, one can recover the real-valued nature of the original system. This operation, called realification, is thus the flip side of the complexification, and already attracted general comments and solutions, see e.g. 28. For vibratory systems, realification is based on the introduction of a linear transformation acting on the normal coordinates which preserves the mapped displacement $\mathbf{U}$ and velocity $\mathbf{V}$ fields. For instance one can set:

$$
\mathbf{a}=\mathbf{R z}
$$

with a the real-valued normal coordinates. The linear operator $\mathbf{R}$ is defined as in [35]:

$$
\mathbf{R}=\left[\begin{array}{cc}
-\mathfrak{i} \mathbf{I} & i \mathbf{I} \\
\mathbf{I} & \mathbf{I}
\end{array}\right]
$$

This is based on the simple observation that normal coordinates $\mathrm{z}_{j}$ and $\mathrm{z}_{j^{*}}$ are respectively complex conjugate and, as a result, real-valued mappings and reduced dynamics can be obtained by simply choosing 
as new coordinates real and imaginary parts of the complex normal coordinates:

$$
\begin{aligned}
\mathrm{a}_{j} & =\mathrm{z}_{j}+\mathrm{z}_{j+\mathrm{n}}=2 \operatorname{Re}\left[\mathrm{z}_{j}\right], \\
\mathrm{a}_{j+\mathrm{n}} & =\frac{\mathrm{z}_{j}-\mathrm{z}_{j+\mathrm{n}}}{\mathfrak{i}}=2 \operatorname{Im}\left[\mathrm{z}_{j}\right] .
\end{aligned}
$$

The realification procedure needs to the applied to both mappings and reduced dynamics resulting from the DPIM for all orders of expansion. This in turn provides the following relations between complex and real mappings:

$$
\begin{aligned}
& \sum_{\mathcal{I}} \stackrel{\circ}{\Psi}_{j \mathcal{I}}^{(p)} \pi_{\mathcal{I}}^{(p)}=\sum_{\mathcal{I}} \stackrel{\tilde{\Theta}}{\mathbf{\Psi}}_{j \mathcal{I}}^{(p)} \tilde{\pi}_{\mathcal{I}}^{(p)}, \\
& \sum_{\mathcal{I}} \stackrel{\circ}{\Upsilon}_{j \mathcal{I}}^{(p)} \pi_{\mathcal{I}}^{(p)}=\sum_{\mathcal{I}} \tilde{\check{\Upsilon}}_{j \mathcal{I}}^{(p)} \tilde{\pi}_{\mathcal{I}}^{(p)},
\end{aligned}
$$

where $(\tilde{\cdot})$ denotes realified quantities, and the $(\cdot)$ notation is used again to refer simultaneously to autonomous and non-autonomous quantities. Similar relations hold for the reduced dynamics with the same approach presented in [35]. The new monomials $\tilde{\pi}_{\mathcal{I}}^{(p)}$ have the same structure as the complex ones:

$$
\tilde{\pi}_{\mathcal{I}}^{(p)}=a_{i_{1}} a_{i_{2}} \ldots a_{i_{p}}, \quad \mathcal{I}=\left\{i_{1} i_{2} \ldots i_{p}\right\}
$$

Regarding the non-autonomous part, a further step is required to realify the system. Indeed, to uncouple harmonics during the solution to the homological equations, Euler relations are exploited to complexify the time dependence of the mapping. The inverse relation is then used to fully realify non-autonomous mappings and reduced dynamics via $\cos (\Omega t)=\left(e^{+\mathfrak{i} \Omega t}+e^{-\mathfrak{i} \Omega t}\right) / 2$ and $\sin (\Omega t)=\left(e^{+\mathfrak{i} \Omega t}-e^{-\mathfrak{i} \Omega t}\right) / 2 \mathfrak{i}$. An important remark regarding this last step is that, since the applied forcing is real-valued, i.e. $\overline{\hat{\mathbf{F}}}=\hat{\mathbf{F}}$, the following conditions hold:

$$
\overline{\hat{\mathbf{F}}}_{+}=\hat{\mathbf{F}}_{-}, \quad \overline{\hat{\mathbf{F}}}_{-}=\hat{\mathbf{F}}_{+},
$$

As a consequence we have that:

$$
\hat{\mathbf{\Psi}}_{j, \mathcal{I}}^{(p)}=\operatorname{conj}\left[\hat{\mathbf{\Psi}}_{j^{*}, \mathcal{I}^{*}}^{(p)}\right], \quad \hat{\boldsymbol{\Upsilon}}_{j, \mathcal{I}}^{(p)}=\operatorname{conj}\left[\hat{\boldsymbol{\Upsilon}}_{j^{*}, \mathcal{I}^{*}}^{(p)}\right], \quad \hat{f}_{j, s \mathcal{I}}^{(p)}=\operatorname{conj}\left[\hat{f}_{j^{*}, s^{*} \mathcal{I}^{*}}^{(p)}\right] .
$$

This last result is remarkably important from a computational standpoint. Indeed, for each excitation frequency, there is an associated pair of complex conjugate eigenvalues $+i \mathfrak{l}$ and $-\mathfrak{i} \Omega$. However, from Eq. (91) it is possible to perform the complete parametrisation procedure for a single forcing eigenvalue, and then exploit the symmetry relations to derive mappings and reduced dynamics of the complex conjugate eigenvalue. This is in turn a natural consequence of the redundancy of one of the auxiliary variables $\hat{a}$ introduced in Eq. (24), since only one of them can be used by introducing the phase $\Theta=\omega t$ instead of the trigonometric functions.

The resulting real-valued mappings and reduced dynamics are finally expressed as:

$$
\begin{aligned}
\tilde{\hat{\boldsymbol{\Psi}}}(\mathbf{a}, \boldsymbol{\Omega}, t) & =\sum_{\Omega_{i} \in \boldsymbol{\Omega}} \tilde{\hat{\boldsymbol{\Psi}}}_{\Omega_{i}, c}(\mathbf{a}) \cos \left(\Omega_{i} t\right)+\tilde{\hat{\boldsymbol{\Psi}}}_{\Omega_{i}, s}(\mathbf{a}) \sin \left(\Omega_{i} t\right), \\
\tilde{\hat{\boldsymbol{\Upsilon}}}(\mathbf{a}, \boldsymbol{\Omega}, t) & =\sum_{\Omega_{i} \in \boldsymbol{\Omega}} \tilde{\hat{\boldsymbol{\Upsilon}}}_{\Omega_{i}, c}(\mathbf{a}) \cos \left(\Omega_{i} t\right)+\tilde{\hat{\boldsymbol{\Upsilon}}}_{\Omega_{i}, s}(\mathbf{a}) \sin \left(\Omega_{i} t\right), \\
\tilde{\hat{\boldsymbol{f}}}(\mathbf{a}, \boldsymbol{\Omega}, t) & =\sum_{\Omega_{i} \in \boldsymbol{\Omega}} \tilde{\hat{\boldsymbol{f}}}_{\Omega_{i}, c}(\mathbf{a}) \cos \left(\Omega_{i} t\right)+\tilde{\hat{\boldsymbol{f}}}_{\Omega_{i}, s}(\mathbf{a}) \sin \left(\Omega_{i} t\right) .
\end{aligned}
$$

A last remark concerns the choice of the transformation exploited to realify the system. Indeed, this choice is not unique. For instance in 33 the following transformation was applied:

$$
\mathbf{R}=\left[\begin{array}{cc}
-i \boldsymbol{\Omega}^{*} & \mathbf{i} \boldsymbol{\Omega}^{*} \\
\mathbf{I} & \mathbf{I}
\end{array}\right]
$$

with $\Omega^{*}$ a diagonal matrix that stores the master modes eigenfrequencies. This last transformation has the benefit of yielding two sets of real-valued normal coordinates $\mathbf{r}$ and $\mathbf{s}$, which can be directly related to the real normal variables used in [13. Alternatively, a polar representation as the one adopted by Haller et al. 29, 34 can be adopted:

$$
\begin{aligned}
\mathrm{a}_{j} & =\rho_{j} \cos \alpha_{j}, \\
\mathrm{a}_{j+\mathrm{n}} & =\rho_{j} \sin \alpha_{j},
\end{aligned}
$$


with $\rho_{j}$ amplitude, and $\alpha_{j}$ phase. This last transformation is particularly helpful when deriving analytic expressions for backbones, isolas, and frequency response curves in the case of single master mode reduction. However, as evidenced in [36], it yields a reduced dynamics that diverges in the limit of vanishing amplitudes when $\rho_{j} \rightarrow 0$, hence making it of little use when multiple master modes are involved e.g., in presence of internal resonances.

\section{Numerical results}

In this Section, several examples are detailed, covering applications of both academic and industrial interest. We follow the order of the discussion proposed in Section 4 and illustrate the different scenarios with the appearance of resonances for increasing values of $\gamma$. The industrial example is a MEMS resonator with complex geometry. All the analyses are validated with full-order simulations realised with an harmonic balance technique directly implemented on the finite element model, and denoted in the remainder as HBFEM solutions 62. Numerical integration of the ROMs is performed using the continuation packages MatCont [63], ManLab [64], and BifurcationKit [65].

We recall that, according to the conventions set in Eq. $(22)$ and Section 4 , the normal variables vector has two entries that are indexed with 1 and $2=1^{*}$ and that $\lambda_{1}=\Lambda_{m}, \lambda_{1^{*}}=\Lambda_{m}, \boldsymbol{\phi}_{1}=\boldsymbol{\phi}_{1^{*}}=\boldsymbol{\Phi}_{m}$. Moreover, in all the examples, the ROM is computed for a single excitation frequency $\Omega$, which is selected as the eigenfrequency of the master mode $\omega_{m}$ for all cases, except for the parametric resonance in Section 6.3 . where it has been set at $\Omega=2 \omega_{m}$.

Finally, instead of using the abstract label $m$ to denote the master mode, different indices will be used in most cases, in order to better stress the physical interpretation of the mode.

6.1 Large amplitude vibrations of a forced cantilever beam

a)

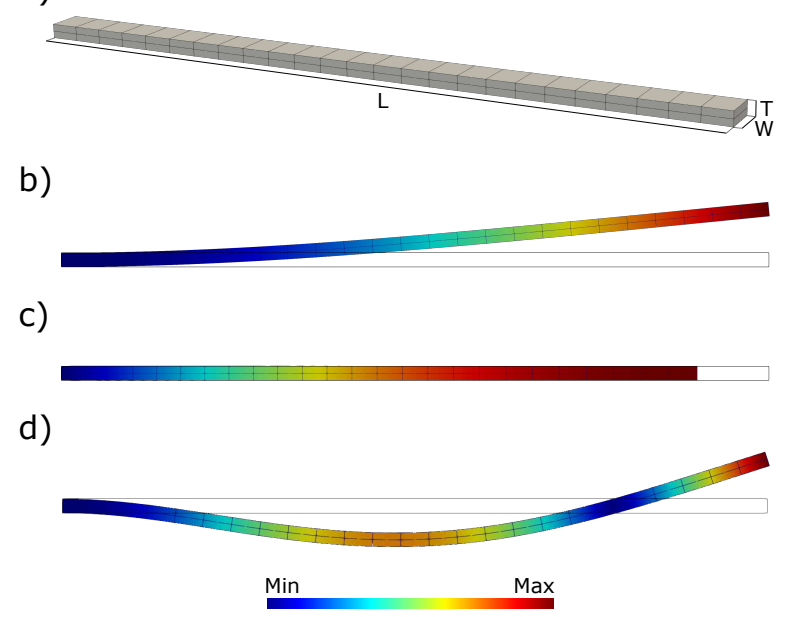

Fig. 3 (a) Cantilever geometry. The structure has a length $\mathrm{L}$ equal to $1 \mathrm{~m}$, a width $\mathrm{W}$ equal to $0.05 \mathrm{~m}$, and a thickness $\mathrm{T}$ equal to $0.02 \mathrm{~m}$. Pictures (b), (c), and (d) depict the displacement fields associated to the first bending mode $\boldsymbol{\Phi}_{B_{1}}$, the first axial mode $\boldsymbol{\Phi}_{A_{1}}$ (which is mode number 10 in the order of increasing frequencies for this specific beam), and the second bending mode $\boldsymbol{\Phi}_{B_{2}}$, respectively. Displacement magnitude is scaled to improve the readability of the images.

The first case under study is a cantilever beam, shown in Fig. 3(a), of length $1 \mathrm{~m}$, width $0.05 \mathrm{~m}$, and thickness $0.02 \mathrm{~m}$. Homogeneous Dirichlet conditions on the displacement are imposed on the left end of the beam. Adopted material properties are those of titanium, i.e. isotropic behaviour with Young's modulus 104 GPa, Poisson's ratio 0.3, and density $4400 \mathrm{~kg} / \mathrm{m}^{3}$. The structure is excited with an harmonic body force proportional to the first bending mode. The model associated to the structure under consideration is therefore formulated as:

$$
\int_{\mathrm{B}} \rho \ddot{\boldsymbol{u}} \cdot \boldsymbol{w} d \mathrm{~B}+\int_{\mathrm{B}} \mathbf{P}: \nabla^{T} \boldsymbol{w} d \mathrm{~B}=\kappa\left[\int_{\mathrm{B}} \rho \boldsymbol{\Phi}_{B_{1}} \cdot \boldsymbol{w} d \mathrm{~B}\right] \cos (\Omega t), \quad \forall \boldsymbol{w} \in \mathcal{C}(\mathbf{0}),
$$

where $\boldsymbol{\Phi}_{B_{1}}$ stands for the first bending mode, $\kappa$ is a load multiplier, and $\Omega$ the forcing frequency. In this Section, bending modes will be denoted with the subscript $B$ and a subscript corresponding to their order 
with respect to increasing frequencies, while axial modes will be denoted with the subscript $A$. Fig. 3(b-d) respectively shows the mode shapes corresponding to $\boldsymbol{\Phi}_{B_{1}}, \boldsymbol{\Phi}_{A_{1}}$ and $\boldsymbol{\Phi}_{B_{2}}$. The geometry is discretised using 27-nodes quadratic hexahedral elements for a total of 2295 degrees of freedom and the following discrete system is obtained, where the cosine is decomposed in its imaginary exponential parts:

$$
\mathbf{M} \ddot{\mathbf{U}}+\mathbf{C} \dot{\mathbf{U}}+\mathbf{K U}+\boldsymbol{G}(\mathbf{U}, \mathbf{U})+\boldsymbol{H}(\mathbf{U}, \mathbf{U}, \mathbf{U})=\frac{1}{2} \kappa \mathbf{M} \boldsymbol{\Phi}_{B_{1}}\left(e^{+i \Omega t}+e^{-i \Omega t}\right) .
$$

A mass-proportional damping with $\mathbf{C}=\left(\omega_{B_{1}} / 1000\right) \mathbf{M}$ has been added. The forcing frequency being selected in the vicinity of the fundamental eigenfrequency, $\Omega \simeq \omega_{B_{1}}$, hence this case illustrates the theoretical results reported in Section 4.2 , with $\gamma=1$.

The analysis shown in this Section complements the one reported in [35] by taking into account forcing and damping, and thus enabling the computation of frequency-response curve (FRC). In the undamped scenario, an important quadratic coupling between $\boldsymbol{\Phi}_{B_{1}}$ and $\boldsymbol{\Phi}_{A_{1}}$ induces a folding of the manifold associated with the first bending mode. A consequence, shown in [35], is that only a normal form style parametrisation is able to retrieve the backbone of the first mode up to very large amplitudes. Since no internal resonance exists between the fundamental frequencies, we will formulate a ROM with a single master mode, i.e. the fundamental bending mode which is directly excited by the load.

In order to obtain a versatile ROM that can be used for parametric variations of the forcing (amplitude and excitation frequency to compute the FRC), the reduction is performed for a single excitation frequency, as discussed in Section 3.1. In this case, the selected forcing frequency used to compute the ROM is $\Omega=\omega_{B_{1}}$. Numerical simulations are repeated for $\kappa$ values equal to $1,2,3,4$, and $5 \mathrm{~m} / \mathrm{s}^{2}$, in order to monitor the evolution of the computed amplitude response for increasing amplitudes. An order 7 Fourier expansion is used in the HBFEM.

a)

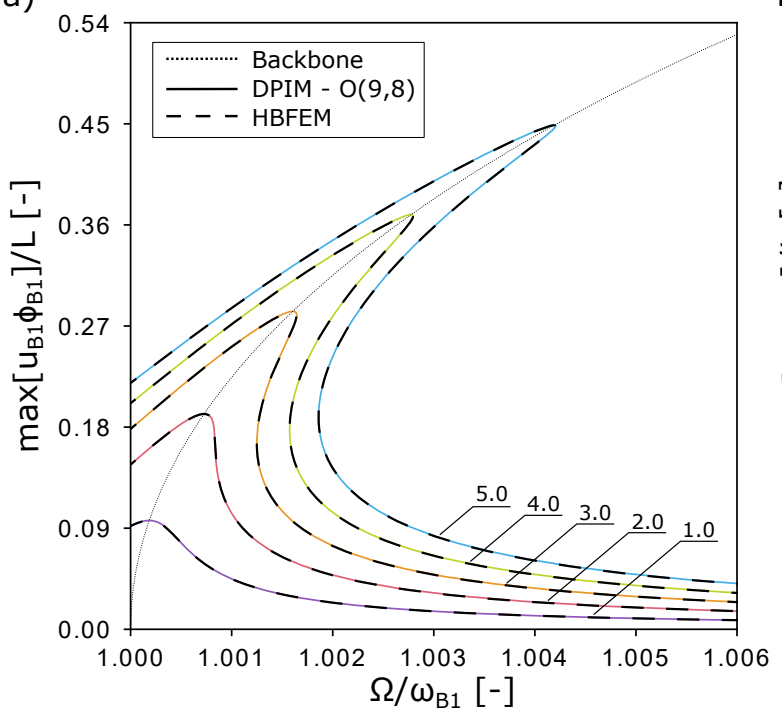

b)

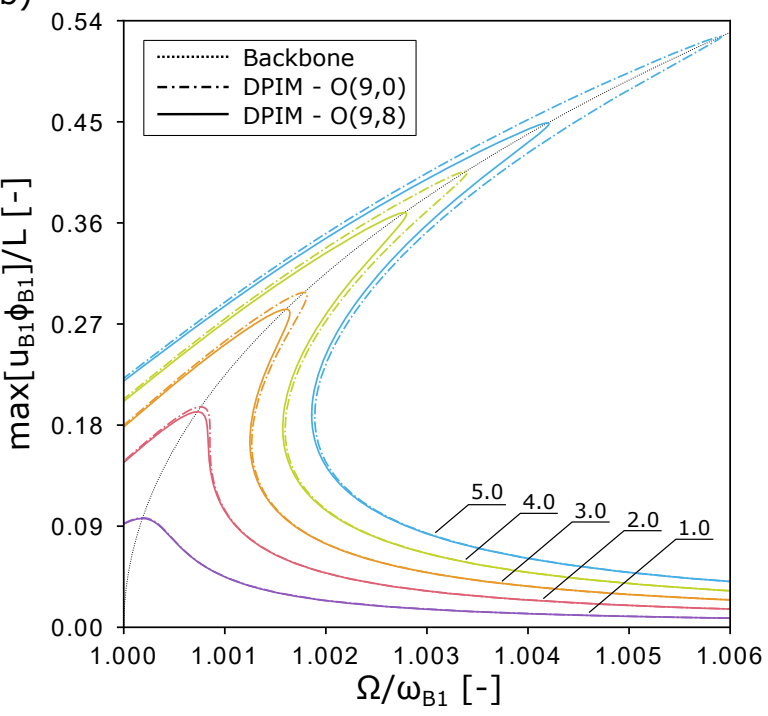

Fig. 4 Cantilever beam. (a) Comparison between full-order HBFEM simulations and the reduced model obtained with the DPIM method with order 9 parametrisation of the autonomous problem and an order 8 parametrisation of the nonautonomous part. (b) Comparison between order 8 and order 0 parametrisation of the non-autonomous part, keeping the parametrisation order of the autonomous part equal to 9 . Tags denote the values of the load multiplier $\kappa\left[\mathrm{m} / \mathrm{s}^{2}\right]$. In increasing order, the dimensionless loading multiplier $\check{\kappa}$ as defined in Eq. 103 is equal to $9.72 \cdot 10^{-5}, 1.94 \cdot 10^{-4}, 2.92 \cdot 10^{-4}$, $3.88 \cdot 10^{-4}$, and $4.86 \cdot 10^{-4}$.

A complex normal form style is used for all the calculations. The autonomous mappings and reduced dynamics are first expanded up to order 9, thus guaranteeing convergence, as discussed next. On the contrary, the parametrisation order of the non-autonomous part is taken either equal to zero or 8 . Selecting a zero-order for the time-dependent part leads to the same results previously obtained with the direct normal form (DNF) 32,33, but also with the cases shown in [34] where a complex-valued normal form is used. The aim of this first example is to underline the importance of the higher-order terms of the non-autonomous part to retrieve a perfect accuracy on the FRC with large amplitudes.

The results are collected in Fig. 4. where the modal amplitude $\mathrm{u}_{B_{1}}$ of the first mode, normalized by the length of the beam, is reported. Fig. 4(a) compares the results of the ROM with orders $\mathcal{O}(9,8)($ using a 
notation that will be used in the remainder of the paper, the first integer refers to the autonomous development and the second to the non-autonomous part) to the full-order solutions, showing a perfect agreement up to very large oscillation amplitudes reaching almost half the length of the beam. This result underlines that efficient parametric ROMs can be obtained using a single excitation frequency in the parametrization procedure. Furthermore, computational performance are excellent. Indeed, while HBFEM simulations required a total of 8 hours to retrieve the nonlinear dynamic response of the structure, the reduced model required less than 15 minutes in total.

Fig. 4(b), by comparing orders $\mathcal{O}(9,0)$ and $\mathcal{O}(9,8)$, shows the importance of taking into account the nonautonomous part in order to obtain a correct result. The information added by the non-autonomous part is of crucial importance to recover the correct amplitude and in particular the maximum of the FRC which is close to the saddle-node bifurcation point. As awaited, the discrepancy increases with the oscillation amplitude, and the $\mathcal{O}(9,0)$ solution importantly overestimates the peak of the FRC. This also complements the analysis already shown in [22], where the effect of the damping on such FRC was documented. Here, the results clearly underline that not only an incorrect approximation of the damping might explain an incorrect estimation at resonance, but also how the forcing is treated.

Since the difference between high and low-order parametrisations involves both the location in amplitude and frequency of the maximum point of the FRC, let us investigate more closely how the forcing affects the reduced dynamics. Indeed, while the predicted amplitudes are strongly connected to the mappings $\boldsymbol{\Psi}(\mathbf{z})$ and $\hat{\boldsymbol{\Psi}}(\mathbf{z})$, the frequency of the saddle-node points is only controlled by the reduced dynamics. The eigenvalues of the master mode write $\lambda_{1}=\Lambda_{B_{1}}=-\xi_{B_{1}} \omega_{B_{1}}+i \omega_{B_{1}} \sqrt{1-\xi_{B_{1}}^{2}}$ and $\lambda_{1^{*}}=\bar{\Lambda}_{B_{1}}=-\xi_{B_{1}} \omega_{B_{1}}-\mathfrak{i} \omega_{B_{1}} \sqrt{1-\xi_{B_{1}}^{2}}$, while those of the forcing are $\hat{\lambda}_{ \pm}= \pm i \omega_{B_{1}}$. Since we are in the case $\gamma=1$, see Section 4.2 , resonances are possible only for even parametrisation orders. By restricting the derivation here to $\varepsilon^{1}$ homological equations, we have the following trivial resonance conditions at order zero:

$$
\begin{aligned}
& \hat{\lambda}_{+} \approx \lambda_{1}, \\
& \hat{\lambda}_{-} \approx \lambda_{1 *} .
\end{aligned}
$$

At order one, no trivial resonances are observed, hence $\hat{\mathbf{f}}^{(1)}=\mathbf{0}$. At order two instead, new trivial resonance conditions are satisfied, as:

$$
\begin{aligned}
\hat{\lambda}_{+}+\lambda_{1}+\lambda_{1^{*}} & \approx \lambda_{1}, \\
\hat{\lambda}_{+}+\lambda_{1^{*}}+\lambda_{1^{*}} & \approx \lambda_{1^{*}}, \\
\hat{\lambda}_{-}+\lambda_{1}+\lambda_{1^{*}} & \approx \lambda_{1^{*}}, \\
\hat{\lambda}_{-}+\lambda_{1}+\lambda_{1} & \approx \lambda_{1} .
\end{aligned}
$$

The presence of these trivial resonance relationships, here detailed for the non-autonomous part, leads to a reduced dynamics which, by taking into account the first two meaningful orders for the terms introduced by the forcing, reads:

$$
\begin{aligned}
& {\left[\begin{array}{l}
\dot{\mathrm{z}}_{1} \\
\dot{\mathrm{z}}_{1^{*}}
\end{array}\right]=\boldsymbol{f}(\mathbf{z})+\kappa\left(\left[\begin{array}{l}
\hat{f}_{+, 1}^{(0)} \\
0
\end{array}\right] e^{+i \Omega t}+\left[\begin{array}{l}
0 \\
\hat{f}_{-, 1^{*}}^{(0)}
\end{array}\right] e^{-i \Omega t}\right)} \\
& +\kappa\left(\left[\begin{array}{l}
\hat{f}_{-, 1\{11\}}^{(2)} \\
0
\end{array}\right] e^{-i \Omega t}\right) \mathrm{z}_{1}^{2}+\kappa\left(\left[\begin{array}{l}
0 \\
\hat{f}_{+, 1^{*}\left\{1^{*} 1^{*}\right\}}^{(2)}
\end{array}\right] e^{+i \Omega t}\right) \mathrm{z}_{1^{*}}^{2} \\
& +\kappa\left(\left[\begin{array}{l}
\hat{f}_{+, 1\left\{11^{*}\right\}}^{(2)}+\hat{f}_{+, 1\left\{1^{*} 1\right\}}^{(2)} \\
0
\end{array}\right] e^{+i \Omega t}+\left[\begin{array}{l}
0 \\
\hat{f}_{-, 1^{*}\left\{11^{*}\right\}}^{(2)}+\hat{f}_{-, 1^{*}\left\{1^{*} 1\right\}}^{(2)}
\end{array}\right] e^{-i \Omega t}\right) \mathrm{z}_{1} \mathrm{z}_{1 *}+O\left(\left\|\mathbf{z}^{3}\right\|\right),
\end{aligned}
$$

hence highlighting the appearance of higher-order terms in $\mathbf{z}$.

In order to get a complete picture of the terms that arise from the non-autonomous part, it is worth inspecting the coefficients $\hat{\mathbf{f}}^{(0)}$ and $\hat{\mathbf{f}}^{(2)}$. From Appendix E one obtains:

$$
\begin{aligned}
\hat{f}_{+, 1}^{(0)} & =\frac{\boldsymbol{\Phi}_{B_{1}}^{\mathrm{T}}}{\lambda_{1}-\lambda_{1^{*}}} \hat{\mathbf{F}}_{+}, \\
\hat{f}_{-, 1^{*}}^{(0)} & =\frac{\boldsymbol{\Phi}_{B_{1}}^{\mathrm{T}}}{\lambda_{1^{*}}-\lambda_{1}} \hat{\mathbf{F}}_{-},
\end{aligned}
$$


where $\lambda_{1}-\lambda_{1^{*}}=2 \operatorname{Im}\left[\lambda_{1}\right]=2 i \omega_{B_{1}} \sqrt{1-\xi_{B_{1}}^{2}}$ and $\lambda_{1^{*}}-\lambda_{1}=2 \operatorname{Im}\left[\lambda_{1^{*}}\right]=-2 i \omega_{B_{1}} \sqrt{1-\xi_{B_{1}}^{2}}$. Expressions of $\hat{f}^{(2)}$ coefficients are much longer and are reported in Appendix E. They all share the same shape so that only one of them is reported here for the sake of brevity:

$$
\begin{aligned}
\hat{f}_{-, 1\{11\}}^{(2)}= & \frac{\boldsymbol{\Phi}_{B_{1}}^{\mathrm{T}}}{\lambda_{1}-\lambda_{1^{*}}}\left[-\mathbf{M}\left[\mathbf{\Upsilon}_{\left\{1^{*} 11\right\}}^{(3)} \hat{f}_{-, 1^{*}}^{(0)}+\mathbf{\Upsilon}_{\left\{11^{*} 1\right\}}^{(3)} \hat{f}_{-, 1^{*}}^{(0)}+\mathbf{\Upsilon}_{\left\{111^{*}\right\}}^{(3)} \hat{f}_{-, 1^{*}}^{(0)}\right]\right. \\
& \left.+2 \boldsymbol{G}\left(\boldsymbol{\Psi}_{\{11\}}^{(2)}, \hat{\boldsymbol{\Psi}}_{-}^{(0)}\right)+2 \boldsymbol{G}\left(\boldsymbol{\Phi}_{B_{1}}, \hat{\boldsymbol{\Psi}}_{-,\{1\}}^{(1)}\right)+3 \boldsymbol{H}\left(\boldsymbol{\Phi}_{B_{1}}, \boldsymbol{\Phi}_{B_{1}}, \hat{\mathbf{\Psi}}_{-}^{(0)}\right)\right] \\
+ & \frac{\lambda_{1^{*}} \boldsymbol{\Phi}_{B_{1}}^{\mathrm{T}}}{\lambda_{1}-\lambda_{1^{*}}}\left[-\mathbf{M}\left[\boldsymbol{\Psi}_{\left\{1^{*} 11\right\}}^{(3)} \hat{f}_{-, 1^{*}}^{(0)}+\boldsymbol{\Psi}_{\left\{11^{*} 1\right\}}^{(3)} \hat{f}_{-, 1^{*}}^{(0)}+\boldsymbol{\Psi}_{\left\{111^{*}\right\}}^{(3)} \hat{f}_{-, 1^{*}}^{(0)}\right]\right],
\end{aligned}
$$

One can note in particular that $\hat{\mathbf{f}}^{(0)}$ coefficients are directly proportional to the forcing. For $\hat{\mathbf{f}}^{(2)}$ coefficients, the forcing terms also appears through the presence of $\hat{\mathbf{f}}^{(0)}$ in their expressions, together with quadratic and cubic terms $\mathbf{G}$ and $\mathbf{H}$, mixing the lower order mappings where the forcing is also present. For the cantilever beam, both quadratic and cubic terms play an important role and convey the lower order terms through the products. For instance, since $\hat{\boldsymbol{\Psi}}^{(0)}$ is directly proportional to $\boldsymbol{\Phi}_{B_{1}}$ (see Section 4.2 ), one has that $\boldsymbol{\Phi}_{B_{1}}^{\mathrm{T}} \boldsymbol{G}\left(\boldsymbol{\Psi}_{\{11\}}^{(2)}, \hat{\Psi}_{-}^{(0)}\right)$ is proportional to $\boldsymbol{\Phi}_{B_{1}}^{\mathrm{T}} \boldsymbol{G}\left(\boldsymbol{\Psi}_{\{11\}}^{(2)}, \boldsymbol{\Phi}_{B_{1}}\right)$. Moreover, $\boldsymbol{\Psi}_{\{11\}}^{(2)}$ embeds the contribution of the axial mode $\boldsymbol{\Phi}_{A_{1}}$, so that $\boldsymbol{\Phi}_{B_{1}}^{\mathrm{T}} \boldsymbol{G}\left(\boldsymbol{\Psi}_{\{11\}}^{(2)}, \hat{\boldsymbol{\Psi}}_{-}^{(0)}\right)$ is finally directly proportional to $\boldsymbol{\Phi}_{B_{1}}^{\mathrm{T}} \boldsymbol{G}\left(\boldsymbol{\Phi}_{A_{1}}, \boldsymbol{\Phi}_{B_{1}}\right)$. Interestingly, this last coefficient corresponds to the quadratic modal coupling coefficient appearing naturally in the equations of motion of flat symmetric structures and it is known to have a very large value, see e.g. 8]. Similarly, we have that:

$$
\boldsymbol{\Phi}_{B_{1}}^{\mathrm{T}} \boldsymbol{H}\left(\boldsymbol{\Phi}_{B_{1}}, \boldsymbol{\Phi}_{B_{1}}, \hat{\Psi}_{-}^{(0)}\right) \propto \boldsymbol{\Phi}_{B_{1}}^{\mathrm{T}} \boldsymbol{H}\left(\boldsymbol{\Phi}_{B_{1}}, \boldsymbol{\Phi}_{B_{1}}, \boldsymbol{\Phi}_{B_{1}}\right) .
$$

This last term corresponds to the cubic modal coefficient of the first bending mode, which is simply derived when using a Galerkin projection onto the linear modes. This term is also very large for such structure, hence explaining the large effect provided by second-order non-autonomous coefficients on the expected FRC of the system. This is reflected for instance in Fig. 4(b), where the amplitude of the response is severely influenced by these new terms.

a)

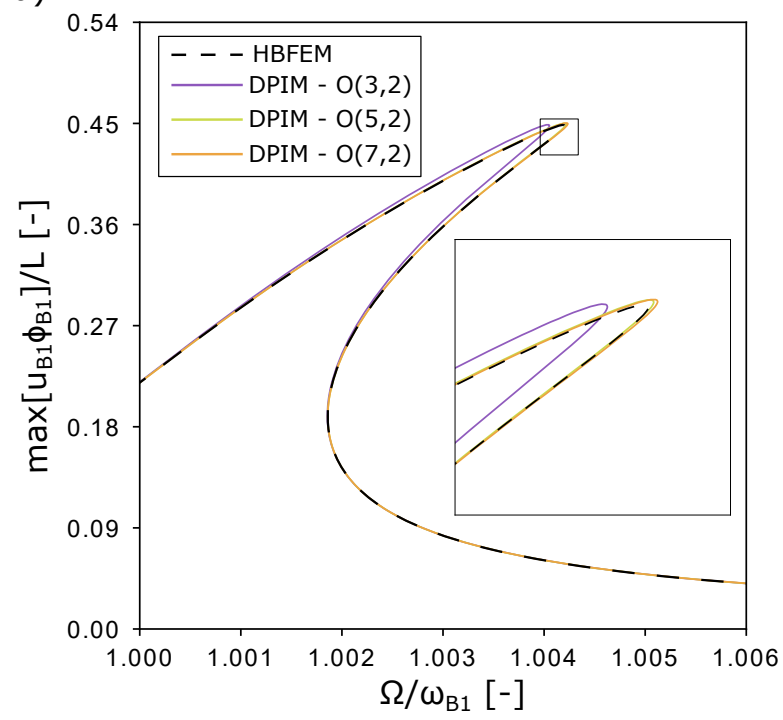

b)

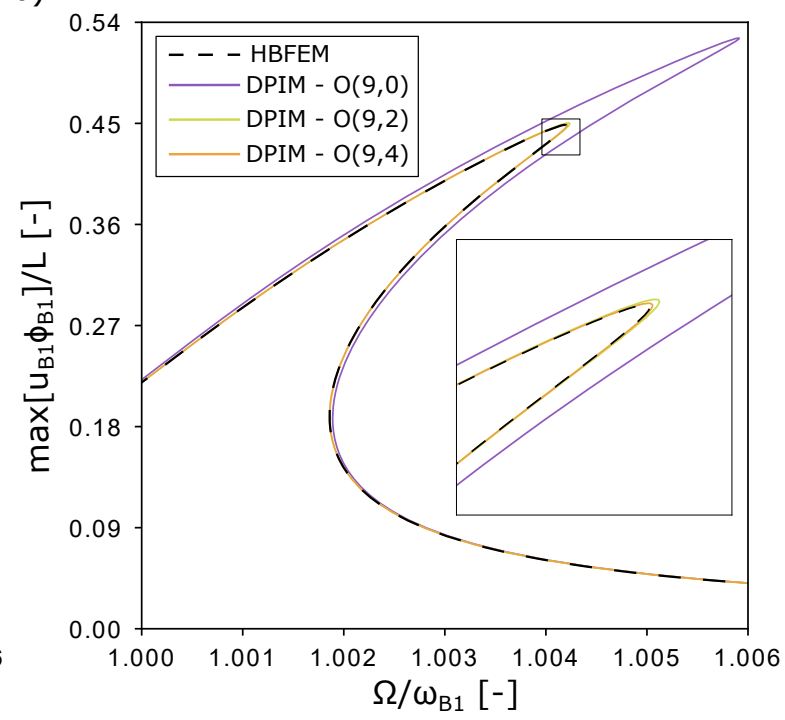

Fig. 5 Cantilever beam. DPIM convergence by: (a) changing the expansion order of the autonomous part, and (b) changing the order of the non-autonomous part.

The convergence of the ROM with respect to increasing the order of the expansions is now illustrated. Fig. 5(a) first reports the convergence of the autonomous part, by increasing it from 3 to 7 , while keeping the time-dependent terms at order 2 . We consider only odd values for the $\varepsilon^{0}$ terms, since the trivial resonances appear only at odd orders. On the other hand, the new trivial resonances due to the forcing appear at even orders and thus only even orders are considered in the convergence study of the non-autonomous part. The load multiplier $\kappa$ is here selected as $\kappa=5 \mathrm{~m} / \mathrm{s}^{2}$ in order to study the convergence for the maximum 
vibration amplitude tested, corresponding to almost half the length of the beam. Fig. 5(a) clearly shows that increasing the order of the autonomous part allows retrieving the correct type of nonlinearity, in line with the backbone curve computed and shown in [35. The convergence is rather fast, as minimal differences are observed above order 5 .

Let us now comment the convergence of the non-autonomous part, by setting the autonomous expansion to order 9 , where convergence is guaranteed. Results for order 0 of the time-dependent terms have already been shown in Fig. 4(b), underlining that this was clearly insufficient. Fig. 5(b) shows that the convergence is also fast for this part, the order 2 already showing a good accuracy, while perfect match with the fullorder solution is obtained starting with an order 4 . Indeed, the FRC with order 6 , not reported in the figure for the sake of clarity, is perfectly superimposed to order 4 . All in all, order $\mathcal{O}(7,4)$ already gives excellent results and the expansion used in the previous analysis, i.e. $\mathcal{O}(9,8)$, clearly referred to a converged result.

At last, let us introduce a new quantity $\check{\kappa}$ that will allow quantifying the validity of the small forcing assumption throughout this Section. The forcing parameter $\kappa$ can be normalised as:

$$
\check{\kappa}=\frac{\operatorname{Max}\left[\boldsymbol{\Phi}_{m}\right]}{\omega_{m}^{2} \mathrm{~s}} \kappa,
$$

with $\operatorname{Max}\left[\boldsymbol{\Phi}_{m}\right]$ the maximum amplitude value of the master mode shape, $\omega_{m}$ the angular frequency of the master mode, and s a characteristic displacement of the structure. Since this characteristic displacement is introduced to quantify the amount of forcing needed to excite the geometric nonlinearity, in this cantilever example it has been selected as the length $L$. For the beam under study, $\operatorname{Max}\left[\boldsymbol{\Phi}_{B_{1}}\right]=0.954$, and the eigenfrequency of the first mode is $99.08 \mathrm{rad} / \mathrm{s}$. Hence $\check{\kappa}=4.86 \cdot 10^{-4} \ll 1$, which is consistent with the assumption of small forcing.

Overall, this Section has been devoted to the analysis of the forced-damped response of a cantilever up to very large amplitudes, with a ROM containing a single master coordinate, illustrating the primary resonance scenario highlighted in Section 4.2 with $\gamma=1$. The results underline that a zero-order parametrisation of the non-autonomous part of the forced system is not sufficient to get a perfectly converged solution for the FRC at this range of amplitudes, and a second-order development is required. However, once this main effect captured, the ROM shows then fast convergence to the exact, full-order solution. We now turn to the particular case analysed in Section 4.2 of a main resonance without excitation term on the master mode, a case prone to see the birth of isolated solutions.

\subsection{Isolated solutions bifurcating from the main branch}

In this Section we consider the specific loading scenario where the excitation force is spatially orthogonal to the eigenmode that has the same frequency as the forcing. As detailed in Section 4.2 zero and first order non-autonomous terms of the reduced dynamics vanish in such loading scenario, and as a result only isolated solutions might appear.

The system under investigation is the same cantilever beam introduced in Section 6.1, the only difference being the excitation force which is now assumed to have the shape of the second bending mode $\boldsymbol{\Phi}_{B_{2}}$, whose displacement field is shown in Fig. 3(d). The model thus reads as:

$$
\int_{\mathrm{B}} \rho \ddot{\boldsymbol{u}} \cdot \boldsymbol{w} d \mathrm{~B}+\int_{\mathrm{B}} \mathbf{P}: \nabla^{T} \boldsymbol{w} d \mathrm{~B}=\kappa\left[\int_{\mathrm{B}} \rho \boldsymbol{\Phi}_{B_{2}} \cdot \boldsymbol{w} d \mathrm{~B}\right] \cos (\Omega t), \quad \forall \boldsymbol{w} \in \mathcal{C}(\mathbf{0}) .
$$

The system is still driven in the vicinity of the eigenfrequency of the fundamental mode: $\Omega \approx \omega_{B_{1}}$. The resulting system of differential equations obtained by discretising the system with finite elements is:

$$
\mathbf{M} \ddot{\mathbf{U}}+\mathbf{C U}+\mathbf{K U}+\boldsymbol{G}(\mathbf{U}, \mathbf{U})+\boldsymbol{H}(\mathbf{U}, \mathbf{U}, \mathbf{U})=\frac{1}{2} \kappa \mathbf{M} \boldsymbol{\Phi}_{B_{2}}\left(e^{+i \Omega t}+e^{-i \Omega t}\right) .
$$

where the damping matrix has been selected in this example as $\mathbf{C}=\alpha \mathbf{M}+\beta \mathbf{K}$ with $\alpha=5 \cdot 10^{-4} s^{-1}$ and $\beta=10^{-9} s$. The system is simulated for five values of the load multiplier $\kappa: 250,275,300,325$, and $350 \mathrm{~m} / \mathrm{s}^{2}$.

The reduced dynamics is derived by selecting the fundamental bending mode as master, and the complex normal form style is used. The ROM is computed for a single value of the forcing frequency, selected here as $\Omega=\omega_{B_{1}}$. The first terms of the reduced dynamics can be made explicit as:

$$
\begin{aligned}
{\left[\begin{array}{l}
\dot{\mathbf{z}}_{1} \\
\dot{\mathbf{z}}_{1 *}
\end{array}\right]=\boldsymbol{f}(\mathbf{z})+} & \kappa\left(\left[\begin{array}{l}
\hat{f}_{-, 1\{11\}}^{(2)} \\
0
\end{array}\right] e^{-i \Omega t}\right) \mathrm{z}_{1}^{2}+\kappa\left(\left[\begin{array}{l}
0 \\
\hat{f}_{+, 1^{*}\left\{1^{*} 1^{*}\right\}}^{(2)}
\end{array}\right] e^{+i \Omega t}\right) \mathrm{z}_{1^{*}}^{2} \\
& +\kappa\left(\left[\begin{array}{l}
\hat{f}_{+, 1\left\{11^{*}\right\}}^{(2)}+\hat{f}_{+, 1\left\{1^{*} 1\right\}}^{(2)} \\
0
\end{array}\right] e^{+i \Omega t}+\left[\begin{array}{l}
0 \\
\hat{f}_{-, 1^{*}\left\{11^{*}\right\}}^{(2)}+\hat{f}_{-, 1^{*}\left\{1^{*} 1\right\}}^{(2)}
\end{array}\right] e^{-i \Omega t}\right) \mathrm{z}_{1} \mathrm{z}_{1^{*}}+O\left(\left\|\mathbf{z}^{3}\right\|\right)
\end{aligned}
$$


where the expansion has been limited to the first non-autonomous terms for the present discussion. As announced, zero-order terms are not present since the projection of the forcing onto the master mode $\boldsymbol{\Phi}_{B_{1}}$ is zero. Since non-autonomous terms are at least $O\left(\left\|\mathbf{z}^{2}\right\|\right)$, the origin $\mathbf{z}=\mathbf{0}$ is an asymptotically stable fixed point as eigenvalues all have a negative real part. Consequently non-vanishing stationary solutions must necessarily be detached and appear as isolated branches.

The comparison between the single-mode ROM and full-order HBFEM solution, using a Fourier expansion of order 5, is reported in Fig. 6(a). The case corresponding to the highest value of $\kappa$ is also reported in a separate chart in Fig. 6(b) focusing on the low amplitude region. The first remark is the perfect agreement between full order solutions and reduced model at any forcing value. Furthermore, the expected isolated solutions predicted from theory are recovered in both reduced and full model. Only a very small discrepancy between full order and reduced model is observed for $\kappa=350 \mathrm{~m} / \mathrm{s}^{2}$. With respect to the nondimensional quantity, this leads to $\check{\kappa}=0.034$ : a larger value as compared to the one reached in the previous loading scenario, meaning that order $\varepsilon^{2}$ terms can still be safely neglected. About computational performance, they are here identical to those obtained in the previous example, which is logical, being the amplitude of the response comparable and the total number of computed FRCs identical.

a)

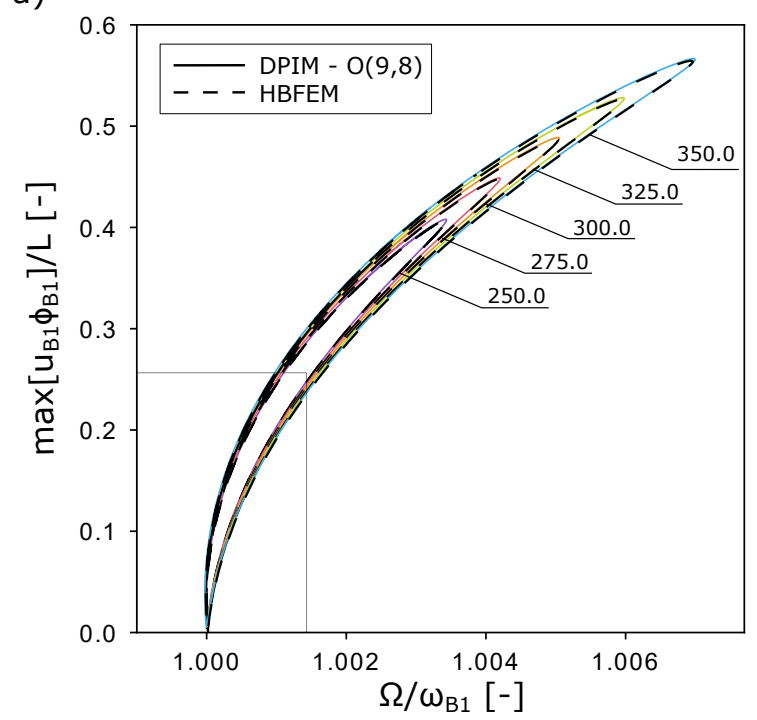

b)

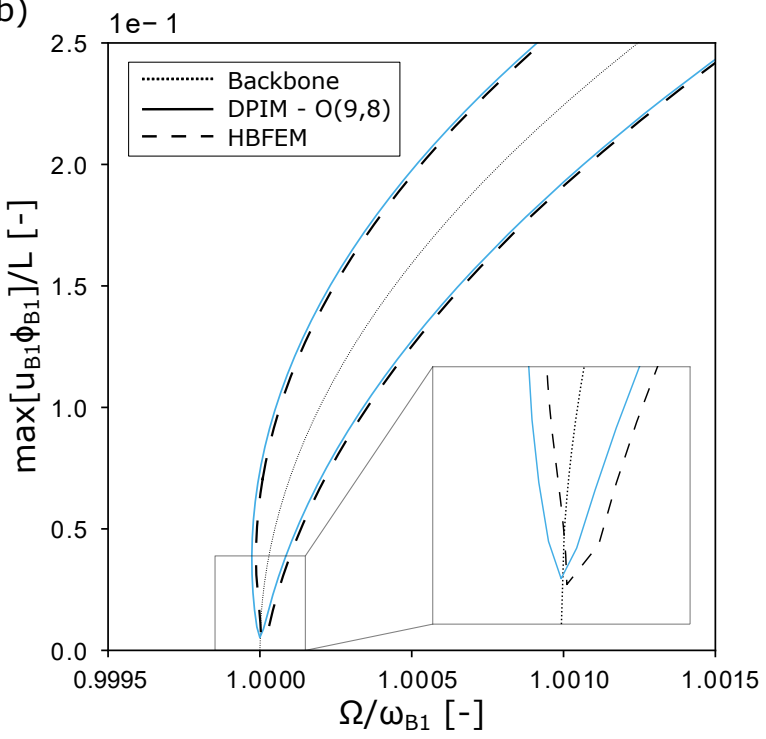

Fig. 6 Cantilever beam and isolated frequency response curves obtained by forcing at $\Omega$ equal to the frequency of the first bending mode and with a force field distribution proportional to the second bending mode. (a) shows the comparison between reduced model and HBFEM simulations for five different load multiplier values. $\kappa$ values are reported as tags in the chart. In increasing order, the dimensionless loading multiplier $\check{\kappa}$ as defined in Eq. 103 is equal to $2.43 \cdot 10^{-2}, 2.67 \cdot 10^{-2}$, $2.91 \cdot 10^{-2}, 3.16 \cdot 10^{-2}$, and $3.40 \cdot 10^{-2}$. (b) zoomed details the FRC obtained for the highest forcing value, i.e. for $\kappa=350$ $\mathrm{m} / \mathrm{s}^{2}$. The backbone curve of the system is also reported to highlight that the predicted isola bifurcates from it.

As a final remark, let us underline that the existence of these isolas is directly determined by the distribution of the forcing field. Indeed, in this example strong modal coupling is present between first and second bending modes, the latter corresponding to the distribution of the forcing acting on the system. This strong cubic coupling is reflected by the second-order non-autonomous coefficients retrieved in the reduced dynamics, which in turn provide the driving force for the appearance of isolated solutions in the frequency response curves. From the generic expressions reported in Appendix E second-order non-autonomous reduced dynamics coefficients have the following expression, here reported for a single monomial:

$$
\hat{f}_{+, 1\left\{11^{*}\right\}}^{(2)}=\frac{\boldsymbol{\Phi}_{B_{1}}^{\mathrm{T}}}{\lambda_{1}-\lambda_{1^{*}}}\left[2 \boldsymbol{G}\left(\boldsymbol{\Psi}_{\left\{11^{*}\right\}}^{(2)}, \hat{\boldsymbol{\Psi}}_{+}^{(0)}\right)+2 \boldsymbol{G}\left(\boldsymbol{\Phi}_{B_{1}}, \hat{\mathbf{\Psi}}_{+,\left\{1^{*}\right\}}^{(1)}\right)+3 \boldsymbol{H}\left(\boldsymbol{\Phi}_{B_{1}}, \boldsymbol{\Phi}_{B_{1}}, \hat{\mathbf{\Psi}}_{+}^{(0)}\right)\right]
$$

where the dominant contribution is expressed by the cubic coupling between the first and second bending modes:

$$
\boldsymbol{\Phi}_{B_{1}}^{\mathrm{T}} \boldsymbol{H}\left(\boldsymbol{\Phi}_{B_{1}}, \boldsymbol{\Phi}_{B_{1}}, \hat{\Psi}_{+}^{(0)}\right) \propto \boldsymbol{\Phi}_{B_{1}}^{\mathrm{T}} \boldsymbol{H}\left(\boldsymbol{\Phi}_{B_{1}}, \boldsymbol{\Phi}_{B_{1}}, \boldsymbol{\Phi}_{B_{2}}\right) .
$$

We now turn to the case of an excitation frequency which is in the vicinity of twice the eigenfrequency of a mode, following the discussion led in Section 4, and more specifically to the case $\gamma=2$ addressed in Section 4.3 


\subsection{Parametric instability of a cantilever beam}

Systems are said to be parametrically excited if the excitation induces the appearance of time-varying coefficients in the equations of motion. Examples of parametric resonance date to the work by Faraday 66 and the first model was introduced by Mathieu [67]. In structural mechanics, several vibratory systems exhibit parametric excitation, as shown by the large amount of works devoted to the topic, see e.g. 68, 56, 69.70 to cite only a few. One of the most typical examples is represented by axially loaded structures (rods or plates). Inspired by this case, we here report the analysis of a parametrically excited cantilever beam and we provide insight into how parametric excitation is embedded within the presented methodology.

To this purpose, the same cantilever beam addressed in Section 6.1 is considered. Parametric excitation from experiments is observed when the structure is axially excited with a forcing frequency that is twice the natural frequency of the driven mode. Following this condition, we here propose the following idealized model:

$$
\int_{\mathrm{B}} \rho \ddot{\boldsymbol{u}} \cdot \boldsymbol{w} d \mathrm{~B}+\int_{\mathrm{B}} \mathbf{P}: \nabla^{T} \boldsymbol{w} d \mathrm{~B}=\kappa\left[\int_{\mathrm{B}} \rho \boldsymbol{\Phi}_{A_{1}} \cdot \boldsymbol{w} d \mathrm{~B}\right] \cos (\Omega t), \quad \forall \boldsymbol{w} \in \mathcal{C}(\mathbf{0}) .
$$

The forcing is now assumed to have a spatial dependence corresponding to the first axial mode of the beam which is here denoted $\boldsymbol{\Phi}_{A_{1}}$ and is illustrated in Fig. 3(c). Note that for practical applications, a pointwise forcing in the axial direction is generally considered. This more realistic case however shares with our loading scenario the assumption of having a spatial dependence orthogonal to the driven mode. Upon discretisation of Eq. (109), the following problem is obtained:

$$
\mathbf{M U ̈}+\mathbf{C U}+\mathbf{K U}+\boldsymbol{G}(\mathbf{U}, \mathbf{U})+\boldsymbol{H}(\mathbf{U}, \mathbf{U}, \mathbf{U})=\frac{1}{2} \kappa \mathbf{M} \boldsymbol{\Phi}_{A_{1}}\left(e^{+\mathrm{i} \Omega t}+e^{-\mathrm{i} \Omega t}\right),
$$

where the excitation frequency is selected as $\Omega \approx 2 \omega_{B_{1}}$, i.e. in the vicinity of twice the fundamental eigenfrequency.

Following the analysis of Section 4.3 on the new trivial resonances that have to be considered when $\Omega \approx 2 \omega_{B_{1}}$, i.e. $\gamma=2$, the reduced dynamics is now inspected in order to understand how the parametric resonance is correctly handled by the ROM. By focusing on the terms brought by the non-autonomous part only, and using a complex normal form style, the only monomials arising in the reduced dynamics are those associated to the new trivial resonances:

$$
\begin{aligned}
& \hat{\lambda}_{+}+\lambda_{1^{*}} \approx \lambda_{1}, \\
& \hat{\lambda}_{-}+\lambda_{1} \approx \lambda_{1^{*}},
\end{aligned}
$$

using the usual notation $\hat{\lambda}_{+}=+i \Omega, \hat{\lambda}_{-}=-i \Omega$ for the forcing; and $\lambda_{1} \approx i \omega_{B_{1}}, \lambda_{1^{*}} \approx-i \omega_{B_{1}}$ (under the assumption of small damping). As a consequence, the reduced dynamics along the embedding then reads:

$$
\left[\begin{array}{l}
\dot{\mathbf{z}}_{1} \\
\dot{\mathrm{z}}_{1^{*}}
\end{array}\right]=\boldsymbol{f}(\mathbf{z})+\kappa\left(\left[\begin{array}{l}
\hat{f}_{+, 1\left\{1^{*}\right\}}^{(1)} \\
0
\end{array}\right] e^{+i \Omega t} \mathrm{z}_{1^{*}}+\left[\begin{array}{l}
0 \\
\hat{f}_{-, 1^{*}\{1\}}^{(1)}
\end{array}\right] e^{-i \Omega t} \mathrm{z}_{1}\right)+O\left(\left\|\mathbf{z}^{2}\right\|\right) .
$$

which highlights that the new trivial resonance conditions provide first-order terms in the non-autonomous part of the reduced dynamics, having the structure required to convey the parametric excitation. In order to push further the analysis, let us make explicit how the different orders are connected both to the forcing and to the quadratic nonlinearity. In particular, the coefficients $\hat{\mathbf{f}}^{(1)}$ need to be more detailed and related to the structural characteristics. Also, the strong quadratic coupling existing between axial and flexural modes in planar structures is key to allow activation of the trivial resonance, as it will be associated to the resonant monomial.

From the perspective of the invariance equation, for $\Omega \approx 2 \omega_{B_{1}}$, no resonance conditions are observed at order zero. Therefore, the zero-order non-autonomous homological equations read:

$$
\begin{aligned}
{\left[\hat{\lambda}_{+}^{2} \mathbf{M}+\hat{\lambda}_{+} \mathbf{C}+\mathbf{K}\right] \hat{\mathbf{\Psi}}_{+}^{(0)}=\hat{\mathbf{F}}_{+}=\kappa \frac{1}{2} \mathbf{M} \boldsymbol{\Phi}_{A_{1}}, } \\
{\left[\hat{\lambda}_{-}^{2} \mathbf{M}+\hat{\lambda}_{-} \mathbf{C}+\mathbf{K}\right] \hat{\Psi}_{-}^{(0)}=\hat{\mathbf{F}}_{-}=\kappa \frac{1}{2} \mathbf{M} \boldsymbol{\Phi}_{A_{1}} . }
\end{aligned}
$$

Recalling that $\kappa \mathbf{M} \boldsymbol{\Phi}_{A_{1}}$ is the forcing vector, see Eq. 110 , one observes that the zero-order non-autonomous mapping corresponds to the linear response of the system, a result which is here equivalent to the one already analyzed in Section 4.1, see e.g. Eq. (72).

Moving to the first order non-autonomous homological equations, we now have the resonance conditions introduced by Eq. (111). To avoid singularity of the resulting homological equations we need to impose proper orthogonality conditions, leading to: 


$$
\begin{array}{r}
{\left[\left(\hat{\lambda}_{+}+\lambda_{1}\right)^{2} \mathbf{M}+\left(\hat{\lambda}_{+}+\lambda_{1}\right) \mathbf{C}+\mathbf{K}\right] \hat{\mathbf{\Psi}}_{+,\{1\}}^{(1)}=-2 \mathbf{G}\left(\boldsymbol{\Phi}_{B_{1}}, \hat{\mathbf{\Psi}}_{+}^{(0)}\right)} \\
{\left[\left(\hat{\lambda}_{+}+\lambda_{1^{*}}\right)^{2} \mathbf{M}+\left(\hat{\lambda}_{+}+\lambda_{1^{*}}\right) \mathbf{C}+\mathbf{K}\right] \hat{\mathbf{\Psi}}_{+,\left\{1^{*}\right\}}^{(1)}+\left(\hat{\lambda}_{+}+\lambda_{1^{*}}\right) \mathbf{M} \boldsymbol{\Phi}_{B_{1}} \hat{f}_{+, 1\left\{1^{*}\right\}}^{(1)}=-2 \mathbf{G}\left(\boldsymbol{\Phi}_{B_{1}}, \hat{\mathbf{\Psi}}_{+}^{(0)}\right)} \\
{\left[\left(\hat{\lambda}_{-}+\lambda_{1}\right)^{2} \mathbf{M}+\left(\hat{\lambda}_{-}+\lambda_{1}\right) \mathbf{C}+\mathbf{K}\right] \hat{\mathbf{\Psi}}_{-,\{1\}}^{(1)}+\left(\hat{\lambda}_{-}+\lambda\right) \mathbf{M} \boldsymbol{\Phi}_{B_{1}} \hat{f}_{-, 1^{*}\{1\}}^{(1)}=-2 \mathbf{G}\left(\boldsymbol{\Phi}_{B_{1}}, \hat{\mathbf{\Psi}}_{-}^{(0)}\right)} \\
{\left[\left(\hat{\lambda}_{-}+\lambda_{1^{*}}\right)^{2} \mathbf{M}+\left(\hat{\lambda}_{-}+\lambda_{1^{*}}\right) \mathbf{C}+\mathbf{K}\right] \hat{\mathbf{\Psi}}_{-,\left\{1^{*}\right\}}^{(1)}=-2 \mathbf{G}\left(\boldsymbol{\Phi}_{B_{1}}, \hat{\mathbf{\Psi}}_{-}^{(0)}\right),}
\end{array}
$$

and as a consequence, following the derivation reported in Appendix E the reduced dynamics coefficients for a complex normal form style parametrisation read:

$$
\begin{aligned}
& \hat{f}_{+, 1\left\{1^{*}\right\}}^{(1)}=\frac{\boldsymbol{\Phi}_{B_{1}}^{\mathrm{T}}}{\lambda_{1}-\lambda_{1^{*}}}\left[-2 \boldsymbol{G}\left(\boldsymbol{\Phi}_{B_{1}}, \hat{\Psi}_{+}^{(0)}\right)\right], \\
& \hat{f}_{-, 1^{*}\{1\}}^{(1)}=\frac{\boldsymbol{\Phi}_{B_{1}}^{\mathrm{T}}}{\lambda_{1^{*}}-\lambda_{1}}\left[-2 \boldsymbol{G}\left(\boldsymbol{\Phi}_{B_{1}}, \hat{\Psi}_{-}^{(0)}\right)\right] .
\end{aligned}
$$

The above equations highlight how the reduced dynamics coefficients that induce parametric excitation are proportional to the quadratic coupling between the master modal coordinate $\boldsymbol{\Phi}_{B_{1}}$ and the zero-order non-autonomous mapping, as expressed by the right-hand sides of Eqs. (114). As evidenced by Eqs. (113), zero-order non-autonomous mappings are linearly proportional to the applied forcing, i.e. it is proportional to $\boldsymbol{\Phi}_{A_{1}}$. As a consequence the following proportionality holds:

$$
\boldsymbol{\Phi}_{B_{1}}^{\mathrm{T}} \boldsymbol{G}\left(\boldsymbol{\Phi}_{B_{1}}, \hat{\mathbf{\Psi}}_{ \pm}^{(0)}\right) \propto \boldsymbol{\Phi}_{B_{1}}^{\mathrm{T}} \boldsymbol{G}\left(\boldsymbol{\Phi}_{B_{1}}, \boldsymbol{\Phi}_{A_{1}}\right) .
$$

Again, the modal quadratic coupling coefficient $\boldsymbol{\Phi}_{B_{1}}^{\mathrm{T}} \mathbf{G}\left(\boldsymbol{\Phi}_{B_{1}}, \boldsymbol{\Phi}_{A_{1}}\right)$ appears, a term that is known to have large values for such beam, see e.g. [71,8. Consequently a forcing with high modal projection value onto the axial mode would prove excellent to parametrically excite the system.

All in all, the coefficient of the reduced dynamics $\hat{\mathbf{f}}^{(1)}$, that drives the solutions through trivially resonant monomials, appears thus as a product, through the quadratic nonlinearity $\mathbf{G}$, of forcing and master mode. In the present example, i.e. a flat cantilever, a forcing proportional to the master mode would not be able to parametrically excite the system, since the equations of the bending modes are free of quadratic nonlinearity (planar symmetric beam) and $\mathbf{G}\left(\boldsymbol{\Phi}_{B_{1}}, \boldsymbol{\Phi}_{B_{1}}\right)$ vanishes.

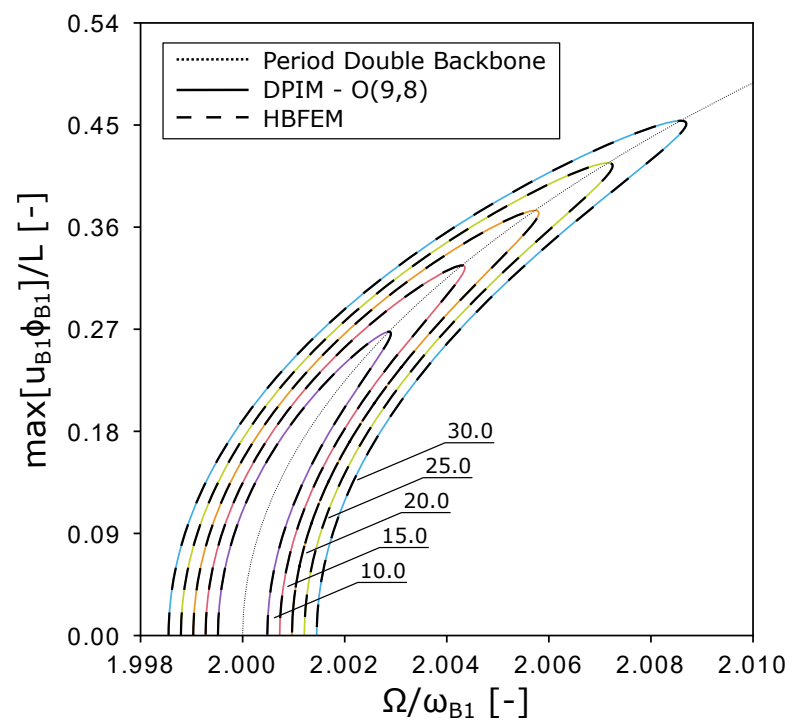

Fig. 7 Cantilever beam and parametric excitation. Comparison between HBFEM simulations and DPIM reduced model obtained with order 9 expansion of the autonomous part and order 8 expansion of the non-autonomous part. Parametrization is performed in complex normal form style. Values of the load multiplier $\kappa\left[\mathrm{m} / \mathrm{s}^{2}\right]$ values are reported as tags in the chart. In increasing order, the dimensionless loading multiplier $\check{\kappa}$ is equal to $9.33 \cdot 10^{-4}, 1.4 \cdot 10^{-3}, 1.87 \cdot 10^{-3}, 2.33 \cdot 10^{-3}$, and $2.8 \cdot 10^{-3}$. The backbone of the system is reported as a function of the continuation parameter $\Omega$. The backbone describes an oscillatory motion with period equal to twice that of the continuation parameter. 
Let us now illustrate how these theoretical findings translate in the numerical results. For this example, the Rayleigh damping is selected as stiffness proportional with $\mathbf{C}=\omega_{B_{1}} /\left(2.5 \cdot 10^{10}\right) \mathbf{K}$. This choice has been used mainly because stiffness proportional damping heavily dampens the high-frequency slave modes, hence their effect is observed only in the nonlinear terms of the reduced dynamics. The structure is excited at $\Omega \approx 2 \omega_{B_{1}}$ for $\kappa$ values equal to $10,15,20,25$, and $30 \mathrm{~m} / \mathrm{s}^{2}$. Validation is again performed with HBFEM simulations on the complete structure. The Fourier expansion of full-order simulations is chosen equal to 7 . The ROM is obtained by parametrising the system motion along the first bending mode with an asymptotic expansion of order 9 for the autonomous part and of order 8 for the non-autonomous part in complex normal form style. The ROM has been computed for a single excitation frequency, here selected as $\Omega=2 \omega_{B_{1}}$.

The comparison between full-order and reduced-order model solutions is reported in Fig. 7, where a perfect agreement is observed. The perfect agreement is further justified by the small value of $\breve{\kappa}$, which is equal to $2.8 \cdot 10^{-3}$ at most. The results highlight the main features of the parametric instability [68, 56]. Bifurcated branches with non-zero amplitudes appear at period doubling bifurcation points. As expected for nonlinear parametrically excited systems, the frequency range enclosed between the two period doubling bifurcations increases with the forcing amplitude. An important remark is that the zero amplitude of the modal amplitude $\mathrm{u}_{B_{1}}$ outside the region of parametric instability is observed only because the forcing is orthogonal to the linear master subspace, hence zero order mappings are orthogonal as well. The reduction method can however deal with more complex scenario where for example primary resonance might be entangled with parametric instability, but this is left for future works.

In this Section we showed how the presented approach can be exploited to model parametrically excited systems and we applied it to model a cantilever beam subjected to axial loading. Results highlighted that the predictions offered by this technique do not only provide qualitative estimations of nonlinear dynamics phenomena of structures, but also excellent quantitative agreement.

6.4 Shallow arch subjected to a generic force

a)

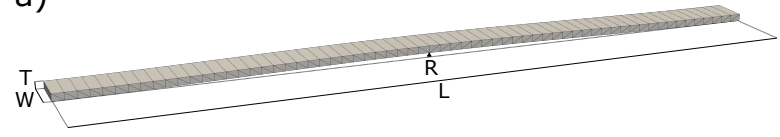

b)

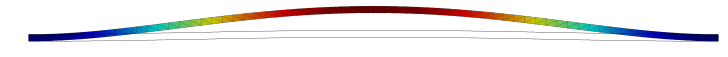

C)

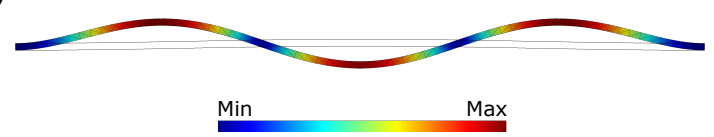

Fig. 8 Shallow arch. (a) Geometrical parameters: $\mathrm{L}=640 \mu \mathrm{m}, \mathrm{R}=3.84 \mu \mathrm{m}, \mathrm{W}=32 \mu \mathrm{m}, \mathrm{T}=6.4 \mu \mathrm{m}$. (b) Displacement field $\boldsymbol{\Phi}_{B_{1}}$ of the first bending mode; (c) Displacement field $\boldsymbol{\Phi}_{B_{3}}$ of the third bending mode;

In Sections 6.1 6.2 and 6.3 we applied the proposed method to model systems excited at their main resonance and to parametrically excited systems. The applied loads were ideal, and in general realistic forcing acting on the system will not be co-linear with any of the modes of the structure. While an industrial application of the presented method is left to Section 6.5. here we consider an intermediate case, that is a structure subjected to a forcing obtained as a linear combination of two modes. This serves as a guided example towards more realistic applications, since in a general setting we can split the forcing in its contributions to the master and to the slave coordinates, respectively.

The structure under consideration is the clamped-clamped arch reported in Fig. 8(a). It is a sine-arched structure with a length of $640 \mu \mathrm{m}$, rise height of $3.84 \mu \mathrm{m}$, thickness of $6.4 \mu \mathrm{m}$, and width of $32 \mu \mathrm{m}$. Material properties are those of polycrystalline silicon, which is modeled as isotropic with a Young's modulus of 160 GPa, Poisson's ratio of 0.22 , and a density of $2320 \mathrm{~kg} / \mathrm{m}^{3}$. The structure is actuated near the frequency of its first symmetric bending mode $\boldsymbol{\Phi}_{B_{1}}$, i.e. $1.03 \mathrm{rad} / \mu \mathrm{s}$. The displacement field associated to this mode is depicted in Fig. 8(b). The forcing has a spatial distribution proportional to a linear combination of this 

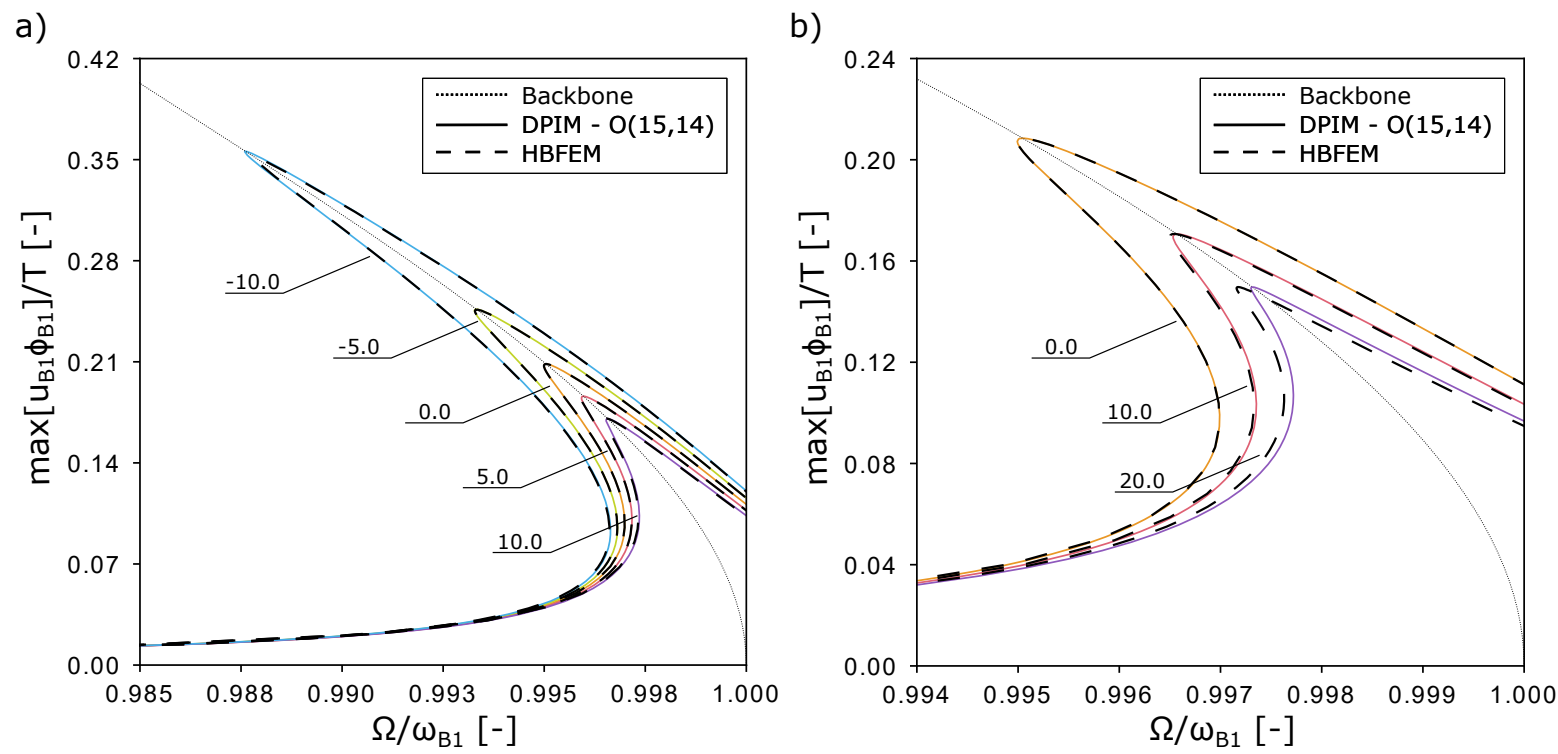

Fig. 9 Shallow arch. (a) Comparison between FRCs obtained from the reduced model and FRCs computed from HBFEM simulations. The autonomous part is expanded to order 15 , and the non-autonomous part to order $14 . \kappa_{1}$ value is constant and equal to $0.03 \mu \mathrm{m} / \mu \mathrm{s}^{2} . \kappa_{2}$ values, reported as tags in the chart, are $-10,-5,0,5,10 \mu \mathrm{m} / \mu \mathrm{s}^{2}$. They correspond to dimensionless $\check{\kappa}$ values of $-0.135,-0.0677,0,0.0677$, and 0.135 , respectively. (b) Failure of the method for high forcing values. Full order solutions progressively differ from reduced model solutions upon an increase of the $\kappa_{2}$ value. In this chart, the $\check{\kappa}$ value computed for $\kappa_{2}$ imposed as 0,10 , and $20 \mu \mathrm{m} / \mu \mathrm{s}^{2}$ is equal to $0,0.135$, and 2.271 .

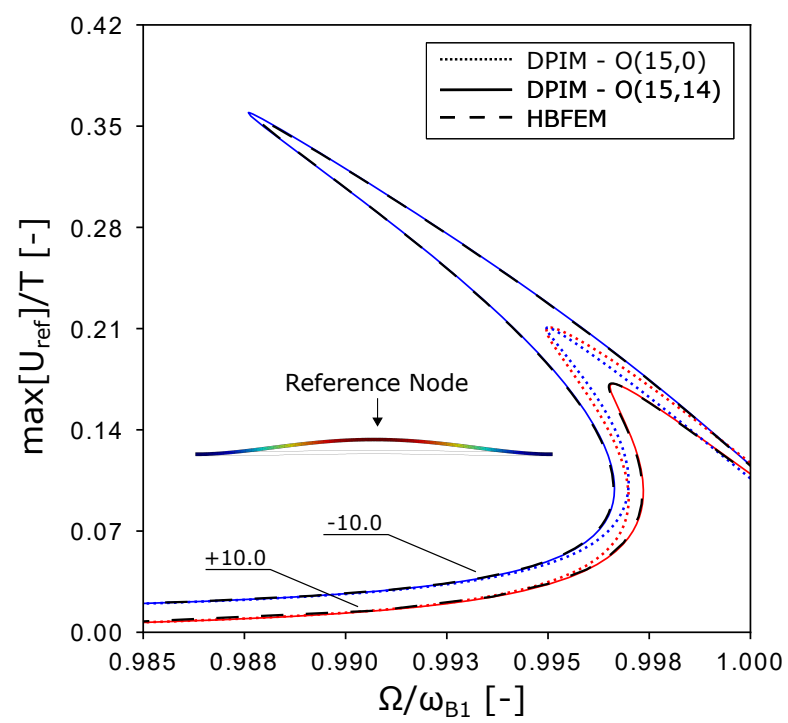

Fig. 10 Shallow arch. FRCs for the displacement node highlighted in the chart for different values of the load multiplier $\kappa_{2}$, i.e. the harmonic force contribution proportional to a slave mode. $\kappa_{1}$ is fixed at $0.03 \mu \mathrm{m} / \mu \mathrm{s}^{2}$, while $\kappa_{2}$ is taken equal to +10 and $-10 \mu \mathrm{m} / \mu \mathrm{s}^{2}$, respectively. Data obtained from the reduced model are compared with full order HBFEM simulations.

mode and of the second symmetric bending mode $\boldsymbol{\Phi}_{B_{3}}$ associated to the displacement field reported in Fig. 8 (c). The corresponding weak formulation of the problem is:

$$
\int_{\mathrm{B}} \rho \ddot{\boldsymbol{u}} \cdot \boldsymbol{w} d \mathrm{~B}+\int_{\mathrm{B}} \mathbf{P}: \nabla^{T} \boldsymbol{w} d \mathrm{~B}=\left[\int_{\mathrm{B}} \rho\left(\kappa_{1} \boldsymbol{\Phi}_{B_{1}}+\kappa_{2} \boldsymbol{\Phi}_{B_{3}}\right) \cdot \boldsymbol{w} d \mathrm{~B}\right] \cos (\Omega t), \quad \forall \boldsymbol{w} \in \mathcal{C}(\mathbf{0}),
$$

where $\kappa_{1}$ and $\kappa_{2}$ are scalar load multipliers and $\Omega \approx \omega_{B_{1}}$. Mode $\boldsymbol{\Phi}_{B_{3}}$ is nonlinearly coupled to mode $\boldsymbol{\Phi}_{B_{1}}$ since they are both bending symmetric modes. The eigenfrequency of mode $\boldsymbol{\Phi}_{B_{3}}$ is $4.59 \mathrm{rad} / \mu \mathrm{s}$, thus no internal resonance is at hand with mode $\boldsymbol{\Phi}_{B_{1}}$ and the ROM can be constructed with a single master mode assumption, selecting mode 1 as master. The choice on the forcing is made to ensure that part of the forcing acting on the structure excites a mode that is nonlinearly coupled to the master, such that both the autonomous and non-autonomous parts of the reduced dynamics will be altered. The ROM is computed 
a)

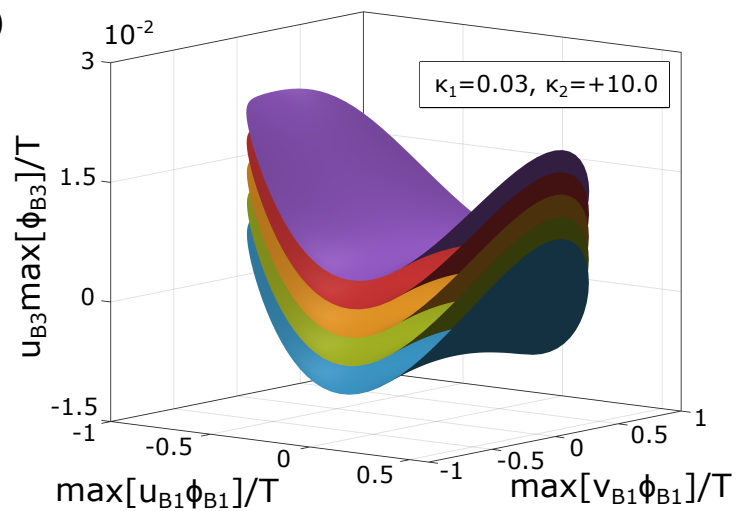

b)

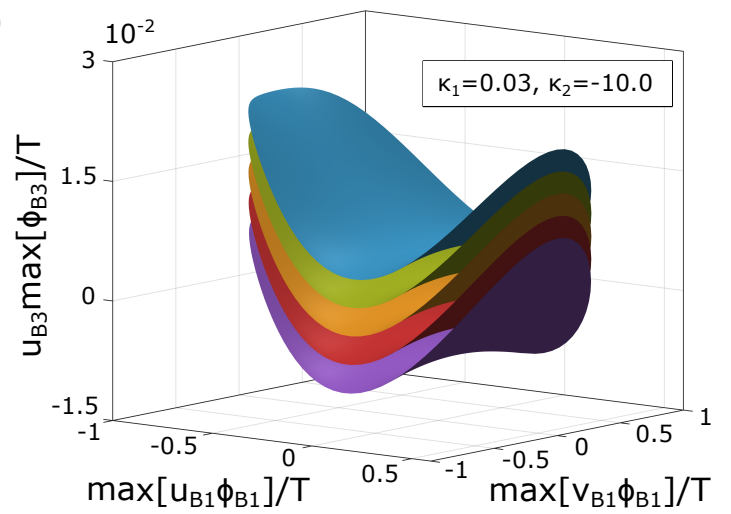

Fig. 11 Shallow arch: graphical representation of the whisker. The whisker is depicted for different phase values $\Theta=\Omega t$ along the subspace defined by modal displacement and velocity of master mode $\boldsymbol{\Phi}_{B_{1}}$, and modal displacement of mode $\boldsymbol{\Phi}_{B_{3}}$. Since its motion is periodic, oscillations for half a period only are shown. All charts are obtained for $\kappa_{1}=0.03 \mu \mathrm{m} / \mu \mathrm{s}^{2}$. Chart (a) is obtained for $\kappa_{2}=10 \mu \mathrm{m} / \mu \mathrm{s}^{2}$, while (b) reports data obtained for $\kappa_{2}=-10 \mu \mathrm{m} / \mu \mathrm{s}^{2}$. Colors correspond to the phase chosen to represent the whisker. $(\square),(\square),(\square),(\square),(\square)$ colors correspond to a phase equal to $0, \pi / 4, \pi / 2,3 \pi / 4$, and $\pi$, respectively. We highlight that the time-modulations of the whisker are dominated by zero-order oscillations.

a)

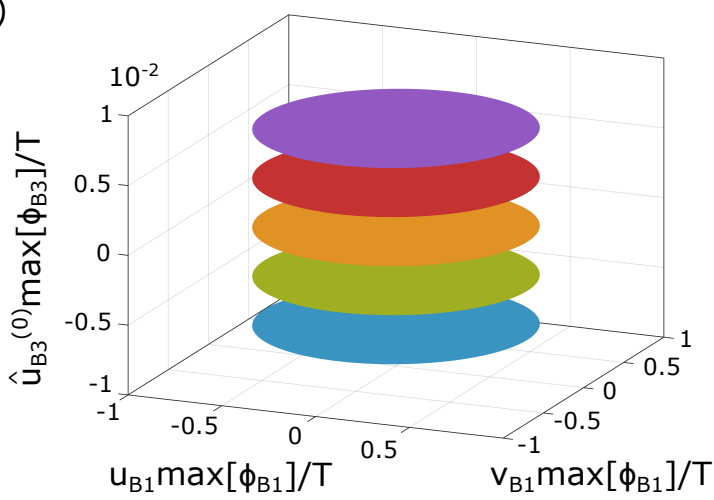

c)

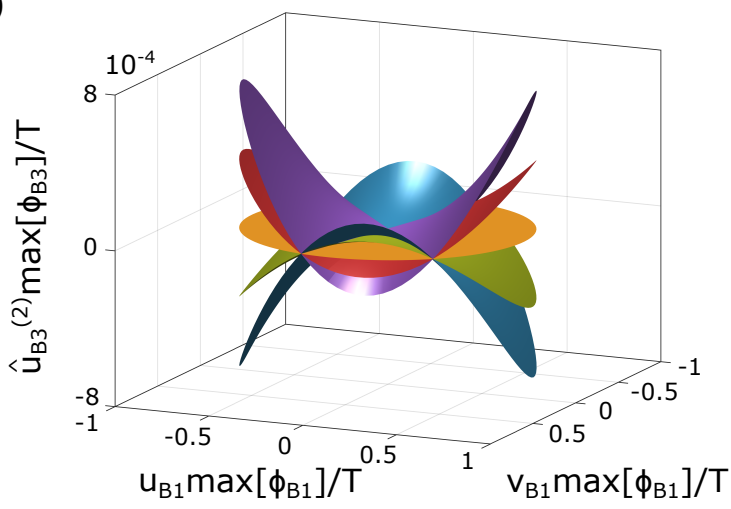

b)

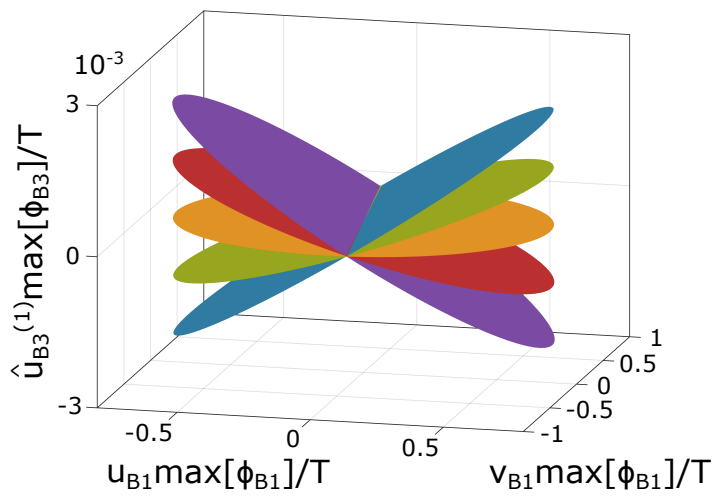

d)

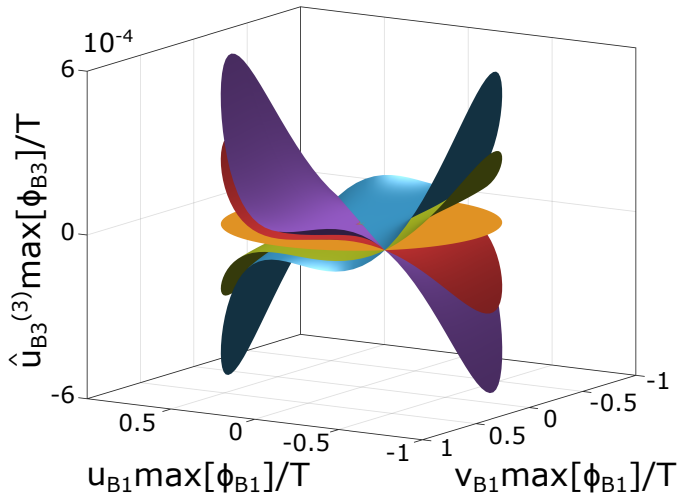

Fig. 12 Shallow arch. Contributions of the first four orders of the non-autonomous nonlinear mapping to the third bending mode $\boldsymbol{\Phi}_{B_{3}}$, versus the master mode displacement and velocity. The charts are computed by summing the contributions of all combinations $\mathcal{I}$ of a given order $p$ of the non-autonomous expansion as also done for the schematic representation reported in Fig. 2 The deformations of the whisker during the oscillation is evidenced by filtering out the main contribution given by order zero. The order $p$ represented in the charts is equal to 0 (a), 1 (b), 2 (c), and 3 (c). Functions are represented for different values of the phase $\Theta=\Omega t .(\square),(\square),(\square),(\square),(\square)$ colors correspond to phases $0, \pi / 4, \pi / 2,3 \pi / 4$, and $\pi$, respectively. 
for the excitation frequency $\Omega=\omega_{B_{1}}$. The full system is built upon addition of damping and finite element discretisation of the arch using 15-nodes quadratic wedge elements, yielding a system of 3483 degrees of freedom. The semi-discrete governing equation reads:

$$
\mathbf{M} \ddot{\mathbf{U}}+\mathbf{C} \dot{\mathbf{U}}+\mathbf{K U}+\boldsymbol{G}(\mathbf{U}, \mathbf{U})+\boldsymbol{H}(\mathbf{U}, \mathbf{U}, \mathbf{U})=\frac{1}{2}\left(\kappa_{1} \mathbf{M} \boldsymbol{\Phi}_{B_{1}}+\kappa_{2} \mathbf{M} \boldsymbol{\Phi}_{B_{3}}\right)\left(e^{+i \Omega t}+e^{-i \Omega t}\right),
$$

where $\mathbf{C}$ is taken equal to $\left(\omega_{B_{1}} / 500\right) \mathbf{M}$. The system response is analysed by varying $\kappa_{1}$ and $\kappa_{2}$ parameters. Importantly, only $\kappa_{1}$ intervenes in the non-autonomous part of the reduced dynamics of the system at order 0. Indeed, as shown in the previous Sections, at this order only the portion of the forcing which is co-linear with the master modal subspace affects the reduced dynamics. Therefore, by truncating the asymptotic expansion of the non-autonomous part at order 0 , one would not observe any dependence of the reduced dynamics $\hat{\boldsymbol{f}}(\mathbf{z}, \boldsymbol{\Omega}, t)$ on $\kappa_{2}$.

The system response is first studied for a fixed value of $\kappa_{1}$, and by varying $\kappa_{2}$. In this analysis $\kappa_{1}$ is set to $0.03 \mu \mathrm{m} / \mu \mathrm{s}^{2}$, while $\kappa_{2}$ spans the values $-10,-5,0,5,10$, and $20 \mu \mathrm{m} / \mu \mathrm{s}^{2}$. The choice of using both negative and positive values is dictated by the cubic coupling between the two symmetric modes, which is therefore sensitive to the phase of the applied forcing. As for the previous examples, results are compared with HBFEM simulations of the full-order model using a Fourier expansion order equal to 7. A converged ROM, obtained with an asymptotic expansion of the autonomous part of order 15, and an asymptotic expansion of the non autonomous part of order 14, is compared to the reference. A noteworthy comment on this analysis is that the forcing has a modal participation factor highly unbalanced towards the slave mode, with $\kappa_{2} \gg \kappa_{1}$. However, even with such difference, the choice of the master mode for the reduction step is dictated by the resonance conditions between the eigenvalues of the forcing and those of the autonomous system, which are verified for the fundamental mode $\boldsymbol{\Phi}_{B_{1}}$.

Numerical results obtained for these load multipliers are reported in Fig. 9. First of all, we stress the remarkable agreement between full-order and reduced-order model simulations, which is the main feature of the presented approach, together with remarkable speed ups. Indeed the HBFEM analyses for the full order problem required 1 hour for each FRC, while the same curve is obtained in less than 5 minutes from the ROM. Moving to more technical details of the reported data, we highlight that changing the forcing value acting on a slave mode has profound consequences on the nonlinear dynamical response of the structure both in terms of folding frequency and maximum oscillation amplitudes. This effect does not scale linearly with the load multiplier acting on the slave mode, underlining that a parametric-like amplification of the structure is developing due to the presence of high order non-autonomous coefficients in the reduced dynamics. The accuracy of the ROM degrades by increasing the forcing amplitude. This is obvious in Fig. 9(b), which reports a departure from full-order simulations when the $\kappa_{2}$ value approaches the value of $20 \mu \mathrm{m} / \mu \mathrm{s}^{2}$. The failure is caused by the violation of the assumption of small forcing. Indeed, the load multipliers can be rescaled according to the main parameters of the model as provided by Eq. (103). Being $\operatorname{Max}\left[\boldsymbol{\Phi}_{B_{1}}\right]=0.090$, and by considering in this case as characteristic displacement the thickness $T$ of the arch, the resulting value of $\check{\kappa}$ is 0.271 , which is now closer to unity. Note that $\check{\kappa}$ has been computed for $\kappa_{2}$ only, since it is orders of magnitude larger than $\kappa_{1}$. It is worth underlining that the ROM solutions have been here computed with a high order accuracy, since orders $\mathcal{O}(15,14)$ have been selected. It has been verified that the solution is indeed converged at this order, consequently the discrepancy cannot be attributed to the order used for the expansions, but rather to the fact that $\varepsilon^{2}$ terms begin to have a nonnegligible importance in the dynamics. Moreover, the discrepancy cannot be attributed to the fact that the system has been parametrised for a single frequency value. Indeed, the departure between the two solutions has the same magnitude at $\Omega=\omega_{B_{1}}$ for $\kappa_{2}=20 \mu \mathrm{m} / \mathrm{s}^{2}$, i.e. exactly at the point where the parametrisation is computed.

Fig. 10 shows the FRCs in the physical space, by selecting a reference node in the center of the beam to show the displacement versus the frequency. In order to reconstruct the physical displacement, the nonlinear mappings need to be computed, consequently representing this quantity embeds both effects of reduced dynamics and mappings. As highlighted in Fig. 9, a high-order expansion of the non-autonomous part is necessary to obtain converged result, otherwise the ROM would predict almost the same response at any $\kappa_{2}$ value, i.e. the response obtained for $\kappa_{2}=0 \mu \mathrm{m} / \mu \mathrm{s}^{2}$. This is again clearly evidenced in Fig. 10 where the zero-order solution for the non-autonomous part provides very inaccurate results.

Let us now illustrate how the time-dependent invariant manifold is oscillating in the phase space for this specific example. Fig. 11 shows snapshots of the whisker, at different phase values $\Theta=\Omega t$. In Fig. 11.(a), the time-dependent manifold computed for $\kappa_{1}=0.03 \mu \mathrm{m} / \mu \mathrm{s}^{2}$ and $\kappa_{2}=10 \mu \mathrm{m} / \mu \mathrm{s}^{2}$ is shown, while Fig. 11.(a) reports the same case but with $\kappa_{2}=-10 \mu \mathrm{m} / \mu \mathrm{s}^{2}$. The figures clearly underline that the zero-order oscillations dominate the time-dependence of the whisker, and that a change in the forcing sign along a given slave mode reverses the phase associated to the oscillations. 
In order to get more insight and highlight the deformations of the whisker, Fig. 12 illustrates the contributions of the first four orders of expansions of the non-autonomous mapping along the third bending mode $\boldsymbol{\Phi}_{B_{3}}$. More specifically, let us introduce the following decomposition of the modal displacement:

$$
\mathrm{u}_{i}=\sum_{p=1}^{o} \mathrm{u}_{i}^{(p)}+\varepsilon \sum_{p=0}^{o-1} \hat{\mathrm{u}}_{i}^{(p)}
$$

where a generic term $\stackrel{\circ}{\mathrm{u}}_{i}^{(p)}$ collects the contributions of all mappings for a given order $p$ :

$$
\stackrel{\circ}{\mathrm{u}}_{i}^{(p)}=\boldsymbol{\Phi}_{i}^{\mathrm{T}} \mathbf{M} \sum_{\mathcal{I}} \stackrel{\circ}{\mathbf{\Psi}}_{\mathcal{I}}^{(p)} \pi_{\mathcal{I}} .
$$

Consequently, Fig. 12 refers to the contributions to the modal displacement $\mathrm{u}_{3}$ provided by the first four orders of the $\varepsilon^{1}$-homological equations. Fig. 12 (a) shows again that the main time dependence of the whisker is dominated by the up and down oscillation slaved to the external forcing and conveyed by the zero-order term. Higher order terms have smaller amplitudes, but are not zero, underlining that the whisker indeed experiences deformations. The presence of both quadratic and cubic coupling terms in arched structures is obvious from the amplitudes of the oscillations, which are of comparable magnitude at both even and odd terms of the expansion.

\subsection{Modeling of a comb-driven MEMS resonator}

In this last Section, the reduction method is applied to the analysis of a structure of industrial interest, that is a MEMS resonator [1. This class of devices serves a wealth of electronic systems since they can be exploited for sensing [72, filtering [2], and timing [73] applications. Furthermore, these systems appear to be a fertile field of application for the presented approach since fast and accurate predictions of the nonlinear dynamic response of the system are compulsory even during early design stages in order to control or exploit frequency deviations [74,75].

a)
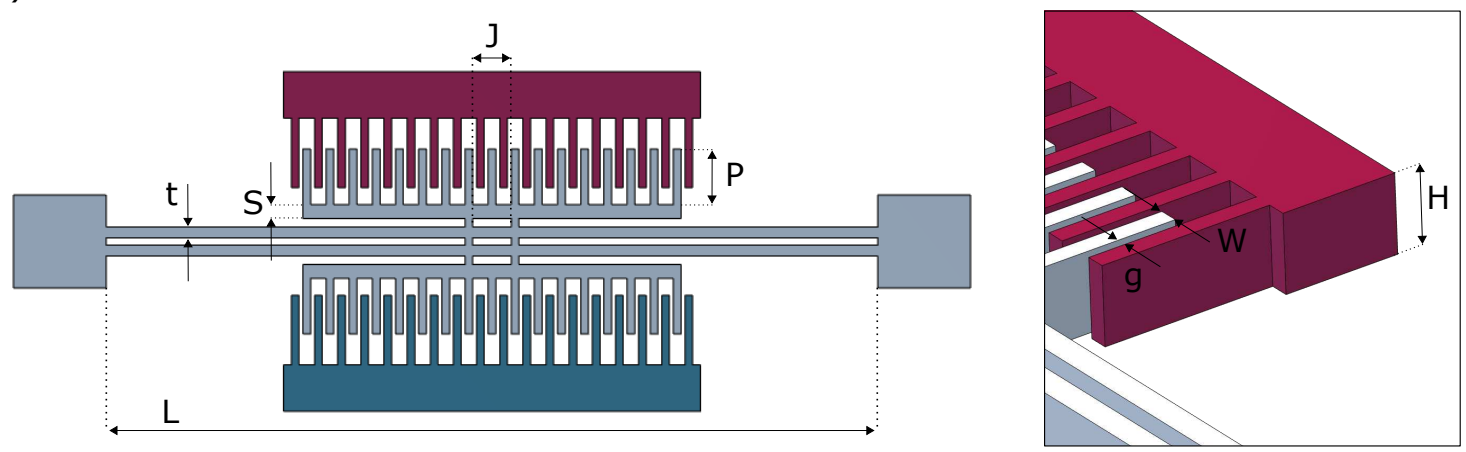

b)

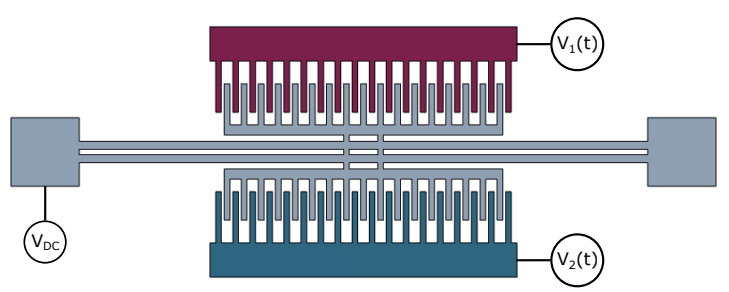

c)

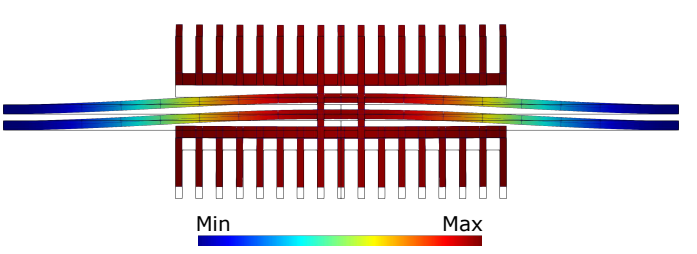

Fig. 13 (a) Geometry of the MEMS resonator. (b) Voltage bias configuration for MEMS resonator (grey), and drive electrodes (red and blue). (c) Displacement field associated to the first symmetric bending mode of the structure.

The structure under consideration is the MEMS resonator depicted in Fig. 13(a). The structure is a rework of the design initially developed by Shaw and coworkers in [76, 77]. In particular, geometrical changes are introduced to avoid internal resonances between modes, which is out of the scope of the analysis shown in this contribution.

The system consists of a clamped-clamped beam resonator actuated with comb-drive electrodes. The beam resonator is depicted in grey in Fig. 13.(a) and the two sets of electrodes required to actuate the 
structure are reported in blue and red colours. Actuation is provided by setting the voltage of the resonator to a constant bias $V_{D C}$ and by applying time-dependent voltage laws to the two sets of drive electrodes with the configuration reported in Fig. 13(b):

$$
V_{1}(t)=V_{A C} \cos (\Omega t), \quad V_{2}(t)=-V_{A C} \cos (\Omega t),
$$

where $V_{A C}$ is the amplitude of a sinusoidal voltage applied to harmonically excite the system.

Table 1 Geometrical parameters of the MEMS resonator

\begin{tabular}{|c|c|}
\hline Label & Value $[\mu \mathrm{m}]$ \\
\hline $\mathrm{L}$ & 500 \\
\hline $\mathrm{t}$ & 7 \\
\hline $\mathrm{d}$ & 5 \\
\hline $\mathrm{S}$ & 9 \\
\hline $\mathrm{P}$ & 36 \\
\hline $\mathrm{J}$ & 25 \\
\hline $\mathrm{H}$ & 20 \\
\hline $\mathrm{W}$ & 5 \\
\hline $\mathrm{g}$ & 2.5 \\
\hline
\end{tabular}

For comb-finger actuation, as the one reported in Fig. 13(a), border effects are negligible and analytic approximations for the force acting on the structure are available. In particular, the force exerted on a finger head can be expressed as

$$
\boldsymbol{t}_{e}=\frac{\epsilon_{0} \epsilon_{r} B}{d}(\Delta V(t))^{2} \boldsymbol{n}
$$

with $\Delta V(t)$ potential difference between shuttle and a given drive electrode. As a result, the force per unit normal exerted on the tips of the two sets of comb fingers of the resonator are:

$$
\begin{aligned}
\boldsymbol{t}_{e_{1}} & =\frac{\epsilon_{0} \epsilon_{r} B}{d}\left(V_{D C}+V_{A C} \cos (\Omega t)\right)^{2} \boldsymbol{n} \simeq \frac{\epsilon_{0} \epsilon_{r} B}{d}\left(V_{D C}^{2}+2 V_{D C} V_{A C} \cos (\Omega t)\right) \boldsymbol{n}, \\
\boldsymbol{t}_{e_{2}} & =\frac{\epsilon_{0} \epsilon_{r} B}{d}\left(V_{D C}-V_{A C} \cos (\Omega t)\right)^{2} \boldsymbol{n} \simeq \frac{\epsilon_{0} \epsilon_{r} B}{d}\left(V_{D C}^{2}-2 V_{D C} V_{A C} \cos (\Omega t)\right) \boldsymbol{n},
\end{aligned}
$$

where the terms in $V_{A C}^{2}$ are neglected since, as typical of these structures, $V_{A C} \ll V_{D C}$. The term proportional to $V_{D C}^{2}$ yields forces of opposite direction on the two set of combs and hence cancels out in the overall resultant. We can therefore introduce an equivalent traction vector acting on the head of the comb:

$$
\tilde{\boldsymbol{t}}_{e}=2 V_{D C} \frac{\epsilon_{0} \epsilon_{r}}{W d} \boldsymbol{n}
$$

where $W$ is the width of the comb. The traction is equal to the amplitude of the sinusoidal force provided by Eq. (123) normalised by the $V_{A C}$ component and by the area of the comb. The resulting weak formulation of the problem is then given as:

$$
\int_{\mathrm{B}} \rho \ddot{\boldsymbol{u}} \cdot \boldsymbol{w} d \mathrm{~B}+\int_{\mathrm{B}} \mathbf{P}: \nabla^{\mathrm{T}} \boldsymbol{w} d \mathrm{~B}=V_{A C}\left(\int_{\Gamma_{1}} \tilde{\boldsymbol{t}}_{e_{1}} \cdot \boldsymbol{w} d \Gamma-\int_{\Gamma_{2}} \tilde{\boldsymbol{t}}_{e_{2}} \cdot \boldsymbol{w} d \Gamma\right) \cos (\Omega t)
$$

with $\Gamma_{1}$ (resp. $\left.\Gamma_{2}\right)$ the set of comb tips exposed to the stator that is subjected to the voltage law $V_{1}(t)$ (resp. $\left.V_{2}(t)\right) . V_{A C}$ replaces the nonphysical $\kappa$ used in previous results. Equation (126) is discretised using a finite element formulation with quadratic 27 -nodes hexahedral elements. The resulting system is equal to:

$$
\mathbf{M} \ddot{\mathbf{U}}+\mathbf{C} \dot{\mathbf{U}}+\mathbf{K U}+\boldsymbol{G}(\mathbf{U}, \mathbf{U})+\boldsymbol{H}(\mathbf{U}, \mathbf{U}, \mathbf{U})=V_{A C} \frac{\hat{\mathbf{F}}}{2}\left(e^{+i \Omega t}+e^{-i \Omega t}\right),
$$

The damping matrix is selected as $\mathbf{C}=\left(\omega_{B_{1}} / 10^{4}\right) \mathbf{M}$. The MEMS is excited at its first resonant mode, corresponding to a symmetric bending mode, hence $\Omega \approx \omega_{B_{1}}$. The associated displacement field is reported in Fig. 13 To model the presented device we parametrise the system motion adopting $\boldsymbol{\Phi}_{B_{1}}$, i.e. the first symmetric bending mode, as master mode. Parametrisation is performed with complex normal form style and an expansion of order 15 for the autonomous part and order 14 for the non-autonomous part. From a mathematical point of view, the problem resembles the most practical case of the previous example, i.e. a structure subjected to main resonance excitation with a forcing that is neither perfectly co-linear nor orthogonal to the master mode. The main difference is that the loading scenario is here more physical, i.e. 


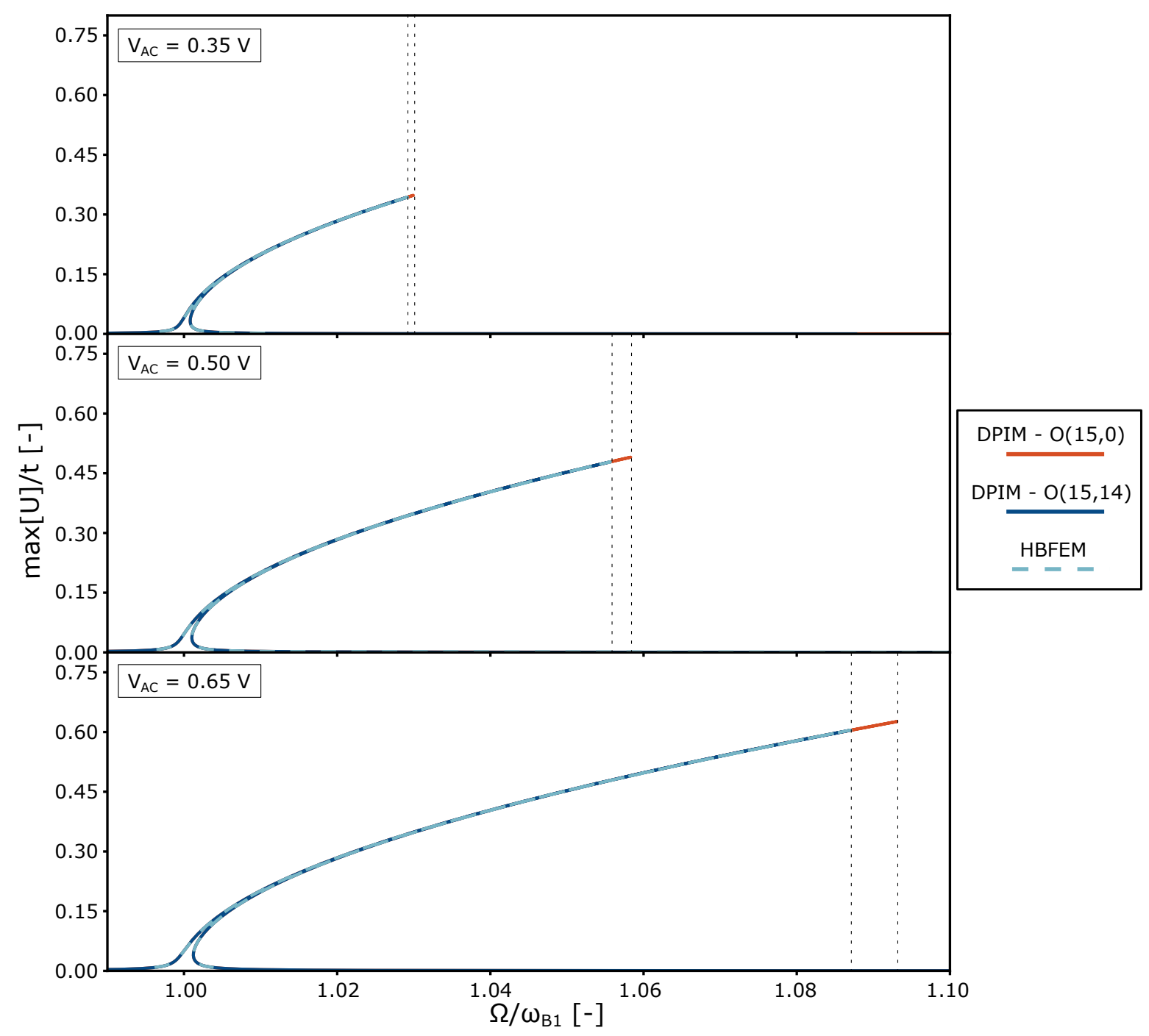

Fig. 14 Comb finger MEMS resonator. Predicted FRCs for different amplitudes of the electrical potential. Results provided by the DPIM are compared with full order HBFEM simulations. DPIM-O $(15,14)$ is perfectly overlapped with HBFEM simulations, while DPIM-O $(15,0)$ overestimates the folding frequency. The folding frequencies of HBFEM and DPIM$\mathcal{O}(15,0)$ are marked with a vertical dashed line. Displacements are normalised by the thickness $t$ of the resonator.

the modal participation factor of the force along the master mode is larger compared to that along the slave modes.

Simulations are run setting $V_{D C}=50.0 \mathrm{~V}$ and spanning three different values of $V_{A C}: 0.35,0.50$, and $0.65 \mathrm{~V}$, corresponding to typical voltage amplitudes applied in the MEMS industry. The results are compared with those of the full order HBFEM with a Fourier expansion of order 5. Fig. 14 compares the obtained results for increasing values of $V_{A C}$. One can note in particular the perfect agreement between the full-order and ROM simulations, underlining how the DPIM proves efficient for modeling complex structures as the ones required by the semiconductor industry. A comparison is also drawn out between the converged $\mathcal{O}(15,14) \mathrm{ROM}$ and the $\mathcal{O}(15,0)$ solution, in order to highlight the gain in using also a high-order parametrisation for the non-autonomous part. The difference is clearly visible on the maximum point of the FRCs, and tends to increase with the amplitude.

The computational performance represents a major asset for the method. Indeed, the original full order model features 4833 degrees of freedom and the total computational time required to extract the three FRCs was close to 32 hours. On the other hand, the solution to the reduced model takes less than 10 minutes including the reduction time, thus guaranteeing an impressive speed-up. Although the targeted application is a simplified idealization of realistic MEMS, it clearly evidences the potentialities of the approach, as the cost of full order simulations would explode with larger FEM structures, while that of the reduction technique scales almost linearly [33]. 


\section{Conclusion}

This work complements the results provided in 35] for the direct parametrisation of invariant manifolds in large dimensional nonlinear systems, with application to finite element models of structures subjected to geometric nonlinearities. While the autonomous part is detailed with a generic order development in [35], this paper focuses on handling the problem raised by adding an external forcing to the initial problem. Explicit algebraic expressions for the computation of the non-autonomous part of the reduced dynamics and tensors, that map nodal degrees of freedom of the model to an invariant based time-dependent span of the phase space, have been provided. The similarity of the structure of the homological equations in both cases has been underlined, opening the door to a fast and efficient coding elaborated from the autonomous solution. The three different parametrisation styles have also been reported for implementation in a direct computational framework.

The DPIM has been here applied to tackle problems including geometric nonlinearities, and a Rayleigh damping formulation has been adopted in order to cover most engineering applications. Also, it has been demonstrated that efficient ROMs can be obtained by computing the non-autonomous terms for a single, fixed external frequency, hence avoiding the computation of the non-autonomous part of the system at each frequency.

On the theoretical point of view, the new trivial resonances arising from the non-autonomous part have been analyzed with a single master mode assumption. In the case of main resonance, the effect of the new terms due to forcing have been shown important, and a specific case of a forcing that is orthogonal to the master mode has been used to illustrate how the method can handle the birth of isolated solutions. Also, parametric resonance can be analyzed in the present setting and the key to its observation, through a computed ROM predicting such instability, has been derived and illustrated. All these features have been illustrated on dedicated examples, underlining the capacity of the method to handle very different contexts of increasing complexity, while always offering a converged and excellent prediction for a very low computational effort. At last, the method has been applied to the analysis of a MEMS resonator subjected to comb-drive actuation. This example of remarkable industrial interest evidences the impact of the presented methodology on fields that rely on resonant mechanical systems as the semiconductor industry.

Some important comments are worth stressing. The first one concerns possible guidelines concerning the selection of the parameterisation style. While generally normal form styles have better theoretical properties, with for example no restriction related to manifold foldings, graph style might have an advantage in case of structures featuring numerous internal resonances. Also, in order to catch high order resonances the normal form style needs to be pushed at least to the order of the resonance. As observed with the second-order DNF in [32, the graph style, while keeping more non-resonant low order monomials, might show an advantage in such a case. However, this point deserves further investigation.

The second point is related to the simplification of using a single, fixed forcing frequency, in order to derive the ROM. The examples shown in this paper, and especially the last MEMS resonator, which shows a very important hardening effect, underline that the ROMs do not lose any accuracy in their predictive capabilities with such assumption. As compared to the gains obtained (versatile and efficient ROM), it is thus advised to operate in this framework, and further numerical studies might be necessary in order to well ascertain the validity limit of this assumption.

The last comment concerns the limitations of the treatment of the forcing as a perturbation, with developments up to leading order in $\varepsilon$. In this work, the validity of this assumption has been quantified by introducing a nondimensional parameter $\check{\kappa}$ related to the forcing amplitude. In the reported examples, it has been shown that whenever this parameter is much smaller than unity, the leading order treatment of the forcing at $\varepsilon^{1}$ produces extremely accurate results when coupled with higher order expansions in $\mathrm{z}$; while slight departure begins to be observed for values of $\check{\kappa}$ larger than $1 / 10$. Higher-order developments in terms of $\varepsilon$ should resolve the problem, which would also make possible to study superharmonic resonances, that are not contained in the present developments. In fact, an $m: 1$ superharmonic resonance can only be predicted by an $\varepsilon^{m}$ expansion, which will produce resonance conditions that contain $m$ multiples of the forcing eigenvalue. Automated expansion up to a generic order in $\varepsilon$ are not treated here but, given the common structure of the $\varepsilon^{0}$ and $\varepsilon^{1}$ problem discussed in the main text, such a development of the method is surely possible and will be considered in future investigations. 


\section{Funding}

The work received no additional funding.

\section{Conflict of interest}

The authors declare that they have no conflict of interest.

\section{Data availability statement}

The codes developed for the presented analyses are available upon request to the authors.

\section{References}

1. V. Zega, G. Gattere, S. Koppaka, A. Alter, G. Vukasin, A. Frangi, and T. W. Kenny. Numerical modelling of nonlinearities in MEMS resonators. Journal of Microelectromechanical Systems, 29(6):1443-1454, 2020.

2. A. Z. Hajjaj, M. A. Hafiz, and M. I. Younis. Mode coupling and nonlinear resonances of MEMS arch resonators for bandpass filters. Scientific reports, 7(1):1-7, 2017.

3. J. F. Rhoads, S. W. Shaw, K. L. Turner, and R. Baskaran. Tunable microelectromechanical filters that exploit parametric resonance. Journal of Vibration and Acoustics, 127(5):423-430, 2005.

4. M. P. Mignolet, A. Przekop, S. A. Rizzi, and S. M. Spottswood. A review of indirect/non-intrusive reduced order modeling of nonlinear geometric structures. Journal of Sound and Vibration, 332:2437-2460, 2013.

5. C. Touzé, A. Vizzaccaro, and O. Thomas. Model order reduction methods for geometrically nonlinear structures: a review of nonlinear techniques. Nonlinear Dynamics, pages 1-50, 2021.

6. M. Amabili, A. Sarkar, and M. P. Païdoussis. Reduced-order models for nonlinear vibrations of cylindrical shells via the proper orthogonal decomposition method. Journal of Fluids and Structures, 18(2):227-250, 2003.

7. M. Amabili and C. Touzé. Reduced-order models for non-linear vibrations of fluid-filled circular cylindrical shells: comparison of POD and asymptotic non-linear normal modes methods. Journal of Fluids and Structures, 23(6):885903, 2007.

8. A. Vizzaccaro, A. Givois, P. Longobardi, Y. Shen, J.-F. Deü, L. Salles, C. Touzé, and O. Thomas. Non-intrusive reduced order modelling for the dynamics of geometrically nonlinear flat structures using three-dimensional finite elements. Computational Mechanics, 66:1293-1319, 2020.

9. G. Kerschen, J.-C. Golinval, A. F. Vakakis, and L. A. Bergmann. The method of proper orthogonal decomposition for dynamical characterization and order reduction of mechanical systems: an overview. Nonlinear Dynamics, 41(1):147$169,2005$.

10. S. W. Shaw and C. Pierre. Non-linear normal modes and invariant manifolds. Journal of Sound and Vibration, 150(1):170-173, 1991.

11. S. W. Shaw and C. Pierre. Normal modes for non-linear vibratory systems. Journal of Sound and Vibration, 164(1):85$124,1993$.

12. A. Steindl and H. Troger. Methods for dimension reduction and their applications in nonlinear dynamics. International Journal of Solids and Structures, 38:2131-2147, 2001.

13. C. Touzé, O. Thomas, and A. Chaigne. Hardening/softening behaviour in non-linear oscillations of structural systems using non-linear normal modes. Journal of Sound and Vibration, 273(1-2):77-101, 2004.

14. S. W. Shaw and C. Pierre. Normal modes of vibration for non-linear continuous systems. Journal of Sound and Vibration, 169(3):85-124, 1994.

15. A.M. Lyapunov. Problème général de la stabilité du mouvement. Annales de la faculté des sciences de Toulouse, Série 2,9:203-474, 1907.

16. A. F. Kelley. Analytic two-dimensional subcenter manifolds for systems with an integral. Pac. J. Math., 29:335-350, 1969.

17. G. I. Cirillo, A. Mauroy, L. Renson, G. Kerschen, and R. Sepulchre. A spectral characterization of nonlinear normal modes. Journal of Sound and Vibration, 377:284-301, 2016.

18. G. Haller and S. Ponsioen. Nonlinear normal modes and spectral submanifolds: existence, uniqueness and use in model reduction. Nonlinear Dynamics, 86(3):1493-1534, 2016.

19. G. Haller and S. Ponsioen. Exact model reduction by a slow-fast decomposition of nonlinear mechanical systems. Nonlinear Dynamics, 90(1):617-647, 2017.

20. E. Pesheck, C. Pierre, and S. Shaw. A new Galerkin-based approach for accurate non-linear normal modes through invariant manifolds. Journal of Sound and Vibration, 249(5):971-993, 2002.

21. D. Jiang. Nonlinear modal analysis based on invariant manifolds. Application to rotating blade systems. PhD thesis, University of Michigan, 2004.

22. C. Touzé and M. Amabili. Non-linear normal modes for damped geometrically non-linear systems: application to reduced-order modeling of harmonically forced structures. Journal of Sound and Vibration, 298(4-5):958-981, 2006.

23. E. Pesheck, N. Boivin, C. Pierre, and S. Shaw. Nonlinear modal analysis of structural systems using multi-mode invariant manifolds. Nonlinear Dynamics, 25:183-205, 2001.

24. C. E. N. Mazzilli, M. E.S. Soares, and O. G. P. Baracho Neto. Non-linear normal modes of a simply supported beam: continuous system and finite-element models. Computers \& Structures, 82(31):2683-2691, 2004.

25. X. Cabré, E. Fontich, and R. de la Llave. The parameterization method for invariant manifolds. i. manifolds associated to non-resonant subspaces. Indiana Univ. Math. J., 52(2):283-328, 2003.

26. X. Cabré, E. Fontich, and R. de la Llave. The parameterization method for invariant manifolds. ii. regularity with respect to parameters. Indiana Univ. Math. J., 52(2):329-360, 2003. 
27. X. Cabré, E. Fontich, and R. de la Llave. The parameterization method for invariant manifolds. iii. overview and applications. J. Differential Equations, 218(2):444-515, 2005.

28. A. Haro, M. Canadell, J.-L. Figueras, A. Luque, and J.-M. Mondelo. The parameterization method for invariant manifolds. From rigorous results to effective computations. Springer, Switzerland, 2016.

29. S. Ponsioen, T. Pedergnana, and G. Haller. Automated computation of autonomous spectral submanifolds for nonlinear modal analysis. Journal of Sound and Vibration, 420:269 - 295, 2018.

30. T. Breunung and G. Haller. Explicit backbone curves from spectral submanifolds of forced-damped nonlinear mechanical systems. Proceedings of the Royal Society A: Mathematical, Physical and Engineering Sciences, 474(2213):20180083, 2018 .

31. S. Jain, P. Tiso, and G. Haller. Exact nonlinear model reduction for a von Kàrmàn beam: slow-fast decomposition and spectral submanifolds. Journal of Sound and Vibration, 423:195-211, 2018.

32. A. Vizzaccaro, Y. Shen, L. Salles, J. Blahos, and C. Touzé. Direct computation of nonlinear mapping via normal form for reduced-order models of finite element nonlinear structures. Computer Methods in Applied Mechanics and Engineering, 384:113957, 2021.

33. A. Opreni, A. Vizzaccaro, C. Touzé, and A. Frangi. Model order reduction based on direct normal form: Application to large finite element MEMS structures featuring internal resonance. Nonlinear Dynamics, 105:1237-1272, 2021.

34. S. Jain and G. Haller. How to compute invariant manifolds and their reduced dynamics in high-dimensional finiteelement models? Nonlinear Dynamics, 107:1417-1450, 2022.

35. A. Vizzaccaro, A. Opreni, L. Salles, A. Frangi, and C. Touzé. High order direct parametrisation of invariant manifolds for model order reduction of finite element structures: application to large amplitude vibrations and uncovering of a folding point. arXiv, 2109.10031, 2021.

36. M. Li, S. Jain, and G. Haller. Nonlinear analysis of forced mechanical systems with internal resonance using spectral submanifolds-part i: Periodic response and forced response curve. arXiv preprint arXiv:2106.05162, 2021.

37. M. Li and G. Haller. Nonlinear analysis of forced mechanical systems with internal resonance using spectral submanifolds-part ii: Bifurcation and quasi-periodic response. arXiv preprint arXiv:2108.08152, 2021.

38. D. Jiang, C. Pierre, and S. Shaw. Nonlinear normal modes for vibratory systems under harmonic excitation. Journal of Sound and Vibration, 288(4-5):791-812, 2005.

39. A. Haro and R. de la Llave. A parameterization method for the computation of invariant tori and their whiskers in quasi-periodic maps: rigorous results. Journal of Differential Equations, 228(2):530-579, 2006.

40. A. Haro and R. de la Llave. A parameterization method for the computation of invariant tori and their whiskers in quasi-periodic maps: numerical algorithms. Discrete 8 Continuous Dynamical Systems-B, 6(6):1261, 2006.

41. A. Haro and R. de La Llave. A parameterization method for the computation of invariant tori and their whiskers in quasi-periodic maps: explorations and mechanisms for the breakdown of hyperbolicity. SIAM Journal on Applied Dynamical Systems, 6(1):142, 2007

42. S. Ponsioen, T. Pedergnana, and G. Haller. Analytic prediction of isolated forced response curves from spectral submanifolds. Nonlinear Dynamics, 98(4):2755-2773, 2019.

43. S. Ponsioen, S. Jain, and G. Haller. Model reduction to spectral submanifolds and forced-response calculation in high-dimensional mechanical systems. Journal of Sound and Vibration, 488:115640, 2020.

44. G. Holzapfel. Nonlinear solid mechanics. 2000.

45. D. F. Golla and P. C. Hughes. Dynamics of viscoelastic structures: a time-domain, Finite Element formulation. Journal of Applied Mechanics, 52(4):897-906, 1985.

46. T. Pritz. Frequency power law of material damping. Applied Acoustics, 65(11):1027-1036, 2004.

47. J.-F. Deü and D. Matignon. Simulation of fractionally damped mechanical systems by means of a Newmark-diffusive scheme. Computers 85 Mathematics with Applications, 59(5):1745-1753, 2010.

48. A. Parret-Fréaud, B. Cotté, and A. Chaigne. Time-domain damping models in structural acoustics using digital filtering. Mechanical Systems and Signal Processing, 68-69:587-607, 2016.

49. H. Sumali. Squeeze-film damping in the free molecular regime: model validation and measurement on a MEMS. Journal of Micromechanics and Microengineering, 17(11):2231, 2007.

50. C. Méndez, S. Paquay, I. Klapka, and J.-P. Raskin. Effect of geometrical nonlinearity on MEMS thermoelastic damping. Nonlinear Analysis: Real World Applications, 10(3):1579-1588, 2009

51. A. Frangi, P. Fedeli, G. Laghi, G. Langfelder, and G. Gattere. Near vacuum gas damping in MEMS: Numerical modeling and experimental validation. Journal of Microelectromechanical Systems, 25(5):890-899, 2016.

52. A. Lazarus, O. Thomas, and J.-F. Deü. Finite element reduced order models for nonlinear vibrations of piezoelectric layered beams with applications to NEMS. Finite Elements in Analysis and Design, 49:35-51, 2012.

53. C. Touzé, M. Vidrascu, and D. Chapelle. Direct finite element computation of non-linear modal coupling coefficients for reduced-order shell models. Computational Mechanics, 54(2):567-580, 2014.

54. C. Touzé. Normal form theory and nonlinear normal modes: theoretical settings and applications. In G. Kerschen, editor, Modal Analysis of nonlinear Mechanical Systems, pages 75-160, New York, NY, 2014. Springer Series CISM courses and lectures, vol. 555 .

55. E.J. Doedel, R. Paffenroth, A.R. Champneys, T.F. Fairgrieve, Y.A. Kuznetsov, B.E. Oldeman, B. Sandstede, and $\mathrm{X}$. Wang. Auto 2000: Continuation and bifurcation software for ordinary differential equations. Technical report, Concordia University, 2002.

56. J. J. Thomsen. Vibrations and stability. Advanced theory, analysis and tools. Springer, 2003. 2nd edition.

57. H. Poincaré. Les méthodes nouvelles de la mécanique céleste. Gauthiers-Villars, Paris, 1892.

58. J. Guckenheimer and P. Holmes. Nonlinear oscillations, dynamical systems and bifurcations of vector fields. SpringerVerlag, New-York, 1983.

59. L. Jézéquel and C. H. Lamarque. Analysis of non-linear dynamical systems by the normal form theory. Journal of Sound and Vibration, 149(3):429-459, 1991.

60. S. A. Neild, A. R. Champneys, D. J. Wagg, T. L. Hill, and A. Cammarano. The use of normal forms for analysing nonlinear mechanical vibrations. Proc. R. Soc. A., 373:20140404, 2015

61. D. J. Wagg. Normal form transformations for structural dynamics: an introduction for linear and nonlinear systems. Journal of Structural Dynamics, 1.

62. A. Opreni, N. Boni, R. Carminati, and A. Frangi. Analysis of the nonlinear response of piezo-micromirrors with the harmonic balance method. Actuators, 10(2):21, 2021.

63. A. Dhooge, W. Govaerts, and Y. A. Kuznetsov. Matcont: a matlab package for numerical bifurcation analysis of odes. ACM Transactions on Mathematical Software (TOMS), 29(2):141-164, 2003. 
64. B. Cochelin and C. Vergez. A high order purely frequency-based harmonic balance formulation for continuation of periodic solutions. Journal of Sound and Vibration, 324(1):243 - 262, 2009.

65. R. Veltz. BifurcationKit.jl, July 2020.

66. M. Faraday. On a peculiar class of acoustical figures; and on certain forms assumed by groups of particles upon vibrating elastic surfaces. In Abstracts of the Papers Printed in the Philosophical Transactions of the Royal Society of London, number 3, pages 49-51. The Royal Society London, 1837.

67. É. Mathieu. Mémoire sur le mouvement vibratoire d'une membrane de forme elliptique. J. Math., 2(13):137-203, 1968.

68. A. H. Nayfeh and D. T. Mook. Nonlinear oscillations. John Wiley \& sons, New-York, 1979.

69. L. D. Zavodney and A. H. Nayfeh. The non-linear response of a slender beam carrying a lumped mass to a principal parametric excitation: theory and experiment. International journal of non-linear mechanics, 24(2):105-125, 1989.

70. W. Lacarbonara, H. Yabuno, and K. Hayashi. Non-linear cancellation of the parametric resonance in elastic beams: theory and experiment. International Journal of Solids and Structures, 44(7-8):2209-2224, 2007.

71. A. Givois, A. Grolet, O. Thomas, and J.-F. Deü. On the frequency response computation of geometrically nonlinear flat structures using reduced-order finite element models. Nonlinear Dynamics, 97(2):1747-1781, 2019.

72. C. Comi, A. Corigliano, V. Zega, and S. Zerbini. Non linear response and optimization of a new z-axis resonant micro-accelerometer. Mechatronics, 40:235-243, 2016.

73. V. Zega, A. Opreni, G. Mussi, H.-K. Kwon, G. Vukasin, G. Gattere, G. Langfelder, A. Frangi, and T. W. Kenny. Thermal stability of DETF MEMS resonators: numerical modelling and experimental validation. In 2020 IEEE 33rd International Conference on Micro Electro Mechanical Systems (MEMS), pages 1207-1210. IEEE, 2020.

74. S. Dou, B. S. Strachan, S. W. Shaw, and J. S. Jensen. Structural optimization for nonlinear dynamic response. Philosophical Transactions of the Royal Society A: Mathematical, Physical and Engineering Sciences, 373(2051):20140408, 2015.

75. S. Dou and J. S. Jensen. Optimization of nonlinear structural resonance using the incremental harmonic balance method. Journal of Sound and Vibration, 334:239-254, 2015.

76. D. A. Czaplewski, C. Chen, D. López, O. Shoshani, A. M. Eriksson, S. Strachan, and S. W. Shaw. Bifurcation generated mechanical frequency comb. Physical review letters, 121(24):244302, 2018.

77. C. Chen, D. H. Zanette, D. A. Czaplewski, S. W. Shaw, and D. López. Direct observation of coherent energy transfer in nonlinear micromechanical oscillators. Nature communications, 8(1):1-7, 2017.

\section{A Weak formulation of the invariance equations}

As remarked in Section 3 an alternative representation of the nonlinear change of coordinates is obtained by defining the nonlinear mappings between the normal coordinates and the continuous fields (here displacements and velocities), rather than operating from the nodal values from the FE discretisation. This alternative formulation will thus provide a more general setting, where one could for example use another numerical method for the space discretisation of the problem.

For the purpose of deriving this generalization, let us first restart from the finite element approximation, which expressed the involved fields as a linear combination of a finite set of basis functions:

$$
\boldsymbol{u}_{h}=\sum_{i=1}^{\mathrm{N}} \boldsymbol{l}_{i} \mathrm{U}_{i}, \quad \boldsymbol{w}_{h}=\sum_{i=1}^{\mathrm{N}} \boldsymbol{l}_{i} \mathrm{~W}_{i}
$$

with $l_{i}$ nodal shape functions. For the present treatment the same shape functions are used to interpolate both displacement and test fields. We can then introduce a coordinate change between normal coordinates and continous fields of the type:

$$
\boldsymbol{u}_{h}=\boldsymbol{\Psi}_{h}(\mathbf{z})
$$

with:

$$
\boldsymbol{\Psi}_{h}(\mathbf{z})=\sum_{p=0}^{o}\left[\boldsymbol{\Psi}_{h, \mathcal{I}}(\mathbf{z})\right]_{p}
$$

We can therefore expand each tensor order as:

$$
\left[\boldsymbol{\Psi}_{h}(\mathbf{z})\right]_{p}=\sum_{\mathcal{I}} \boldsymbol{\Psi}_{h, \mathcal{I}}^{(p)} \pi_{\mathcal{I}}^{(p)}
$$

within this setting, each mapping field $\boldsymbol{\Psi}_{h, \mathcal{I}}^{(p)}$ is a continuous field since it is defined pointwise over the entire domain B. We can then express each mapping field using a finite element approximation identical to that used for the displacement field itself:

$$
\boldsymbol{\Psi}_{h, \mathcal{I}}^{(p)}=\sum_{i=1}^{\mathrm{N}} \Psi_{h, \mathcal{I}, i}^{(p)} \boldsymbol{l}_{i}
$$

where $\Psi_{h, \mathcal{I}, i}^{(p)}$ corresponds to the nodal values of the mappings. We remark that these nodal values are mathematically equivalent to the entries of the mapping vectors used on the discretised problem presented in the work. This representation is exploited for the computation of the left-hand sides of the homological equations, without the need to compute the complete nonlinearity tensors, which is faster and more memory efficient. To better appreciate it, let us report the weak form associated to the first order autonomous problem: 


$$
\begin{aligned}
& \int_{\mathrm{B}} \rho \dot{\boldsymbol{v}}_{h} \cdot \boldsymbol{w}_{h} d \mathrm{~B}+\int_{\mathrm{B}} \varepsilon\left(\boldsymbol{u}_{h}\right): \mathcal{A}: \varepsilon\left(\boldsymbol{w}_{h}\right) d \mathrm{~B} \\
& +\int_{\mathrm{B}} \frac{1}{2} \gamma\left(\boldsymbol{u}_{h}, \boldsymbol{u}_{h}\right): \mathcal{A}: \varepsilon\left(\boldsymbol{w}_{h}\right)+\varepsilon\left(\boldsymbol{u}_{h}\right): \mathcal{A}: \gamma\left(\boldsymbol{u}_{h}, \boldsymbol{w}_{h}\right) d \mathrm{~B} \\
& +\int_{\mathrm{B}} \frac{1}{2} \gamma\left(\boldsymbol{u}_{h}, \boldsymbol{u}_{h}\right): \mathcal{A}: \gamma\left(\boldsymbol{u}_{h}, \boldsymbol{w}_{h}\right) d \mathrm{~B}=0 \quad \forall \boldsymbol{w}_{h} \in \mathcal{C}_{h}(\mathbf{0}) . \\
& \int_{\mathrm{B}} \rho \dot{\boldsymbol{u}}_{h} \cdot \boldsymbol{w}_{h} d \mathrm{~B}=\int_{\mathrm{B}} \rho \boldsymbol{v}_{h} \cdot \boldsymbol{w}_{h} \quad \forall \boldsymbol{w}_{h} d \mathrm{~B} \in \mathcal{C}_{h}(\mathbf{0}) .
\end{aligned}
$$

where the finite-dimensional velocity field $\dot{\boldsymbol{u}}_{h}=\boldsymbol{v}_{h}$ has been introduced. Time derivatives are taken exactly as for the discrete problem:

$$
\dot{\boldsymbol{u}}_{h}=\nabla_{\mathbf{z}} \boldsymbol{\Psi}_{h}(\mathbf{z}) \boldsymbol{f}(\mathbf{z}), \quad \dot{\boldsymbol{v}}_{h}=\nabla_{\mathbf{z}} \boldsymbol{\Upsilon}_{h}(\mathbf{z}) \boldsymbol{f}(\mathbf{z}),
$$

And upon substitution of mapping fields and their time derivatives into Eq. 133a we retrieve again the invariance equations, which are now formulated at the level of the weak form of the partial differential equation:

$$
\begin{aligned}
& \int_{\mathrm{B}} \rho \nabla_{\mathbf{z}} \boldsymbol{\Upsilon}_{h}(\mathbf{z}) \boldsymbol{f}(\mathbf{z}) \cdot \boldsymbol{w}_{h} d \mathrm{~B}+\int_{\mathrm{B}} \varepsilon\left(\boldsymbol{\Psi}_{h}(\mathbf{z})\right): \mathcal{A}: \boldsymbol{\varepsilon}\left(\boldsymbol{w}_{h}\right) d \mathrm{~B} \\
& +\int_{\mathrm{B}} \frac{1}{2} \gamma\left(\Psi_{h}(\mathbf{z}), \Psi_{h}(\mathbf{z})\right): \mathcal{A}: \boldsymbol{\varepsilon}\left(\boldsymbol{w}_{h}\right)+\boldsymbol{\varepsilon}\left(\boldsymbol{\Psi}_{h}(\mathbf{z})\right): \mathcal{A}: \gamma\left(\boldsymbol{\Psi}_{h}(\mathbf{z}), \boldsymbol{w}_{h}\right) d \mathrm{~B} \\
& +\int_{\mathrm{B}} \frac{1}{2} \gamma\left(\boldsymbol{\Psi}_{h}(\mathbf{z}), \boldsymbol{\Psi}_{h}(\mathbf{z})\right): \mathcal{A}: \gamma\left(\boldsymbol{\Psi}_{h}(\mathbf{z}), \boldsymbol{w}_{h}\right) d \mathrm{~B}=0 \quad \forall \boldsymbol{w}_{h} \in \mathcal{C}_{h}(\mathbf{0}) . \\
& \int_{\mathrm{B}} \rho \nabla_{\mathbf{z}} \boldsymbol{\Psi}_{h}(\mathbf{z}) \boldsymbol{f}(\mathbf{z}) \cdot \boldsymbol{w}_{h} d \mathrm{~B}=\int_{\mathrm{B}} \rho \boldsymbol{\Upsilon}_{h}(\mathbf{z}) \cdot \boldsymbol{w}_{h} d \mathrm{~B} \quad \forall \boldsymbol{w}_{h} \in \mathcal{C}_{h}(\mathbf{0}) .
\end{aligned}
$$

These two invariance equations are completely equivalent to Eq. 29, if the finite element approximation is identical and if the same integration rules are used. The interesting feature of Eq. 135a is that it represents, for each monomial of the asymptotic expansion, a linear elliptic partial differential equation, already discretised in a finite dimensional space. As a result, all matrices and left-hand sides can be computed from the integration of Eq. 135a without the need to export the full nonlinearity tensor of the system. Also, Eq. 135a can be used in a more general setting and one could enlarge the scope and select another numerical method than the finite element procedure to solve out the elliptic PDEs.

\section{B Multivariate tensor notation}

Most quantities adopted in the present work are coefficients of multivariate polynomials. In this Section we recall the tensorial representation introduced in 35 to derive such quantities.

A generic vector of multivariate polynomials $\mathbf{a}(\mathbf{z})$ can be expressed as a summation of terms of increasing order from zero, up to the maximum order of the expansion $o$ :

$$
\mathbf{a}(\mathbf{z})=\sum_{p=0}^{o}[\mathbf{a}(\mathbf{z})]_{p} .
$$

for each expansion order, polynomial terms are explicitly defined as:

$$
[\mathbf{a}(\mathbf{z})]_{p}=\sum_{i_{1}=1}^{n} \sum_{i_{2}=1}^{n} \ldots \sum_{i_{p}=1}^{n} \mathbf{a}_{\left\{i_{1} i_{2} \ldots i_{p}\right\}}^{(p)} \mathbf{z}_{i_{1}} \mathbf{z}_{i_{2}} \ldots \mathrm{z}_{i_{p}},
$$

with $n$ the number of variables of the polynomial expansion. Alternatively, the following expansion can be used:

$$
[\mathbf{a}(\mathbf{z})]_{p}=\sum_{\mathcal{I}} \mathbf{a}_{\mathcal{I}}^{(p)} \pi_{\mathcal{I}}^{(p)}
$$

where indexes and variables products are collected with the following notation:

$$
\mathcal{I}=\left\{i_{1} i_{2} \ldots i_{p}\right\}, \quad \pi_{\mathcal{I}}^{(p)}=\mathrm{z}_{i_{1}} \mathrm{z}_{i_{2}} \ldots \mathrm{z}_{i_{p}} .
$$

For a given polynomial with order $p$, there exist a finite number of indexes combinations $\mathcal{I}$ corresponding to the expansion monomials. Such combinations are collected in sets $\mathcal{H}^{(p)}$. as:

As a natural consequence of the above notation, vectors belonging to an asymptotic expansion are in general expressed

$$
\mathbf{a}_{\mathcal{I}}^{(p)}=\mathbf{a}_{\left\{i_{1} i_{2} \ldots i_{p}\right\}}^{(p)},
$$

or alternatively, instead of writing vectors using the compact bold-typed notation, it is possible to express vector components using the following notation:

$$
\mathrm{a}_{s \mathcal{I}}^{(p)}=\mathrm{a}_{s\left\{i_{1} i_{2} \ldots i_{p}\right\}}^{(p)},
$$


where the first subscript $s$ stands for the $s$-th vector entry. If multiple vectors are present for a given monomial and order, as for instance when multiple forcing eigenvalues are present, a further index may be needed. Expansion are in general expressed as:

$$
\begin{array}{r}
\mathbf{a}_{j, \mathcal{I}}^{(p)}=\mathbf{a}_{j,\left\{i_{1} i_{2} \ldots i_{p}\right\}}^{(p)}, \\
\mathrm{a}_{j, s \mathcal{I}}^{(p)}=\mathrm{a}_{j, s\left\{i_{1} i_{2} \ldots i_{p}\right\}}^{(p)},
\end{array}
$$

where index $j$ refers to the $j$-th forcing eigenvalue.

\section{Nonlinearity tensors computation}

An important remark on dynamical systems stemming from partial differential equations is that their discretised algebraic formulation is equivalent to their finite dimensional weak formulation up to numerical integration errors. This has already been underlined in Appendix A] where a finite-dimensional weak form for the invariance equation is derived. The natural result is that nonlinearities can be computed through integration over the domain. Let us consider the expressions reported in Eqs. 38d)-38e . The most efficient technique to compute such term is simply to derive them through numerical integration of their associated weak formulation. Regarding the quadratic operator, its assemblage is performed as:

$$
\begin{aligned}
\mathbf{G}_{\mathcal{I}}^{(p)}=\sum_{k=1}^{p-1} \boldsymbol{G}\left(\boldsymbol{\Psi}_{\left\{i_{1} \ldots i_{k}\right\}}^{(k)}, \boldsymbol{\Psi}_{\left\{i_{k+1} \ldots i_{p}\right\}}^{(p-k)}\right)= \\
\sum_{k=1}^{p-1} \frac{1}{2} \int_{\mathrm{B}} \gamma\left(\boldsymbol{\Psi}_{h,\left\{i_{1} \ldots i_{k}\right\}}^{(k)}, \boldsymbol{\Psi}_{h,\left\{i_{k+1} \ldots i_{p}\right\}}^{(p-k)}\right): \mathcal{A}: \boldsymbol{\varepsilon}\left(\boldsymbol{w}_{h}\right) \\
+\boldsymbol{\varepsilon}\left(\boldsymbol{\Psi}_{h,\left\{i_{1} \ldots i_{k}\right\}}^{(k)}\right): \mathcal{A}: \gamma\left(\boldsymbol{\Psi}_{h,\left\{i_{k+1} \ldots i_{p}\right\}}^{(p-k)}, \boldsymbol{w}_{h}\right) \\
+\boldsymbol{\varepsilon}\left(\boldsymbol{\Psi}_{h,\left\{i_{k+1} \ldots i_{p}\right\}}^{(p-k)}\right): \mathcal{A}: \boldsymbol{\gamma}\left(\boldsymbol{\Psi}_{h,\left\{i_{1} \ldots i_{k}\right\}}^{(k)}, \boldsymbol{w}_{h}\right) d \mathrm{~B},
\end{aligned}
$$

where the $\boldsymbol{G}(\mathbf{U}, \mathbf{U})$ operator has been recasted in symmetric form. Similar treatment is performed for the $\boldsymbol{H}(\mathbf{U}, \mathbf{U}, \mathbf{U})$ operator:

$$
\begin{aligned}
& \mathbf{H}_{\mathcal{I}}^{(p)}=\sum_{k=1}^{p-2} \sum_{l=1}^{p-k-1} \boldsymbol{H}\left(\boldsymbol{\Psi}_{\left\{i_{1} \ldots i_{k}\right\}}^{(k)}, \mathbf{\Psi}_{\left\{i_{k+1} \ldots i_{k+l}\right\}}^{(l)}, \mathbf{\Psi}_{\left\{i_{k+l+1} \ldots i_{p}\right\}}^{(p-k-l)}\right)= \\
& \sum_{k=1}^{p-2} \sum_{l=1}^{p-k-1} \frac{1}{6} \int_{\mathrm{B}} \gamma\left(\boldsymbol{\Psi}_{h,\left\{i_{1} \ldots i_{k}\right\}}^{(k)}, \mathbf{\Psi}_{h,\left\{i_{k+1} \ldots i_{k+l}\right\}}^{(l)}\right): \mathcal{A}: \boldsymbol{\gamma}\left(\boldsymbol{\Psi}_{h,\left\{i_{k+l+1} \ldots i_{p}\right\}}^{(p-k-l)}, \boldsymbol{w}_{h}\right) \\
& +\gamma\left(\Psi_{h,\left\{i_{k+l+1} \ldots i_{p}\right\}}^{(p-k-l)}, \mathbf{\Psi}_{h,\left\{i_{1} \ldots i_{k}\right\}}^{(k)}\right): \mathcal{A}: \gamma\left(\Psi_{h,\left\{i_{k+1} \ldots i_{k+l}\right\}}^{(l)}, \boldsymbol{w}_{h}\right) \\
& +\gamma\left(\boldsymbol{\Psi}_{h,\left\{i_{k+1} \ldots i_{k+l}\right\}}^{(l)}, \boldsymbol{\Psi}_{h,\left\{i_{k+l+1} \ldots i_{p}\right\}}^{(p-k-l)}\right): \mathcal{A}: \boldsymbol{\gamma}\left(\boldsymbol{\Psi}_{h,\left\{i_{1} \ldots i_{k}\right\}}^{(k)}, \boldsymbol{w}_{h}\right) d \mathrm{~B},
\end{aligned}
$$

\section{Non-autonomous gradients}

In Eq. 32, terms involving a gradient with respect to the normal coordinate require a dedicated treatment. In particular, two new terms depending on such gradient are involved. The first involves the gradient of the non-autonomous mappings:

$$
\begin{aligned}
& \left.\left[\nabla_{\mathbf{z}} \hat{\boldsymbol{\Psi}}(\mathbf{z}) \boldsymbol{f}(\mathbf{z})\right]_{p}=\left[\sum_{s=1}^{2 \mathrm{n}}\left(\frac{\partial[\hat{\boldsymbol{\Psi}}(\mathbf{z})]_{\gtrless}{ }_{<p}}{\partial \mathbf{z}_{s}}+\frac{\partial[\hat{\boldsymbol{\Psi}}(\mathbf{z})]_{p}}{\partial \mathbf{z}_{s}}\right)\left(\lambda_{s} \mathbf{z}_{s}+\left[f_{s}(\mathbf{z})\right]_{\gtrless p}\right]\right)\right]_{p}, \\
& \left.\left[\nabla_{\mathbf{z}} \hat{\boldsymbol{\Upsilon}}(\mathbf{z}) \boldsymbol{f}(\mathbf{z})\right]_{p}=\left[\sum_{s=1}^{2 \mathrm{n}}\left(\frac{\partial[\hat{\boldsymbol{\Upsilon}}(\mathbf{z})]_{>1}<_{p}}{\partial \mathbf{z}_{s}}+\frac{\partial[\hat{\boldsymbol{r}}(\mathbf{z})]_{p}}{\partial \mathbf{z}_{s}}\right)\left(\lambda_{s} \mathbf{z}_{s}+\left[f_{s}(\mathbf{z})\right]_{\gtrless p}\right]\right)\right]_{p},
\end{aligned}
$$

where the shortcut notation $[\cdot]_{\gtrless} \underset{p}{1}$ has been introduced to refer to a term with order strictly larger than one and smaller than $p$. Expanding the product in the right-hand side, one can rewrite:

$$
\begin{aligned}
{\left[\nabla_{\mathbf{z}} \hat{\boldsymbol{\Psi}}(\mathbf{z}) \boldsymbol{f}(\mathbf{z})\right]_{p} } & =\sum_{s=1}^{2 \mathrm{n}}\left(\frac{\partial[\hat{\boldsymbol{\Psi}}(\mathbf{z})]_{p}}{\partial \mathbf{z}_{s}} \lambda_{s} \mathbf{z}_{s}+\left[\frac{\partial[\hat{\boldsymbol{\Psi}}(\mathbf{z})]_{\gtrless p}}{\partial \mathbf{z}_{s}}\left[f_{s}(\mathbf{z})\right]_{\gtrless p}\right]\right), \\
{\left[\nabla_{\mathbf{z}} \hat{\boldsymbol{Y}}(\mathbf{z}) \boldsymbol{f}(\mathbf{z})\right]_{p} } & =\sum_{s=1}^{2 \mathrm{n}}\left(\frac{\partial[\hat{\boldsymbol{\Upsilon}}(\mathbf{z})]_{p}}{\partial \mathbf{z}_{s}} \lambda_{s} \mathbf{z}_{s}+\left[\frac{\partial[\hat{\boldsymbol{\Upsilon}}(\mathbf{z})]_{\gtrless p}}{\partial \mathbf{z}_{s}}\left[f_{s}(\mathbf{z})\right]_{\gtrless p}\right]\right) .
\end{aligned}
$$


The second term at the right-hand side of Eq. 146 can be explicitly rewritten as:

$$
\begin{aligned}
& \left.\sum_{s=1}^{2 \mathrm{n}}\left[\frac{\partial[\hat{\boldsymbol{\Psi}}(\mathbf{z})]_{\gtrless p}{ }_{\gtrless p}}{\partial \mathrm{z}_{s}}\left[f_{s}(\mathbf{z})\right]_{\gtrless p}\right]_{p}\right]_{p}=\sum_{s=1}^{2 \mathrm{n}} \sum_{k=2}^{p} \frac{\partial[\hat{\boldsymbol{\Psi}}(\mathbf{z})]_{p-k+1}}{\partial \mathrm{z}_{s}}\left[f_{s}(\mathbf{z})\right]_{k}=\sum_{\mathcal{I}}\left(\overline{\boldsymbol{\mu}}_{\mathcal{I}}^{(p)}\right) \pi_{\mathcal{I}}^{(p)}, \\
& \sum_{s=1}^{2 \mathrm{n}}\left[\frac{\partial[\hat{\boldsymbol{r}}(\mathbf{z})]_{\gtrless 1}}{\partial \mathrm{z}_{s}}\left[f_{s}(\mathbf{z})\right]_{\gtrless p}\right]_{p}=\sum_{s=1}^{2 \mathrm{n}} \sum_{k=2}^{p} \frac{\partial[\hat{\boldsymbol{\Upsilon}}(\mathbf{z})]_{p-k+1}}{\partial \mathrm{z}_{s}}\left[f_{s}(\mathbf{z})\right]_{k}=\sum_{\mathcal{I}}\left(\overline{\boldsymbol{\nu}}_{\mathcal{I}}^{(p)}\right) \pi_{\mathcal{I}}^{(p)},
\end{aligned}
$$

where all the terms have been grouped and expanded onto the monomials $\pi_{\mathcal{I}}^{(p)}$, thus making appear new coefficients $\overline{\boldsymbol{\mu}}_{\mathcal{I}}^{(p)}$ and $\overline{\boldsymbol{\nu}}_{\mathcal{I}}^{(p)}$, the full expressions of which can be written as:

$$
\begin{aligned}
& \overline{\boldsymbol{\mu}}_{\mathcal{I}}^{(p)}=\sum_{s=1}^{2 \mathrm{n}} \sum_{k=2}^{p} \sum_{l=0}^{p-k} \hat{\boldsymbol{\Psi}}_{\left\{i_{1} \ldots i_{l} s i_{l+k+1} \ldots i_{p}\right\}}^{(p-k+1)} f_{s\left\{i_{l+1} \ldots i_{l+k}\right\}}^{(k)}, \\
& \overline{\boldsymbol{\nu}}_{\mathcal{I}}^{(p)}=\sum_{s=1}^{2 \mathrm{n}} \sum_{k=2}^{p} \sum_{l=0}^{p-k} \hat{\boldsymbol{\Upsilon}}_{\left\{i_{1} \ldots i_{l} s i_{l+k+1} \ldots i_{p}\right\}}^{(p-k+1)} f_{s\left\{i_{l+1} \ldots i_{l+k}\right\}}^{(k)} .
\end{aligned}
$$

These new terms have exactly the same structure as those appearing in Eqs. $38 \mathrm{~b}-3 \mathrm{38c}$ computed for the autonomous part, where one just need to replace the terms of the nonlinear mapping, which has important consequence at the computational level and ease the writing.

The second term involving a gradient and needing a dedicated development involves the gradient of the known autonomous mappings with respect to the normal coordinates, now multiplied with the reduced dynamics of the unknown time-dependent part. It can be expressed as:

$$
\begin{aligned}
& {\left[\nabla_{\mathbf{z}} \boldsymbol{\Psi}(\mathbf{z}) \hat{\boldsymbol{f}}(\mathbf{z})\right]=\left[\sum_{s=1}^{2 \mathrm{n}}\left(\boldsymbol{\phi}_{s}+\frac{\partial[\boldsymbol{\Psi}(\mathbf{z})]_{>1} \underset{\leq p}{ }}{\partial \mathbf{z}_{s}}\right)\left(\left[\hat{f}_{s}(\mathbf{z})\right]_{\gtrless p p}+\left[\hat{f}_{s}(\mathbf{z})\right]_{p}\right)\right]_{p},} \\
& {\left[\nabla_{\mathbf{z}} \boldsymbol{\Upsilon}(\mathbf{z}) \hat{\boldsymbol{f}}(\mathbf{z})\right]=\left[\sum_{s=1}^{2 \mathrm{n}}\left(\boldsymbol{\phi}_{s} \lambda_{s}+\frac{\partial[\boldsymbol{\Upsilon}(\mathbf{z})]_{\underset{\geq}{\leq p}}}{\partial \mathrm{z}_{s}}\right)\left(\left[\hat{f}_{s}(\mathbf{z})\right]_{\underset{<}{\gtrless 0}, 0}+\left[\hat{f}_{s}(\mathbf{z})\right]_{p}\right)\right]_{p} .}
\end{aligned}
$$

which in turn can be rewritten as:

$$
\begin{aligned}
& \left.\left[\nabla_{\mathbf{z}} \boldsymbol{\Psi}(\mathbf{z}) \hat{\boldsymbol{f}}(\mathbf{z})\right]=\sum_{s=1}^{2 \mathrm{n}}\left(\boldsymbol{\phi}_{s} \lambda_{s}\left[\hat{f}_{s}(\mathbf{z})\right]_{p}+\left[\frac{\partial[\boldsymbol{\Psi}(\mathbf{z})]_{\underset{\geq}{\geq} p}}{\partial \mathrm{z}_{s}}\left[\hat{f}_{s}(\mathbf{z})\right]_{\gtrless 0}\right]_{p}\right]_{p}\right),
\end{aligned}
$$

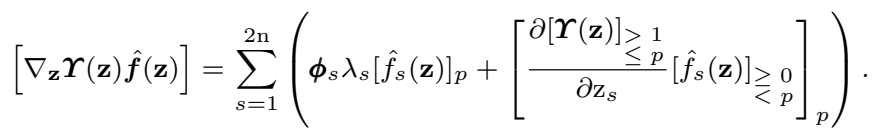

The second term on the right-hand side can be explicitly defined as:

$$
\begin{aligned}
& \left.\sum_{s=1}^{2 \mathrm{n}}\left[\frac{\left.\partial[\boldsymbol{\Psi}(\mathbf{z})]_{>1}\right]_{p}}{\partial \mathbf{z}_{s}}\left[\hat{f}_{s}(\mathbf{z})\right]_{\gtrless 0}\right]_{p}\right]_{p}=\sum_{s=1}^{2 \mathrm{n}} \sum_{k=0}^{p-1} \frac{\partial[\boldsymbol{\Psi}(\mathbf{z})]_{p-k+1}}{\partial \mathbf{z}_{s}}\left[\hat{f}_{\mathcal{s}}(\mathbf{z})\right]=\sum_{\mathcal{I}} \overline{\overline{\boldsymbol{\mu}}}_{\mathcal{I}}^{(p)} \pi_{\mathcal{I}}^{(p)},
\end{aligned}
$$

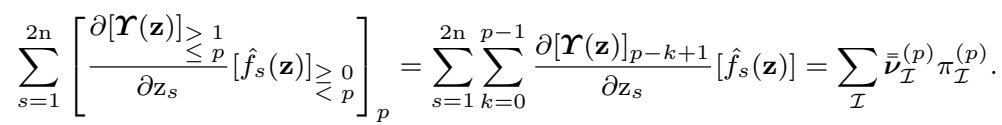

which provides the following compact expression for Eq. 150:

$$
\begin{aligned}
& {\left[\nabla_{\mathbf{z}} \boldsymbol{\Psi}(\mathbf{z}) \hat{\boldsymbol{f}}(\mathbf{z})\right]=\sum_{\mathcal{I}}\left(\sum_{s=1}^{2 \mathrm{n}}\left(\boldsymbol{\phi}_{s} \hat{f}_{s \mathcal{I}}^{(p)}\right)+\overline{\overline{\boldsymbol{\mu}}}_{\mathcal{I}}^{(p)}\right) \pi_{\mathcal{I}}^{(p)},} \\
& {\left[\nabla_{\mathbf{z}} \boldsymbol{\Upsilon}(\mathbf{z}) \hat{\boldsymbol{f}}(\mathbf{z})\right]=\sum_{\mathcal{I}}\left(\sum_{s=1}^{2 \mathrm{n}}\left(\boldsymbol{\phi}_{s} \lambda_{s} \hat{f}_{s \mathcal{I}}^{(p)}\right)+\overline{\bar{\nu}}_{\mathcal{I}}^{(p)}\right) \pi_{\mathcal{I}}^{(p)},}
\end{aligned}
$$

where the new terms provided by the above developments are explicitly computed as:

$$
\begin{aligned}
& \overline{\overline{\boldsymbol{\mu}}}_{\mathcal{I}}^{(p)}=\sum_{s=1}^{2 \mathrm{n}} \sum_{k=0}^{p-1} \sum_{l=0}^{p-k} \boldsymbol{\Psi}_{\left\{i_{1} \ldots i_{l} s i_{l+k+1} \ldots i_{p}\right\}}^{(p-k+1)} \hat{f}_{s\left\{i_{l+1} \ldots i_{l+k}\right\}}^{(k)}, \\
& \overline{\overline{\boldsymbol{\nu}}}_{\mathcal{I}}^{(p)}=\sum_{s=1}^{2 \mathrm{n}} \sum_{k=0}^{p-1} \sum_{l=0}^{p-k} \boldsymbol{\Upsilon}_{\left\{i_{1} \ldots i_{l} s i_{l+k+1} \ldots i_{p}\right\}}^{(p-k+1)} \hat{f}_{s\left\{i_{l+1} \ldots i_{l+k}\right\}}^{(k)} .
\end{aligned}
$$

Again, these coefficients can be directly compared to those reported in Eqs. 38b- $38 \mathrm{c}$ ) and in Eqs. 148, since sharing exactly the same structure, where now the non-autonomous part (tilde terms) is on the reduced dynamics coefficients. At the computational level, this symmetric structure can be used to compute easily these new terms. 


\section{E Explicit expressions for the RHS of low order $\varepsilon^{1}$-homological equations and reduced dynamics coefficients}

In the present Section we report explicit expressions of the right-hand sides $\hat{\mathbf{E}}_{\mathcal{I}}^{(p)}$ for low order developments. This serves the purpose of highlighting how different terms in the reduced dynamics are obtained and how they are affected by the forcing and the geometrical parameters of the structure.

For the zero-order homological equations, $\hat{\mathbf{E}}^{(0)}$ is simply given by the applied forcing:

$$
\begin{aligned}
\hat{\mathbf{E}}^{(0)} & =\hat{\mathbf{F}}, \\
\hat{\boldsymbol{\mu}}^{(0)} & =\mathbf{0} .
\end{aligned}
$$

Furthermore, since $\hat{\boldsymbol{\mu}}^{(0)}=\mathbf{0}$, the resulting mapping is co-linear with the applied forcing $\hat{\mathbf{F}}$ and reduced dynamics coefficients $\hat{\mathbf{F}}^{0}$ are proportional to the projection of the forcing vector onto the master modal subspace, as detailed in Section 4 This conclusion is obtained by considering that the reduced dynamics coefficient can be computed from the relation:

$$
\hat{f}_{j \mathcal{I}}^{(p)}=\mathbf{X}_{j}^{\mathrm{T}}\left[\begin{array}{c}
\hat{\mathbf{E}}_{\mathcal{I}}^{(p)} \\
-\mathbf{M} \hat{\boldsymbol{\mu}}_{\mathcal{I}}^{(p)}
\end{array}\right]=\frac{\boldsymbol{\phi}_{j}^{\mathrm{T}}}{\lambda_{j}-\bar{\lambda}_{j}} \hat{\mathbf{E}}_{\mathcal{I}}^{(p)}+\frac{\bar{\lambda}_{j} \boldsymbol{\phi}_{j}^{\mathrm{T}}}{\lambda_{j}-\bar{\lambda}_{j}} \mathbf{M} \hat{\boldsymbol{\mu}}_{\mathcal{I}}^{(p)}
$$

which for the zero-order development yields:

$$
\hat{f}_{j}^{(0)}=\frac{\boldsymbol{\phi}_{j}^{\mathrm{T}}}{\lambda_{j}-\bar{\lambda}_{j}} \hat{\mathbf{F}}
$$

The first-order non-autonomous asymptotic development yields:

$$
\begin{aligned}
\hat{\mathbf{E}}_{\mathcal{I}}^{(1)}=\hat{\mathbf{E}}_{\left\{i_{1}\right\}}^{(1)} & =-\mathbf{M} \sum_{s=1}^{2 \mathrm{n}}\left[\hat{\boldsymbol{\Upsilon}}_{\left\{s i_{1}\right\}}^{(2)} \hat{f}_{s}^{(0)}+\hat{\boldsymbol{\Upsilon}}_{\left\{i_{1} s\right\}}^{(2)} \hat{f}_{s}^{(0)}\right]-2 \boldsymbol{G}\left(\mathbf{\Psi}_{\left\{i_{1}\right\}}^{(1)}, \hat{\mathbf{\Psi}}^{(0)}\right), \\
\hat{\boldsymbol{\mu}}_{\left\{i_{1}\right\}}^{(1)} & =\sum_{s=1}^{2 \mathrm{n}}\left[\hat{\mathbf{\Psi}}_{\left\{s i_{1}\right\}}^{(2)} \hat{f}_{s}^{(0)}+\hat{\mathbf{\Psi}}_{\left\{i_{1} s\right\}}^{(2)} \hat{f}_{s}^{(0)}\right]
\end{aligned}
$$

thus providing an explicit expression for the reduced dynamics coefficients:

$\hat{f}_{j\left\{i_{1}\right\}}^{(1)}=\frac{\boldsymbol{\phi}_{j}^{\mathrm{T}}}{\lambda_{j}-\bar{\lambda}_{j}}\left[-\mathbf{M} \sum_{s=1}^{2 \mathrm{n}}\left[\hat{\boldsymbol{\Upsilon}}_{\left\{s i_{1}\right\}}^{(2)} \hat{f}_{s}^{(0)}+\hat{\boldsymbol{\Upsilon}}_{\left\{i_{1} s\right\}}^{(2)} \hat{f}_{s}^{(0)}\right]-2 \boldsymbol{G}\left(\boldsymbol{\Psi}_{\left\{i_{1}\right\}}^{(1)}, \hat{\mathbf{\Psi}}^{(0)}\right)\right]+\frac{\bar{\lambda}_{j} \boldsymbol{\phi}_{j}^{\mathrm{T}}}{\lambda_{j}-\bar{\lambda}_{j}}\left[\sum_{s=1}^{2 \mathrm{n}} \mathbf{M}\left[\hat{\boldsymbol{\Psi}}_{\left\{s i_{1}\right\}}^{(2)} \hat{f}_{s}^{(0)}+\hat{\mathbf{\Psi}}_{\left\{i_{1} s\right\}}^{(2)} \hat{f}_{s}^{(0)}\right]\right]$

which highlights how the reduced dynamics coefficients at first-order are heavily affected by the $\boldsymbol{G}\left(\boldsymbol{\Psi}_{i_{1}}^{(1)}, \hat{\boldsymbol{\Psi}}^{(0)}\right)$ vector. Finally, the second-order expansions yields:

$$
\begin{aligned}
\hat{\mathbf{E}}_{\mathcal{I}}^{(2)}=\hat{\mathbf{E}}_{\left\{i_{1} i_{2}\right\}}^{(2)}= & -\mathbf{M} \sum_{s=1}^{2 \mathrm{n}}\left[\boldsymbol{\Upsilon}_{\left\{s i_{1} i_{2}\right\}}^{(3)} \hat{f}_{s}^{(0)}+\boldsymbol{\Upsilon}_{\left\{i_{1} s i_{2}\right\}}^{(3)} \hat{f}_{s}^{(0)}+\boldsymbol{\Upsilon}_{\left\{i_{1} i_{2} s\right\}}^{(3)} \hat{f}_{s}^{(0)}\right] \\
& -\mathbf{M} \sum_{s=1}^{2 \mathrm{n}}\left[\boldsymbol{\Upsilon}_{\left\{i_{1} s\right\}}^{(2)} \hat{f}_{s\left\{i_{2}\right\}}^{(1)}+\boldsymbol{\Upsilon}_{\left\{s i_{2}\right\}}^{(2)} \hat{f}_{s\left\{i_{1}\right\}}^{(1)}\right]-\mathbf{M} \sum_{s=1}^{2 \mathrm{n}}\left[\hat{\boldsymbol{\Upsilon}}_{\{s\}}^{(1)} f_{s\left\{i_{1} i_{2}\right\}}^{(2)}\right] \\
& +2 \boldsymbol{G}\left(\boldsymbol{\Psi}_{\left\{i_{1} i_{2}\right\}}^{(2)}, \hat{\boldsymbol{\Psi}}^{(0)}\right)+2 \boldsymbol{G}\left(\boldsymbol{\Psi}_{\left\{i_{1}\right\}}^{(1)}, \hat{\mathbf{\Psi}}_{\left\{i_{2}\right\}}^{(1)}\right)+3 \boldsymbol{H}\left(\boldsymbol{\Psi}_{\left\{i_{1}\right\}}^{(1)}, \mathbf{\Psi}_{\left\{i_{2}\right\}}^{(1)}, \hat{\boldsymbol{\Psi}}^{(0)}\right), \\
\hat{\boldsymbol{\mu}}_{\left\{i_{1} i_{2}\right\}}^{(2)}= & -\sum_{s=1}^{2 \mathrm{n}}\left[\boldsymbol{\Psi}_{\left\{s i_{1} i_{2}\right\}}^{(3)} \hat{f}_{s}^{(0)}+\boldsymbol{\Psi}_{\left\{i_{1} s i_{2}\right\}}^{(3)} \hat{f}_{s}^{(0)}+\boldsymbol{\Psi}_{\left\{i_{1} i_{2} s\right\}}^{(3)} \hat{f}_{s}^{(0)}\right] \\
& -\sum_{s=1}^{2 \mathrm{n}}\left[\boldsymbol{\Psi}_{\left\{i_{1} s\right\}}^{(2)} \hat{f}_{s\left\{i_{2}\right\}}^{(1)}+\boldsymbol{\Psi}_{\left\{s i_{2}\right\}}^{(2)} \hat{f}_{s\left\{i_{1}\right\}}^{(1)}\right]-\sum_{s=1}^{2 \mathrm{n}}\left[\hat{\mathbf{\Psi}}_{\{s\}}^{(1)} f_{s\left\{i_{1} i_{2}\right\}}^{(2)}\right],
\end{aligned}
$$

and the second-order reduced dynamics coefficients:

$$
\begin{aligned}
\hat{f}_{j\left\{i_{1} i_{2}\right\}}^{(2)}=\frac{\boldsymbol{\phi}_{j}^{\mathrm{T}}}{\lambda_{j}-\bar{\lambda}_{j}}[ & -\mathbf{M} \sum_{s=1}^{2 \mathrm{n}}\left[\mathbf{\Upsilon}_{\left\{s i_{1} i_{2}\right\}}^{(3)} \hat{f}_{s}^{(0)}+\boldsymbol{\Upsilon}_{\left\{i_{1} s i_{2}\right\}}^{(3)} \hat{f}_{s}^{(0)}+\boldsymbol{\Upsilon}_{\left\{i_{1} i_{2} s\right\}}^{(3)} \hat{f}_{s}^{(0)}\right] \\
& -\mathbf{M} \sum_{s=1}^{2 \mathrm{n}}\left[\boldsymbol{\Upsilon}_{\left\{i_{1} s\right\}}^{(2)} \hat{f}_{s\left\{i_{2}\right\}}^{(1)}+\boldsymbol{\Upsilon}_{\left\{s i_{2}\right\}}^{(2)} \hat{f}_{s\left\{i_{1}\right\}}^{(1)}\right]-\mathbf{M} \sum_{s=1}^{2 \mathrm{n}}\left[\hat{\boldsymbol{\Upsilon}}_{\{s\}}^{(1)} f_{s\left\{i_{1} i_{2}\right\}}^{(2)}\right] \\
& \left.+2 \boldsymbol{G}\left(\boldsymbol{\Psi}_{\left\{i_{1} i_{2}\right\}}^{(2)}, \hat{\boldsymbol{\Psi}}^{(0)}\right)+2 \boldsymbol{G}\left(\boldsymbol{\Psi}_{\left\{i_{1}\right\}}^{(1)}, \hat{\boldsymbol{\Psi}}_{\left\{i_{2}\right\}}^{(1)}\right)+3 \boldsymbol{H}\left(\boldsymbol{\Psi}_{\left\{i_{1}\right\}}^{(1)}, \boldsymbol{\Psi}_{\left\{i_{2}\right\}}^{(1)}, \hat{\mathbf{\Psi}}^{(0)}\right)\right] \\
+\frac{\bar{\lambda}_{j} \boldsymbol{\phi}_{j}^{\mathrm{T}}}{\lambda_{j}-\bar{\lambda}_{j}}[ & -\mathbf{M} \sum_{s=1}^{2 \mathrm{n}}\left[\mathbf{\Psi}_{\left\{s i_{1} i_{2}\right\}}^{(3)} \hat{f}_{s}^{(0)}+\boldsymbol{\Psi}_{\left\{i_{1} s i_{2}\right\}}^{(3)} \hat{f}_{s}^{(0)}+\boldsymbol{\Psi}_{\left\{i_{1} i_{2} s\right\}}^{(3)} \hat{f}_{s}^{(0)}\right] \\
& \left.-\sum_{s=1}^{2 \mathrm{n}} \mathbf{M}\left[\mathbf{\Psi}_{\left\{i_{1} s\right\}}^{(2)} \hat{f}_{s i_{2}}^{(1)}+\boldsymbol{\Psi}_{\left\{s i_{2}\right\}}^{(2)} \hat{f}_{s\left\{i_{1}\right\}}^{(1)}\right]-\sum_{s=1}^{2 \mathrm{n}} \mathbf{M}\left[\hat{\mathbf{\Psi}}_{\{s\}}^{(1)} f_{s\left\{i_{1} i_{2}\right\}}^{(2)}\right]\right] .
\end{aligned}
$$

